Pacific

Journal of

Mathematics

INTÉGRABILITÉ LOCALE DES CARACTÈRES DE $\mathrm{SL}_{n}(D)$

BERTRAND LEMAIRE

Volume 222 No. 1

November 2005 


\title{
INTÉGRABILITÉ LOCALE DES CARACTÈRES DE $\mathrm{SL}_{n}(D)$
}

\author{
BERTRAND LEMAIRE
}

\begin{abstract}
Let $F$ be a nonarchimedean locally compact field, $G$ be the multiplicative group of a finite dimensional central simple $F$-algebra, and $G^{\prime}$ be the kernel of the reduced norm $\operatorname{det}^{\prime}: G \rightarrow F^{\times}$. We prove in this paper that for all distinguished open subgroup $H \subset G$ and all irreducible (smooth complex) representation $\pi$ of $H$, the character $\Theta_{\pi}=\operatorname{trace}(\pi)$ is a locally integrable distribution on $\boldsymbol{H}$, locally constant on the set of regular elements of $\boldsymbol{H}$. Then we deduce that for all irreducible representation $\pi^{\prime}$ of $G^{\prime}$, the character $\Theta_{\pi^{\prime}}=\operatorname{trace}\left(\pi^{\prime}\right)$ is a locally integrable distribution on $G^{\prime}$, locally constant on the set of regular elements of $G^{\prime}$.
\end{abstract}

\section{Introduction}

Soient $F$ un corps commutatif localement compact non archimédien, $D$ une algèbre à division de centre $F$, et $V$ un espace vectoriel à droite sur $D$. Le degré de $D$ sur $F$ et la dimension de $V$ sur $D$ sont supposés finis. On pose $A=\operatorname{End}_{D}(V)$ et $G=\operatorname{Aut}_{D}(V)$. Soit $G^{\prime}$ le noyau de la norme réduite $\operatorname{det}^{\prime}: G \rightarrow F^{\times}$. Rappelons que le choix d'une $D$-base de $V$ induit un isomorphisme de groupes $G^{\prime} \simeq \mathrm{SL}_{n}(D)$, $n=\operatorname{dim}_{D}(V)$. Un élément $g \in G$ est dit régulier si son polynôme caractéristique réduit est produit de polynômes irréductibles sur $F$, deux à deux distincts (on ne demande pas que ces derniers soient séparables sur $F$ ). On note $G_{\mathrm{r}}$ l'ensemble des éléments réguliers de $G$, et pour tout sous-groupe $J \subset G$ contenant $G^{\prime}$, on pose $J_{\mathrm{r}}=J \cap G_{\mathrm{r}}$.

Soit $\left(\pi^{\prime}, W\right)$ une représentation complexe lisse irréductible de $G^{\prime}$, et soit $d g^{\prime}$ une mesure de Haar sur $G^{\prime}$. On montre ici que le caractère $\Theta_{\pi^{\prime}}=\operatorname{trace}\left(\pi^{\prime} d g^{\prime}\right)$ défini par $\left\langle\phi, \Theta_{\pi^{\prime}}\right\rangle=\operatorname{trace}\left(\pi^{\prime}\left(\phi d g^{\prime}\right)\right)\left(\phi \in C_{\mathrm{c}}^{\infty}\left(G^{\prime}\right)\right)$ avec $\pi^{\prime}\left(\phi d g^{\prime}\right)=\int_{G^{\prime}} \phi\left(g^{\prime}\right) \pi^{\prime}\left(g^{\prime}\right) d g^{\prime}$, est une distribution localement intégrable sur $G^{\prime}$, localement constante sur $G_{\mathrm{r}}^{\prime}$. En d'autres termes, on montre qu'il existe une fonction $\lambda_{\pi^{\prime}}: G^{\prime} \rightarrow \mathbb{C}$ localement intégrable par rapport à $d g^{\prime}$ et localement constante sur $G_{\mathrm{r}}^{\prime}$, telle que $\Theta_{\pi^{\prime}}=\lambda_{\pi^{\prime}} d g^{\prime}$. La fonction $\left.\lambda_{\pi^{\prime}}\right|_{G_{\mathrm{r}}^{\prime}}$ est alors indépendante du choix de $d g^{\prime}$; on la note encore $\Theta_{\pi^{\prime}}$. Rappelons que pour $F$ de caractéristique nulle, ce résultat est vrai pour tout groupe

MSC2000: 22E50.

Mots-clefs: local field, central simple algebra, reduced norm, smooth complex representation, character of $\mathrm{SL}_{n}(D)$, twisted character of $\mathrm{GL}_{n}(D)$, Fourier transform, local integrability. 
$\boldsymbol{G}(F)$ où $\boldsymbol{G}$ est un groupe algébrique linéaire réductif défini sur $F$, connexe ou non [Harish-Chandra 1978; Clozel 1987]. Notons que pour $F$ de caractéristique $\geq 0$, on sait déjà [Harish-Chandra 1980] que $\Theta_{\pi^{\prime}}$ est une distribution localement constante sur l'ensemble des éléments semisimples réguliers (au sens habituel) de $G^{\prime}$. Pour $F$ de caractéristique $>0$ divisant $n$, l'intégrabilité locale des caractères de $G^{\prime}-$ conjecturée par Harish-Chandra - est un résultat nouveau : même pour $\mathrm{SL}_{2}(F)$ avec $F$ de caractéristique 2, il n'était pas connu jusqu'à présent.

Quelques mots sur l'intérêt d'un tel résultat. L'intégrabilité locale des caractères de $G^{\prime}$ est un outil fondamental pour l'analyse harmonique sur $G^{\prime}$. Il permet en particulier d'utiliser la formule d'intégration de Weyl, et d'attaquer certaines questions ouvertes en caractéristique $>0$ (e.g., l'orthogonalité des caractères des représentations de carré intégrable modulo le centre). D'autre part, si $\kappa$ est un caractère de $F^{\times}$trivial sur $\left(F^{\times}\right)^{n}$, le principe de la démonstration en deux étapes (cf. ci-dessous) permet de traiter les caractères tordus $\Theta_{\pi}^{\kappa}=\operatorname{trace}\left(\pi d g \circ A_{\pi}^{\kappa}\right)$ des représentations complexes lisses irréductibles $\kappa$-stables $\pi$ de $G$; où $A_{\pi}^{\kappa}$ désigne un opérateur d'entrelacement non nul entre $\pi \otimes\left(\kappa \circ \mathrm{det}^{\prime}\right)$ et $\pi$, et $d g$ une mesure de Haar sur $G$. On montre en particulier que ces caractères tordus sont des distributions localement intégrables sur $G$, localement constantes sur $G_{\mathrm{r}}$. Pour $F$ de caractéristique $>0$ et $D=F$, ce résultat a des applications importantes dans la théorie de l'induction automorphe : il permet par exemple, grâce au "lemme fondamental" [Henniart et Lemaire 2004b] et à l'existence de pseudo-coefficients pour le caractère tordu d'une série $\kappa$-discrète de $G$ [Henniart et Lemaire 2004a], de prouver par voie globale la surjectivité de l'application induction automorphe sans devoir utiliser les résultats d'Arthur - connus seulement en caractéristique nulle sur les caractères tempérés virtuels (voir [Henniart et Herb 1995]); la rédaction de tout cela apparaîtra dans un article ultérieur.

Passons maintenant à la description détaillée des résultats. Choisissons une uniformisante $\varpi$ de $F$. Identifions $F^{\times}$au centre $Z$ de $G$, et notons ${ }_{\varpi} G^{\prime}$ le sousgroupe fermé cocompact $\langle\varpi\rangle \cdot G^{\prime} \subset G$. Soit $(\tilde{\pi}, W)$ la représentation de ${ }_{\varpi} G^{\prime}$ définie par $\tilde{\pi}\left(\varpi^{i} g^{\prime}\right)=\pi^{\prime}\left(g^{\prime}\right)\left(i \in \mathbb{Z}, g^{\prime} \in G^{\prime}\right)$. Cette représentation s'étend en une représentation lisse $(\pi, W)$ d'un sous-groupe ouvert distingué d'indice fini $H \subset G$. Par construction, $\pi$ est admissible et irréductible. Soit $d h$ une mesure de Haar sur $H$. On est donc ramené à montrer que :

(1) le caractère $\Theta_{\pi}=\operatorname{trace}(\pi d h)$ défini par

$$
\left\langle f, \Theta_{\pi}\right\rangle=\operatorname{trace}(\pi(f d h)) \quad\left(f \in C_{\mathrm{c}}^{\infty}(H)\right)
$$

avec $\pi(f d h)=\int_{H} f(h) \pi(h) d h$, est une distribution localement intégrable sur $H$, localement constante sur $H_{\mathrm{r}}$; 
(2) si le point (1) est vérifié, alors $\Theta_{\pi^{\prime}}$ est une distribution localement intégrable sur $G^{\prime}$, localement constante sur $G_{\mathrm{r}}^{\prime}$; et l'on a $\Theta_{\pi^{\prime}}\left(g^{\prime}\right)=\Theta_{\pi}\left(g^{\prime}\right)$ pour tout $g^{\prime} \in G_{\mathrm{r}}^{\prime}$.

La démonstration des points (1) et (2) ci-dessus est l'objet principal de cet article.

Commençons par le point (1). Soit $H \subset G$ un sous-groupe ouvert distingué (il n'est pas nécessaire que $H$ soit d'indice fini). Il s'agit tout d'abord de généraliser la théorie de Howe [1974] : comme dans [Lemaire 2004], on montre que la transformée de Fourier $T^{\vee}$ d'une distribution $H$-invariante $T$ sur $A$ à support "suffisamment voisin du cône nilpotent" (voir la section 2 pour une définition précise), coïncide au voisinage de 0 dans $A$ avec la transformée de Fourier d'une distribution $H$-invariante sur $A$ à support compact modulo $H$-conjugaison; ce qui implique que $T^{\vee}$ est, au voisinage de 0 dans $A$, combinaison linéaire des transformées de Fourier des $H$-intégrales orbitales nilpotentes sur $A$. Rappelons que les $G$-orbites nilpotentes de $A$ sont paramétrées par l'ensemble $\Pi_{n}$ des partitions de $n$. Pour $\alpha \in \Pi_{n}$, la $G$-orbite nilpotente $\mathcal{O}_{\alpha}=\left\{g^{-1} n_{\alpha} g: g \in G\right\} \subset A$ associée à $\alpha$ est union disjointe finie de $H$-orbites, paramétrées par le groupe $\Delta_{H} \Delta_{\alpha} \backslash F^{\times}$; où l'on a posé $\Delta_{H}=\operatorname{det}^{\prime}(H)$ et $\Delta_{\alpha}=\operatorname{det}^{\prime}\left(G_{n_{\alpha}}\right)$. Pour $\alpha \in \Pi_{n}$ et $\bar{x} \in \Delta_{H} \Delta_{\alpha} \backslash F^{\times}$, la $H$-intégrale orbitale $\Theta_{\alpha, \bar{x}}$ définie par la $H$-orbite $\widehat{O}_{\alpha, \bar{x}}$ associée à $(\alpha, \bar{x})$, est donnée par une formule intégrale analogue à celle de Howe [1974, proposition 5] (proposition 1.5). La transformée de Fourier $\Theta_{\alpha, \bar{x}}^{\vee}$ de $\Theta_{\alpha, \bar{x}}$ est en revanche plus difficile à décrire; cf. (6.5) et la remarque A.6. Nous avons donc repris, en la modifiant pour l'adapter à la caractéristique $>0$, la partie 1 de [Harish-Chandra 1978] : on montre que pour toute distribution $H$-invariante $T$ sur $A$ à support compact modulo $H$-conjugaison, la transformée de Fourier $T^{\vee}$ est une distribution localement intégrable sur $A$, localement constante sur l'ouvert $A^{\prime} \subset A$ des éléments semisimples réguliers (au sens habituel); de plus, la fonction $\left.T^{\vee}\right|_{A^{\prime}}$ est donnée par une formule intégrale comme dans [Huntsinger 1997]. Notons que si $F$ est de caractéristique $>0$, ce dernier résultat est nouveau (i.e., n'avait pas encore été rédigé) même pour $D=F$ et $H=G$.

Soit $\pi$ une représentation complexe lisse irréductible (donc admissible) de $H$, et soit $x \in H$ un élément $H$-fermé; i.e., tel que la $H$-orbite de $x$ est fermée dans $H$ pour la topologie $\varpi$-adique. Alors une simple modification de la construction de [Lemaire 2004] (dans le cas non tordu) permet de "réduire" l'étude du caractère $\Theta_{\pi}$ au voisinage de $x$ dans $H$, à celle d'une distribution $H_{x}$-invariante $\theta \operatorname{sur} \operatorname{Lie}\left(H_{x}\right)=$ $\operatorname{Lie}\left(G_{x}\right)$ telle que le support de $\theta^{\vee}$ est "suffisamment voisin du cône nilpotent"; ici, $\theta^{\vee}$ désigne la transformée de Fourier de $\theta$ dans $\operatorname{Lie}\left(G_{x}\right)$. Puisque $\operatorname{Lie}\left(G_{x}\right)$ est une $F$-algèbre semisimple de la forme $B_{1} \times \cdots \times B_{r}$ où $B_{i}$ est une algèbre centrale simple sur une extension finie $F_{i}$ de $F$, on en déduit que la distribution $\Theta_{\pi}$ est intégrable (resp. constante si $x \in H_{\mathrm{r}}$ ) au voisinage de $x$ dans $H$; voir le 
corollaire 3.9 pour un énoncé précis (décomposition en germes). Puisque pour tout $y \in H$, la fermeture dans $H$ de la $H$-orbite de $y$ contient un élément $H$-fermé, le caractère $\Theta_{\pi}$ est localement intégrable sur tout le groupe $H$.

Quant au point (2), on se place dans la situation générale suivante : soit $H$ un groupe localement profini possédant une base dénombrable d'ouverts, et soient $G^{\prime}, C \subset H$ deux sous-groupes fermés distingués tels que :

- $G^{\prime} \cap C=\{1\}$,

- le groupe $C$ est central dans $H$ et discret (pour la topologie induite),

- le groupe $C G^{\prime} \backslash H$ est commutatif et compact (pour la topologie quotient).

On pose $\tilde{G}=C G^{\prime}$ (produit direct). On suppose les groupes $H$ et $G^{\prime}$ unimodulaires. Soit $\left(\pi^{\prime}, W\right)$ une représentation complexe lisse admissible irréductible de $G^{\prime}$. Notons $(\tilde{\pi}, W)$ la représentation de $\tilde{G}$ définie par $\tilde{\pi}(z g)=\pi^{\prime}\left(g^{\prime}\right)$ pour tous $z \in C$ et $g^{\prime} \in G^{\prime}$. On suppose que la représentation $(\tilde{\pi}, W)$ de $\tilde{G}$ s'étend en une représentation lisse $(\pi, W)$ de $H$. Puisque $\pi^{\prime}$ est admissible, $\pi$ l'est aussi. Soit $Z$ le centre de $H$, et soient $d h$ et $d g^{\prime}$ des mesures de Haar respectivement sur $H$ et $G^{\prime}$. On suppose que la distribution $\Theta_{\pi}=\operatorname{trace}(\pi d h)$ est localement intégrable sur $H$. On montre ici que si l'une des deux conditions suivantes est vérifiée :

(i) $\operatorname{vol}\left(G^{\prime} Z, d h\right) \neq \varnothing$ et la distribution $\Theta_{\pi}$ est localement constante sur une partie ouverte $\mathscr{y} \subset H$ telle que $\operatorname{vol}\left(G^{\prime} \backslash\left(\mathscr{y} \cap G^{\prime}\right), d g^{\prime}\right)=0$,

(ii) la projection canonique $H \rightarrow G^{\prime} \backslash H$ est scindée au-dessus d'un sous-groupe ouvert de $G^{\prime} \backslash H$,

alors la distribution $\Theta_{\pi^{\prime}}=\operatorname{trace}\left(\pi^{\prime} d g^{\prime}\right)$ est localement intégrable sur $G^{\prime}$. De plus, si la condition (i) est vérifiée, ou si la condition (ii) est vérifiée et si la distribution $\Theta_{\pi}$ est localement constante sur une partie ouverte $\mathscr{Y} \subset H$ telle que $\operatorname{vol}\left(G^{\prime} \backslash(\mathscr{Y} \cap\right.$ $\left.\left.G^{\prime}\right), d g^{\prime}\right)=0$, alors la fonction $\left.\Theta_{\pi}\right|_{\text {oyn }} G^{\prime}$ est localement intégrable sur $G^{\prime}$, et pour toute fonction $f^{\prime} \in C_{\mathrm{c}}^{\infty}\left(G^{\prime}\right)$, on a $\left\langle f^{\prime}, \Theta_{\pi^{\prime}}\right\rangle=\int_{G^{\prime}} f^{\prime}\left(g^{\prime}\right) \Theta_{\pi}(g) d g^{\prime}$. Dans cette égalité, $\Theta_{\pi}$ est une fonction localement constante sur $\mathscr{Y}$, indépendante du choix de $d h$ (voir plus haut).

Soit maintenant $\boldsymbol{G}$ un groupe algébrique linéaire réductif connexe défini sur $F$, et soit $\boldsymbol{G}^{\prime}$ son groupe dérivé. Soit $\boldsymbol{C}$ le tore central $F$-déployé maximal de $\boldsymbol{G}$, et soit $\boldsymbol{C}(\varpi)$ le sous-groupe de $\boldsymbol{C}(F)$ défini par $\boldsymbol{C}(\varpi)=\operatorname{Hom}\left(\mathrm{X}^{*}(\boldsymbol{C}),\langle\varpi\rangle\right)$. Posons ${ }_{\varpi} \boldsymbol{G}^{\prime}(F)=\boldsymbol{C}(\varpi) \boldsymbol{G}^{\prime}(F) \subset \boldsymbol{G}(F)$ (produit direct), et soit $H \subset \boldsymbol{G}(F)$ un sous-groupe ouvert contenant ${ }_{\varpi} \boldsymbol{G}^{\prime}(F)$. Le triplet $\left(H, \boldsymbol{G}^{\prime}(F), \boldsymbol{C}(\varpi)\right)$ vérifie toutes les conditions imposées au triplet $\left(H, G^{\prime}, C\right)$ dans le paragraphe précédent. Soit $\boldsymbol{G}(F)_{\mathrm{sr}}$ l'ensemble des éléments semisimples réguliers de $\boldsymbol{G}(F)$. Pour toute représentation complexe lisse irréductible (donc admissible) $\pi$ de $H$, on montre comme dans [Harish-Chandra 1980] que le caractère $\Theta_{\pi}$ est une fonction localement constante sur $H \cap \boldsymbol{G}(F)_{\text {sr }}$ (cet ensemble est ouvert et dense dans $H$ ). De plus, la projection canonique $\boldsymbol{G}(F) \rightarrow \boldsymbol{G}^{\prime}(F) \backslash \boldsymbol{G}(F)$ est scindée au-dessus de $\boldsymbol{G}^{\prime}(F) \backslash \boldsymbol{G}(F)$, par 
conséquent la condition (ii) est vérifiée (la condition (i) en revanche n'est pas toujours vérifiée). D'où le résultat cherché : pour toute représentation complexe lisse irréductible $\pi^{\prime}$ de $G^{\prime} \simeq \mathrm{SL}_{n}(D)$, le caractère $\Theta_{\pi^{\prime}}$ est une distribution localement intégrable sur $G^{\prime}$, localement constante sur $G_{\mathrm{r}}^{\prime}$. De plus, au voisinage d'un élément $G$-fermé $x$ de $G^{\prime}$, le caractère $\Theta_{\pi^{\prime}}$ possède une décomposition en germes, héritée de celle du caractère $\Theta_{\pi}$ d'une représentation lisse $\pi$ prolongeant $\pi^{\prime}$ à un sous-groupe ouvert d'indice fini de $G$. On montre aussi comment remplacer cette décomposition par une combinaison linéaire (finie) des transformées de Fourier des $G_{x}$-intégrales orbitales nilpotentes sur $\operatorname{Lie}\left(G_{x}\right)$ tordues par un caractère de $G_{x}$.

L'article est divisé en sept sections, suivies d'un appendice traitant des distributions $\left(\Theta_{\alpha}^{\kappa}\right)^{\vee}$. Ce sont :

1. Extension de la théorie de Howe [1974]

2. Transformées de Fourier des distributions $T \in J_{H}^{*}(A)$, d'après Harish-Chandra [1978]

3. Intégrabilité locale des caractères de $H$

4. Intégrabilité locale des caractères de $G^{\prime}$ : une méthode générale

5. Intégrabilité locale des caractères de $\boldsymbol{G}^{\prime}(F)$

6. Intégrabilité locale des caractères de $\mathrm{SL}_{n}(D)$

7. Application : intégrabilité locale des caractères tordus des représentations $\kappa$ stables de $\mathrm{GL}_{n}(D)$

Dans la section 2, on montre que la transformée de Fourier d'une distribution $H$ invariante sur $A$ à support compact, est localement constante sur $A^{\prime}$ et intégrable au voisinage de 0 dans $A$; l'intégrabilité locale sur $A$ est établie à la fin de la section 3. La section 3 reprend en la modifiant la construction de [Lemaire 2004] (dans le cas non tordu). La situation générale où $H$ est un groupe localement profini et $G^{\prime}, C \subset H$ sont deux sous-groupes fermés distingués, est traitée dans la section 4. Dans la section 5, on applique les résultats de la section 4 au cas particulier des groupes algébriques.

Conventions d'écriture, notations. Si $X$ est un espace topologique totalement discontinu. On note $C_{\mathrm{c}}^{\infty}(X)$ (resp. $C_{\mathrm{c}}(X)$ si $X$ discret, $C^{\infty}(X)$ si $X$ est compact, $C(X)$ si $X$ est fini) l'espace des fonctions $X \rightarrow \mathbb{C}$ localement constantes à support compact, et $\mathscr{D}(X)$ l'espace des distributions sur $X$. Si de plus $X$ est muni d'une structure de groupe topologique, on note $\epsilon(X)$ l'ensemble des classes d'équivalence de représentations complexes lisses irréductibles de $X$.

Une distribution $T$ sur un groupe topologique localement compact totalement discontinu $X$ est dite localement constante (resp. localement intégrable) sur une partie ouverte $Y \subset X$ s'il existe une mesure de Haar à gauche $d \mu$ sur $X$ et une 
fonction $\lambda: Y \rightarrow \mathbb{C}$ localement constante (resp. localement intégrable par rapport à $d \mu$ ) telle que $\left.T\right|_{Y}=\lambda d \mu$. À multiplication près par une constante $>0$, la fonction $\lambda$ ne dépend pas du choix de $d \mu$; lorsque la mesure de Haar sur $X$ est fixée (et qu'aucune autre ambiguïté n'est possible), on note $\lambda$ par la même lettre $T$.

Soient $X$ un groupe topologique localement compact totalement discontinu, et $\Omega \subset X$ une partie ouverte compacte. On appelle mesure de Haar sur $X$ normalisée par $\Omega$ l'unique mesure de Haar à gauche $d \mu$ sur $X$ telle que vol $(\Omega, d \mu)=1$. Plus généralement, pour tout sous-groupe fermé $Y \subset X$, on appelle mesure de Haar sur $Y$ normalisée par $\Omega$ l'unique mesure de Haar à gauche $d \mu^{\prime}$ sur $Y$ telle que $\operatorname{vol}\left(Y \cap \Omega, d \mu^{\prime}\right)=1$. Enfin si $X$ est compact, on appelle mesure de Haar normalisée sur $X$ la mesure de Haar sur $X$ normalisée par $X$.

En dehors de la section 4 (qui traite de groupes topologiques localement profinis), toutes les notions topologiques utilisées dans ce papier font référence à la topologie $\varpi$-adique; c'est pourquoi nous oublierons le plus souvent de le préciser.

\section{Extension de la théorie de Howe}

Soit $H$ un sous-groupe ouvert distingué de $G$ (un tel groupe contient $G^{\prime}$ ). On pose $\Delta_{H}=\operatorname{det}^{\prime}(H) \subset F^{\times}$.

Les classes de $G$-conjugaison dans $A$ sont décrites dans [Lemaire 2004, 5]. Reprenons le lexique introduit dans [Lemaire 2004, 6] : un élément $y \in A$ est dit

- fermé (ou $G$-fermé) si la $G$-orbite de $y$ est fermée dans $A$, i.e. si le polynôme minimal réduit de $y$ est produit de polynômes irréductibles sur $F$ deux à deux distincts ;

- pur (ou G-pur) si le polynôme minimal réduit de $y$ est irréductible sur $F$;

- régulier (ou G-régulier) si le polynôme caractéristique réduit de y est produit de polynômes irréductibles sur $F$ deux à deux distincts ;

- séparable (ou $G$-séparable) si chaque composante $F$-irréductible du polynôme minimal réduit de $y$ est séparable sur $F$.

Les éléments fermés séparables (resp. réguliers séparables) sont donc les éléments semisimples (resp. semisimples réguliers) au sens habituel. Un élément régulier est pur si et seulement si son centralisateur dans $G$ est compact modulo $Z$. Les éléments purs réguliers séparables sont donc les éléments semisimples réguliers elliptiques au sens habituel.

Soit $A_{\mathrm{r}}$ l'ensemble des éléments réguliers de $A$; on a donc $G_{\mathrm{r}}=G \cap A_{\mathrm{r}}$. Rappelons que $A^{\prime}$ désigne l'ensemble des éléments réguliers séparables de $A$ (et pas l'algèbre de Lie de $G^{\prime}$ !). Soit $A_{\mathrm{e}}^{\prime} \subset A^{\prime}$ le sous-ensemble formé des éléments purs.

Pour toutes parties $Y \subset A$ et $Q \subset G$, on note ${ }^{Q} Y=\operatorname{Ad} Q(Y)$ l'ensemble $\left\{g y g^{-1}\right.$ : $g \in Q, y \in Y\}$; si de plus $Y=\{y\}$, on pose $\mathscr{O}_{Q}(y)=Q_{\{y\}}$ et l'on note $Q_{y}$ le centralisateur $\left\{g \in Q: g y g^{-1}=y\right\}$ de $y$ dans $Q$. L'action par conjugaison de $G$ 
sur $A$ induit une action sur $\mathscr{D}(A)$ : pour $g \in G, T \in \mathscr{D}(A)$ et $f \in C_{\mathrm{c}}^{\infty}(A)$, on pose $\langle f, \operatorname{Ad} g(T)\rangle=\left\langle\operatorname{Ad}^{*} g^{-1}(f), T\right\rangle$ avec $\operatorname{Ad}^{*} g^{-1}(f)=f \circ \operatorname{Ad} g$.

Pour toute partie $X \subset A$, on note $\bar{X}$ la fermeture de $X$ dans $A$. Pour toute partie fermée $X \subset A$ telle que ${ }^{H} X=X$, on note $J_{H}(X)$ l'espace des distributions $H$ invariantes sur $X$ (pour l'action de $H$ par conjugaison) à support contenu dans $X$. Une partie $\Omega \subset A$ est dite compacte modulo $H$-conjugaison si elle est fermée et s'il existe une partie compacte $X \subset A$ telle que $\Omega \subset{ }^{H} X$. Puisque le groupe $Z H$ est cocompact (donc d'indice fini) dans $G, \Omega \subset A$ est une partie compacte modulo $H$-conjugaison si et seulement si elle est compacte modulo $G$-conjugaison. Soit $J_{H}^{*}=J_{H}^{*}(A) \subset J_{H}(A)$ le sous-espace formé des distributions à support compact modulo $H$-conjugaison.

Soient $\mathfrak{o}_{D}$ l'anneau des entiers de $D, \mathfrak{p}_{D}$ son idéal maximal, $d=(D: F)^{1 / 2}$, et $\omega$ la valuation sur $D$ normalisée par $\omega\left(D^{\times}\right)=\frac{1}{d} \mathbb{Z}$. Pour $y \in F$, on pose $|y|=q^{-\omega(y)}$ où $q$ désigne le cardinal du corps résiduel de $F$. Fixons un $\mathfrak{o}_{D}$-réseau $\Lambda$ dans $V$ et posons $\mathfrak{A}^{v}=\operatorname{Hom}_{\mathfrak{o}_{D}}\left(\Lambda, \Lambda \cdot \mathfrak{p}_{D}^{d v}\right)\left(v \in \frac{1}{d} \mathbb{Z}\right)$. Ainsi $\mathfrak{A}=\mathfrak{A}^{0}$ est un $\mathfrak{o}_{F}$-ordre maximal (donc héréditaire) dans $A$, de radical de Jacobson $\mathfrak{A}^{1 / d}$. Posons $K=\mathfrak{A}^{\times}$ et $K^{v}=1+\mathfrak{A}^{v}\left(v \in\left(\frac{1}{d} \mathbb{Z}\right)_{>0}\right)$.

Pour $v \in \frac{1}{d} \mathbb{Z}$, on note $j^{v}$ la projection canonique $\mathscr{D}(A) \rightarrow \mathscr{D}\left(A / \mathfrak{A}^{v}\right)$. Soit $\mathfrak{N}$ l'ensemble des éléments nilpotents de $A$, et pour $v \in \frac{1}{d} \mathbb{Z}$ et $X \subset A$, soit $J_{H, X}^{v}=$ $J_{H, X}^{v}(A)$ l'espace des distributions $H$-invariantes $T$ sur $A$ telles que pour $y \in A$, $\left\langle\mathbf{1}_{y+\mathfrak{A}^{v}}, T\right\rangle \neq 0$ implique que $y \in X$. On suppose dans cette section 1 que $n=$ $\operatorname{dim}_{D}(V)>1$.

Proposition 1.1. Il existe un voisinage ouvert et fermé $\mathscr{V}$ de $\mathfrak{N} \backslash\{0\}$ dans $A \backslash\{0\}$, tel que $F^{\times \mathscr{V}}=\mathscr{V}$ et $j^{v}\left(J_{H, \mathscr{V} \cup \mathfrak{A}^{\mu}}^{\nu}\right) \subset j^{\nu}\left(J_{H}^{*}\right)$ pour tous $v, \mu \in \frac{1}{d} \mathbb{Z}$.

Démonstration. Il s'agit simplement d'adapter la démonstration de [Lemaire 2004, 1.1]. Soit $n_{0} \in \mathfrak{N} \backslash\{0\}$. Comme en [Lemaire 2004, 1], on pose $V^{i}=\operatorname{ker} n_{0}^{i}(i=$ $0, \ldots, r ; n_{0}^{r-1} \neq 0$ et $n_{0}^{r}=0$ ). Soit $P$ le sous-groupe parabolique de $G$ défini par $P=\left\{g \in G: g\left(V^{i}\right)=V^{i}, i=1, \ldots, r-1\right\}$. Posons $W^{1}=V^{1}$, et pour $i=2, \ldots, r$, fixons un sous- $D$-espace vectoriel $W_{i}$ de $V_{i}$ tel que $V^{i} \cap \Lambda=\left(V^{i-1} \cap \Lambda\right) \oplus\left(W^{i} \cap \Lambda\right)$. Soit $M$ le sous-groupe de Lévi de $P$ défini par $M=\left\{g \in G: g\left(W^{i}\right)=W^{i}, i=\right.$ $1, \ldots, r\}$. On a la décomposition $M=\prod_{i=1}^{r} \operatorname{Aut}_{D}\left(W^{i}\right)$. Soit $Z(M)$ le centre de $M$, identifié à $\left(F^{\times}\right)^{r}$. L'élément $\delta \in Z(M)$ défini en [Lemaire 2004, 1] n'appartient pas nécessairement à $H$; on le remplace ici par l'élément $\delta^{\prime} \in Z(M) \cap G^{\prime}$ défini par

$$
\delta^{\prime}= \begin{cases}\left(\varpi^{-k}, \varpi^{-k+1}, \ldots, \varpi^{-1}, \varpi, \varpi^{2}, \ldots, \varpi^{k}\right) & \text { si } r=2 k, \\ \left(\varpi^{-k}, \varpi^{-k+1}, \ldots, \varpi^{-1}, 1, \varpi, \varpi^{2}, \ldots, \varpi^{k}\right) & \text { si } r=2 k+1 .\end{cases}
$$

Soient $U$ le radical unipotent de $P, \bar{U}$ le radical unipotent du sous-groupe parabolique $\bar{P}$ de $G$ opposé à $P$ par rapport à $M$, et $\mathfrak{u}, \overline{\mathfrak{u}} \subset A$ les sous- $F$-algèbres 
nilpotentes correspondantes. L'application $A \rightarrow A, g \mapsto \delta^{\prime} g \delta^{\prime-1}$ contracte $\mathfrak{u}$ et dilate $\overline{\mathfrak{u}}$. Précisément, pour $v \in \frac{1}{d} \mathbb{Z}$, on a les inclusions

$$
\begin{aligned}
& \mathfrak{u} \cap \mathfrak{A}^{v+n} \subset \delta^{\prime}\left(\mathfrak{u} \cap \mathfrak{A}^{v}\right) \delta^{\prime-1} \subset \mathfrak{u} \cap \mathfrak{A}^{v+1}, \\
& \overline{\mathfrak{u}} \cap \mathfrak{A}^{v-1} \subset \delta^{\prime}\left(\overline{\mathfrak{u}} \cap \mathfrak{A}^{v}\right) \delta^{\prime-1} \subset \overline{\mathfrak{u}} \cap \mathfrak{A}^{v-n} .
\end{aligned}
$$

Pour $v \in \frac{1}{d} \mathbb{Z}$, notons $\mathfrak{B}^{\prime v} l^{\prime} \mathfrak{o}_{F}$-réseau dans $A$ défini par $\mathfrak{B}^{\prime \nu}=\mathfrak{A}^{v} \cap \delta^{\prime} \mathfrak{A}^{v} \delta^{\prime-1}$. Le lemme 1.5 de [Lemaire 2004] reste vrai si l'on remplace $\mathfrak{B}^{v}$ par $\mathfrak{B}^{\prime \nu}$. Puisque $H$ est ouvert dans $G$, il existe un $\eta \in \frac{1}{d} \mathbb{Z}_{>0}$ tel que $K^{\eta} \subset H$. On en déduit que le lemme 1.6 de [Lemaire 2004] (condition (**) de [Howe 1974]) reste vrai si l'on remplace $G$ par $H$. La suite de la démonstration est identique celle de [Lemaire 2004, 1.1].

Soit $L / F$ une extension non ramifiée maximale contenue dans $D$, et soit $\varpi_{D}$ une uniformisante de $D$ telle que $\varpi_{D} L \varpi_{D}^{-1}=1$ et $\varpi_{D} y \varpi_{D}^{-1}=y^{\tau}(y \in L)$ pour un générateur $\tau$ de $\operatorname{Gal}(L / F)$. On peut supposer que $\varpi_{D}^{d}=\varpi$. On pose $A_{L}=A \otimes_{F} L$. Choisissons une $D$-base $\left(e_{1}, \ldots, e_{n}\right)$ de $V$. Soit $d=\operatorname{dim}_{F}(D)^{1 / 2}$. Les éléments $e_{i} \varpi_{D}^{d-j}(i=1, \ldots, n ; j=1, \ldots, d)$ forment une $L$-base de $V$. Pour $j=1, \ldots, d$, notons $V_{j}$ le sous- $L$-espace vectoriel de $V$ défini par $V_{j}=$ $\bigoplus_{i=1}^{n} e_{i} \varpi_{D}^{d-j} L$. Alors la $L$-décomposition $V=\bigoplus_{j=1}^{d} V_{j}$ induit une identification $A_{L}=\bigoplus_{1 \leq j, k \leq d} \operatorname{Hom}_{L}\left(V_{k}, V_{j}\right)$. Puisque pour $j=1, \ldots, d$, on a $\tau\left(V_{j}\right)=V_{j} \varpi_{D}^{-1}=$ $V_{j+1}$ où l'indice $j$ est considéré modulo $d \mathbb{Z}, A$ s'identifie à l'ensemble des matrices par blocs $\left(Y_{j, k}\right)_{1 \leq j, k \leq d} \in A_{L}, Y_{j, k} \in \operatorname{Hom}_{L}\left(V_{k}, V_{j}\right)$, telles que $\left(Y_{j, k}^{*}\right)^{\tau}=Y_{j+1, k+1}^{*}$ $(1 \leq j, k \leq d)$ où l'on a posé

$$
Y_{j, k}^{*}= \begin{cases}\varpi^{-1} Y_{j, k} & \text { si } j>k, \\ Y_{j, k} & \text { sinon. }\end{cases}
$$

D'après [Lemaire 2004, 5.3/1], les classes de $G$-conjugaison d'éléments nilpotents de $A$ sont paramétrées par l'ensemble $\Pi_{n}$ des partitions de $n$. On rappelle la construction. Soit $\alpha=\left(\alpha_{1} \geq \alpha_{2} \geq \cdots \geq \alpha_{n}\right) \in \Pi_{n}\left(\alpha_{i} \geq 0\right.$ et $\left.\sum_{i=1}^{n} \alpha_{i}=n\right)$. La $L$-base $\left(e_{1} \varpi_{D}^{d-1}, \ldots, e_{n} \varpi_{D}^{d-1}\right)$ de $V_{1}$ induit une identification $\operatorname{End}_{L}\left(V_{1}\right)=\mathrm{M}_{n}(L)$. Soit $\tilde{n}_{\alpha} \in \operatorname{End}_{L}\left(V_{1}\right)$ l'élément nilpotent défini par $\tilde{n}_{\alpha}=\operatorname{diag}\left(J_{\alpha_{1}}, \ldots, J_{\alpha_{r}}\right)$ (matrice diagonale par blocs) où $r$ est le plus grand entier $i \in\{1, \ldots, n\}$ tel que $\alpha_{i} \neq 0$, et

$$
J_{\alpha_{k}}=\left(\begin{array}{ccccc}
0 & 1 & 0 & \cdots & 0 \\
\vdots & \ddots & 1 & \ddots & \vdots \\
\vdots & & \ddots & \ddots & 0 \\
\vdots & & & \ddots & 1 \\
0 & \cdots & \cdots & \cdots & 0
\end{array}\right) \in M_{\alpha_{k}}(L)
$$


avec (convention d'écriture) $J_{1}=0$. Posons $n_{\alpha}=\bigoplus_{i=0}^{d-1} \tilde{n}_{\alpha}^{\tau^{i}} \in \mathfrak{N} \cap \bigoplus_{i=1}^{d} \operatorname{End}_{L}\left(V_{i}\right)$. L'application $\alpha \mapsto n_{\alpha}$ induit une bijection entre $\Pi_{n}$ et l'ensemble des classes de $G$-conjugaison d'éléments nilpotents de $A$, d'application réciproque la bijection induite par l'application $\mathfrak{N} \rightarrow \Pi_{n}, g \mapsto \phi^{-1}\left(\alpha_{g}\right)$ définie en [Lemaire 2004, 5].

Soit $\alpha \in \Pi_{n}$. Soient $\Delta_{\alpha}$ et $\Delta_{H, \alpha}$ les sous-groupes de $F^{\times}$définis par $\Delta_{\alpha}=$ $\operatorname{det}^{\prime}\left(G_{n_{\alpha}}\right)$ et $\Delta_{H, \alpha}=\operatorname{det}^{\prime}\left(H_{n_{\alpha}}\right)\left(=\Delta_{H} \cap \Delta_{\alpha}\right)$. Puisque $G^{\prime} \subset H$, l'application det $^{\prime}$ induit par restriction et passage aux quotients un isomorphisme de groupes $H_{n_{\alpha}} \backslash G_{n_{\alpha}} \rightarrow \Delta_{H, \alpha} \backslash \Delta_{\alpha}$. Le groupe $H G_{n_{\alpha}}$ est distingué dans $G$, et l'application $\operatorname{det}^{\prime}$ induit par passage aux quotients un isomorphisme de groupes $\delta_{\alpha}: H G_{n_{\alpha}} \backslash G \rightarrow$ $\Delta_{H} \Delta_{\alpha} \backslash F^{\times}$. Puisque $Z \subset G_{n_{\alpha}}$, le groupe $H G_{n_{\alpha}} \backslash G$ est fini. La $G$-orbite $\mathrm{O}_{\alpha}=\mathrm{O}_{G}\left(n_{\alpha}\right)$ est un $H$-ensemble (pour l'opération de $H$ par conjugaison), et via $\delta_{\alpha}$, l'ensemble des $H$-orbites de $\mathcal{O}_{\alpha}$ est un torseur sous $\Delta_{H} \Delta_{\alpha} \backslash F^{\times}$.

Remarque 1.2. Si l'on suppose seulement que $H$ est un sous-groupe distingué de $G$ contenant $G^{\prime}$, alors le groupe $\Delta_{H} \Delta_{\alpha} \backslash F^{\times}$peut être infini. En effet, pour $D=F$, $G=\mathrm{GL}_{2}(F)$ et $H=\mathrm{SL}_{2}(F)$, si $\alpha$ est la partition $(2,0)$, alors $n_{\alpha}$ est l'élément nilpotent $\left(\begin{array}{ll}0 & 1 \\ 0 & 0\end{array}\right)$ et $\Delta_{\alpha}=\left(F^{\times}\right)^{2}$. Or pour $F=\mathbb{F}_{2}((\varpi))$, le groupe $\left(F^{\times}\right)^{2} \backslash F^{\times}$est infini.

Soit $\alpha \in \Pi_{n}$. On pose $V_{\alpha}^{i}=\operatorname{ker} n_{\alpha}^{i}\left(i=0, \ldots, r_{\alpha} ; n_{\alpha}^{r_{\alpha}-1} \neq 0\right.$ et $\left.n_{\alpha}^{r_{\alpha}}=0\right)$. Soit $\mathfrak{p}_{\alpha}$ la sous- $F$-algèbre parabolique de $A$ associée à $n_{\alpha}$, définie par $\mathfrak{p}_{\alpha}=\{g \in A$ : $\left.g\left(V_{\alpha}^{i}\right) \subset V_{\alpha}^{i}, i=1, \ldots, r_{\alpha}-1\right\}$. Le radical nilpotent de $\mathfrak{p}_{\alpha}$ est la sous- $F$-algèbre nilpotente de $A$ définie par $\mathfrak{u}_{\alpha}=\left\{g \in A: g\left(V_{\alpha}^{i}\right) \subset V_{\alpha}^{i-1}, i=1, \ldots, r_{\alpha}-1\right\}$. On pose $P_{\alpha}=\mathfrak{p}_{\alpha} \cap G, P_{H, \alpha}=\mathfrak{p}_{\alpha} \cap H$ et $U_{\alpha}=\mathfrak{u}_{\alpha} \cap G$. D'après [Lemaire 2004, 1.4/1], on a l'inclusion $G_{n_{\alpha}} \subset P_{\alpha}$. Puisque $G^{\prime} \subset H$ et $G=G^{\prime} P_{\alpha}$, l'inclusion $P_{\alpha} \subset G$ induit par passage aux quotients un isomorphisme de groupes $\iota_{\alpha}: P_{H, \alpha} G_{n_{\alpha}} \backslash P_{\alpha} \rightarrow H G_{n_{\alpha}} \backslash G$. La $P_{\alpha}$-orbite $0_{\alpha}^{\bullet}=\mathrm{O}_{P_{\alpha}}\left(n_{\alpha}\right)$ est un $P_{H, \alpha}$-ensemble (pour l'opération de $P_{H, \alpha}$ par conjugaison); et via $\delta_{\alpha}^{\bullet}=\delta_{\alpha} \circ \iota_{\alpha}$, l'ensemble des $P_{H, \alpha}$-orbites de $\mathcal{O}_{\alpha}^{\bullet}$ muni du pointbase $\mathscr{O}_{P_{H, \alpha}}\left(n_{\alpha}\right)$, est un torseur sous $\Delta_{H} \Delta_{\alpha} \backslash F^{\times}$.

Soit $\Pi_{H, n}$ l'ensemble des couples $(\alpha, \bar{x})$ avec $\alpha \in \Pi_{n}$ et $\bar{x} \in \Delta_{H} \Delta_{\alpha} \backslash F^{\times}$. Pour $(\alpha, \bar{x}) \in \Pi_{H, n}$, choisissons un représentant $x$ de $\left(\delta_{\alpha}^{\bullet}\right)^{-1}(\bar{x})$ dans $P_{\alpha}$, et posons $n_{\alpha, x}=$ $x^{-1} n_{\alpha} x$; ainsi, $\mathfrak{p}_{\alpha}$ est la sous- $F$-algèbre parabolique de $A$ associée à $n_{\alpha, x}$. L'application $\Pi_{H, n} \rightarrow \mathfrak{N},(\alpha, \bar{x}) \mapsto n_{\alpha, x}$ induit une bijection entre $\Pi_{H, n}$ et l'ensemble des classes de $H$-conjugaison dans $\mathfrak{N}$. Pour $(\alpha, \bar{x}) \in \Pi_{H, n}$, on pose $\mathscr{O}_{\alpha, \bar{x}}=\mathscr{O}_{H}\left(n_{\alpha, x}\right)$ et $\mathrm{O}_{\alpha, \bar{x}}^{\bullet}=\mathfrak{O}_{P_{\alpha}}\left(n_{\alpha, x}\right)$; on a $\mathfrak{O}_{\alpha, \bar{x}}=x^{-1} \mathfrak{O}_{\alpha, 1} x$ et $\mathrm{O}_{\alpha, \bar{x}}^{\bullet}=x^{-1} \mathrm{O}_{\alpha, 1}^{\bullet} x$. Pour $\alpha \in \Pi_{n}$, on a donc $O_{\alpha}=\bigsqcup_{\bar{x}} O_{\alpha, \bar{x}}$ où $\bar{x}$ parcourt les éléments de $\Delta_{H} \Delta_{\alpha} \backslash F^{\times}$, et chaque $H$-orbite $\mathrm{O}_{\alpha, \bar{x}}$ est ouverte et fermée dans $\mathcal{O}_{\alpha}$. Pour $\alpha \in \Pi_{n}$, on a $\mathcal{O}_{\alpha}^{\bullet}=\mathfrak{O}_{\alpha} \cap \mathfrak{u}_{\alpha}$ [Lemaire 2004, 1.4/4], d'où $O_{\alpha, \bar{x}}^{\bullet}=\mathrm{O}_{\alpha, \bar{x}} \cap \mathfrak{u}_{\alpha}\left(\bar{x} \in \Delta_{H} \Delta_{\alpha} \backslash F^{\times}\right)$; de plus, on a $O_{\alpha}^{\bullet}=\bigsqcup_{\bar{x}} \mathrm{O}_{\alpha, \bar{x}}^{\bullet}$ où $\bar{x}$ parcourt les éléments de $\Delta_{H} \Delta_{\alpha} \backslash F^{\times}$, et chaque $P_{H, \alpha}$-orbite $\mathcal{O}_{\alpha, \bar{x}}^{\bullet}$ est ouverte et fermée dans $\mathrm{O}_{\alpha}^{\bullet}$. 
Lemme 1.3. Soit $\alpha \in \Pi_{n} \backslash\{(1, \ldots, 1)\}$. Pour $\bar{x} \in \Delta_{H} \Delta_{\alpha} \backslash F^{\times}, \mathscr{O}_{\alpha, \bar{x}}^{\bullet}$ est ouvert dans $\mathfrak{u}_{\alpha}$, et $\overline{\mathbb{O}_{\alpha, \bar{x}}^{\bullet}} \backslash \mathrm{O}_{\alpha, \bar{x}}^{\bullet}=\overline{\bar{O}_{\alpha, \bar{x}}^{\bullet}} \cap\left(\mathfrak{u}_{\alpha} \backslash \mathcal{O}_{\alpha}^{\bullet}\right)$.

Démonstration. Soit $\bar{x} \in \Delta_{H} \Delta_{\alpha} \backslash F^{\times}$. Puisque $\mathrm{O}_{\alpha}^{\bullet}$ est ouvert dans $\mathfrak{u}_{\alpha}$ [Lemaire 2004, 1.4/5] et $\mathrm{O}_{\alpha, \bar{x}}^{\bullet}$ est ouvert dans $\mathrm{O}_{\alpha}^{\bullet}, \mathrm{O}_{\alpha, \bar{x}}^{\bullet}$ est ouvert dans $\mathfrak{u}_{\alpha}$. Puisque $\mathrm{O}_{\alpha, \bar{x}}^{\bullet}$ est fermé dans $\mathcal{O}_{\alpha}^{\bullet}$, on a l'inclusion $\overline{\mathcal{O}_{\alpha, \bar{x}}^{\bullet}} \backslash \mathrm{O}_{\alpha, \bar{x}}^{\bullet} \subset \overline{\mathcal{O}_{\alpha}^{\bullet}} \backslash \mathrm{O}_{\alpha}^{\bullet}$. Or $\overline{\mathcal{O}_{\alpha}^{\bullet}}=\mathfrak{u}_{\alpha}$ [Lemaire 2004, 1.4/5], d'où l'inclusion $\overline{\mathcal{O}_{\alpha, \bar{x}}^{\bullet}} \backslash \mathrm{O}_{\alpha, \bar{x}}^{\bullet} \subset \overline{\mathrm{O}_{\alpha, \bar{x}}^{\bullet}} \cap\left(\mathfrak{u}_{\alpha} \backslash \mathrm{O}_{\alpha}^{\bullet}\right)$. L'inclusion inverse est claire.

Lemme 1.4. Pour tout sous-groupe $J \subset G$ contenant $G^{\prime}$ et tout sous-groupe parabolique $P \subset G$, on a la décomposition $J=(K \cap J)(P \cap J)$.

Démonstration. Soit $P \subset G$ un sous-groupe parabolique, que l'on peut supposer minimal. On a la décomposition d'Iwasawa $G=K P$. Soit $j \in J$. Écrivons $j=k p$ avec $k \in K$ et $p \in P$. Soit $x=\operatorname{det}^{\prime} k \in \mathfrak{o}_{F}^{\times}$, et soit $y \in \mathfrak{o}_{D}^{\times}$tel que $\operatorname{det}^{\prime} y=x$. Soit $\left(b_{1}, \ldots, b_{n}\right)$ une $D$-base de $V$ telle que $\Lambda=\bigoplus_{i=1}^{n} b_{i} \mathfrak{o}_{D}$. Cette base induit les identifications $K=\mathrm{GL}_{n}\left(\mathfrak{o}_{D}\right) \subset \mathrm{GL}_{n}(D)=G$. Soit $M \subset G$ le sous-groupe formé des matrices diagonales (à coefficients dans $D$ ), et soit $k_{0} \in K$ tel que $k_{0}^{-1} M k_{0} \subset P$. Alors $a=k_{0}^{-1} \operatorname{diag}(y, 1, \ldots, 1) k_{0} \in K \cap P$ et $\operatorname{det}^{\prime} a=x$. On a donc $j=\left(k a^{-1}\right)(a p)$ avec $k a^{-1} \in K \cap G^{\prime} \subset K \cap J$ et $a p=\left(k a^{-1}\right)^{-1} j \in P \cap J$. D'où le lemme.

Posons $K_{H}=K \cap H$, et soient $d g, d h$ et $d k$ les mesures de Haar respectivement sur $G, H$ et $K$ normalisées par $K_{H}$.

Puisque $H$ est ouvert dans $G$, pour $(\alpha, \bar{x}) \in \Pi_{n}$, le centralisateur $H_{n_{\alpha, x}}$ de $n_{\alpha, x}$ dans $H$ est ouvert dans $G_{n_{\alpha, x}}$, donc est unimodulaire [Lemaire 2004, 1.10/1]. Pour chaque $\alpha \in \Pi_{n}$, choisissons une mesure de Haar $d g_{n_{\alpha}}$ sur $G_{n_{\alpha}}$; notons $d h_{n_{\alpha}}$ la mesure de Haar sur $H_{n_{\alpha}}$ définie par $d h_{n_{\alpha}}=\left.d g_{n_{\alpha}}\right|_{H_{n_{\alpha}}}$; et pour $\bar{x} \in \Delta_{H} \Delta_{\alpha} \backslash F^{\times}$, notons $d h_{n_{\alpha, x}}$ la mesure de Haar sur $H_{n_{\alpha, x}}$ déduite de $d h_{n_{\alpha}}$ via l'isomorphisme $H_{n_{\alpha}} \rightarrow H_{n_{\alpha, x}}, h \mapsto x^{-1} h x$.

Proposition 1.5. Soit $\alpha \in \Pi_{n}$. Pour $\bar{x} \in \Delta_{H} \Delta_{\alpha} \backslash F^{\times}$, l'intégrale orbitale

$$
\Theta_{\alpha, \bar{x}}\left(f, d h_{n_{\alpha, x}}\right)=\int_{H_{n_{\alpha, x} \backslash H}} f\left(h^{-1} n_{\alpha, x} h\right) \frac{d h}{d h_{n_{\alpha, x}}} \quad\left(f \in C_{\mathrm{c}}^{\infty}(A)\right)
$$

est absolument convergente; i.e., $\Theta_{\alpha, \bar{x}}=\Theta_{\alpha, \bar{x}}\left(\cdot, d h_{n_{\alpha, x}}\right) \in J_{H}(A)$. De plus, fixée une mesure de Haar du sur $\mathfrak{u}_{\alpha}$, il existe une constante $c=c\left(d h_{n_{\alpha}}, d u\right)>0$ telle que pour tout $\bar{x} \in \Delta_{H} \Delta_{\alpha} \backslash F^{\times}$, on a

$$
\left\langle f, \Theta_{\alpha, \bar{x}}\right\rangle=c \iint_{K_{H} \times \overline{\mathbf{O}_{\alpha, \bar{x}}}} f\left(k^{-1} u k\right) d k d u \quad\left(f \in C_{\mathrm{c}}^{\infty}(A)\right) ;
$$

en particulier, la distribution $\Theta_{\alpha, \bar{x}}$ ne dépend pas du choix de $x \in P_{\alpha}$.

Démonstration. Si $\alpha=(1, \ldots, 1)$ (i.e., si $\left.n_{\alpha}=0\right)$, il n'y a rien à démontrer. On suppose donc que $\alpha \neq(1, \ldots, 1)$. Soit $d p$ la mesure de Haar à gauche sur $P_{\alpha}$ normalisée par $K_{H}$. Puisque $H=P_{H, \alpha} K_{H} 1.4$ et $P_{H, \alpha} \cap K_{H}=P_{\alpha} \cap K_{H}$, pour 
toute fonction intégrable $f: H \rightarrow \mathbb{C}$, on a $\int_{H} f(h) d h=\iint_{P_{H, \alpha} \times K_{H}} f(p k) d p d k$. Soit $\bar{x} \in \Delta_{H} \Delta_{\alpha} \backslash F^{\times}$, et soit $d y$ une mesure de Haar sur $H_{n_{\alpha, x}}$. Soit $d u$ une mesure de Haar sur $\mathfrak{u}_{\alpha}$. Grâce à 1.3, on montre comme dans [Laumon 1996, 4.8.9] (cf. aussi [Lemaire 2004, 3.2.2]) qu'il existe une constante $c(d y, d u)>0$ telle que pour toute fonction $f \in C_{\mathrm{c}}^{\infty}(A)$, on a

$$
\Theta_{\alpha, \bar{x}}(f, d y)=c(d y, d u) \iint_{K_{H} \times \overline{\sigma_{\alpha, \bar{x}}}} f\left(k^{-1} u k\right) d k d u .
$$

Ce qui implique que $\Theta_{\alpha, \bar{x}}(\cdot, d y) \in J_{H}(A)$. Soit $\delta_{P_{\alpha}}$ le caractère module sur $P_{\alpha}$, défini par $d\left(p^{\prime} p p^{\prime-1}\right)=\delta_{P_{\alpha}}(p) d p^{\prime}\left(p, p^{\prime} \in P_{\alpha}\right)$. Posons $K_{H}^{x}=x^{-1} K x$, et notons $d k^{\prime}$ la mesure de Haar normalisée sur $K_{H}^{x}$. En remplaçant $K$ par $K_{H}^{x}$ dans la construction précédente, on obtient de la même manière que pour toute fonction $f \in C_{\mathrm{c}}^{\infty}(A)$, on a

$$
\Theta_{\alpha, \bar{x}}(f, d y)=c(d y, d u) \delta_{P_{\alpha}}\left(x^{-1}\right) \iint_{K_{H}^{x} \times \overline{\dot{O}_{\alpha, \bar{x}}}} f\left(k^{\prime-1} u k^{\prime}\right) d k^{\prime} d u .
$$

Puisque $\Theta_{\alpha, \bar{x}}\left(\cdot, d h_{n_{\alpha, x}}\right)=\operatorname{Ad} x^{-1}\left(\Theta_{\alpha, 1}\left(\cdot, d h_{n_{\alpha}}\right)\right)$, on a

$$
c\left(d h_{n_{\alpha, x}}, d u\right)=c\left(d h_{n_{\alpha}}, d u\right) .
$$

Puisque chaque $G$-orbite nilpotente de $A$ est union finie de $H$-orbites, pour $(\alpha, \bar{x}) \in \Pi_{H, n}$, la $H$-orbite $\widehat{O}_{\alpha, \bar{x}}$ est localement fermée dans $A$; et $\overline{O_{\alpha, \bar{x}}}$ est réunion de $\mathrm{O}_{\alpha, \bar{x}}$ et d'un nombre fini de $H$-orbites nilpotentes de dimension (en tant que variétés $\varpi$-adiques) strictement inférieure à celle de $0_{\alpha, \bar{x}}$ [Lemaire 2004, 5.2/2]. On en déduit que les distributions $\Theta_{\alpha, \bar{x}}\left((\alpha, \bar{x}) \in \Pi_{H, n}\right)$ forment une base du $\mathbb{C}$ espace vectoriel $J_{H}(\mathfrak{N})$.

Soit $d_{A} g$ la mesure de Haar sur $A$ normalisée par $\mathfrak{A}$; pour tout $\mathfrak{o}_{F}$-ordre maximal $\mathfrak{Y}$ dans $A$, on a $\operatorname{vol}\left(\mathfrak{Y}, d_{A} g\right)=1$. Fixons un caractère $\Psi_{F}$ de $(F,+)$ de conducteur $\mathfrak{p}_{F}$ et posons $\Psi_{A}=\Psi_{F} \circ \operatorname{tr}_{A / F}^{\prime}$ où $\operatorname{tr}_{A / F}^{\prime}: A \rightarrow F$ désigne la trace réduite. On note $f \mapsto f^{\vee}$ la transformée de Fourier sur $C_{\mathrm{c}}^{\infty}(A)$ définie par $f^{\vee}(y)=$ $\int_{A} f(g) \overline{\Psi_{A}(y g)} d_{A} g$, où $z \mapsto \bar{z}$ désigne la conjugaison complexe. D'où une transformée de Fourier $T \mapsto T^{\vee}$ sur $\mathscr{D}(A)$, définie par $\left\langle f, T^{\vee}\right\rangle=\left\langle f^{\vee}, T\right\rangle\left(f \in C_{\mathrm{c}}^{\infty}(A)\right)$.

Proposition 1.6. Soit $\Omega \subset A$ une partie $H$-invariante compacte modulo $H$-conjugaison. Il existe un entier b tel que pour toute distribution $T \in J_{H}(\Omega)$, il existe des constantes $c_{\alpha, \bar{x}}(T)\left((\alpha, \bar{x}) \in \Pi_{H, n}\right)$ telles que

$$
\mathbf{1}_{\mathfrak{A}^{b}} \cdot\left(T^{\vee}-\sum_{(\alpha, \bar{x})} c_{\alpha, \bar{x}}(T) \Theta_{\alpha, \bar{x}}^{\vee}\right)=0
$$

où $(\alpha, \bar{x})$ parcourt les éléments de $\Pi_{H, n}$. 
Démonstration. Elle est identique à la démonstration de [Lemaire 2004, 1.11].

Pour $\alpha \in \Pi_{n}$, notons $\Theta_{\alpha}=\Theta_{\alpha}\left(\cdot, d g_{n_{\alpha}}\right) \in J_{G}(A)$ la distribution définie par $\left\langle f, \Theta_{\alpha}\right\rangle=\int_{G_{n_{\alpha}} \backslash G} f\left(g^{-1} n_{\alpha} g\right)\left(d g / d g_{n_{\alpha}}\right)$, où $f \in C_{\mathrm{c}}^{\infty}(A)$. D'après [Lemaire 2004, $1.10 / 3$ ], pour $\alpha \in \Pi_{n}$, la distribution $\Theta_{\alpha}^{\vee}$ est localement intégrable sur $A$, localement constante sur $A^{\prime}$. On va voir qu'il en est de même des distributions $\Theta_{\alpha, \bar{x}}^{\vee}$ pour $(\alpha, \bar{x}) \in \Pi_{H, n}$.

\section{Transformées de Fourier des distributions $T \in J_{H}^{*}(A)$, d'après Harish-Chandra}

Pour tout sous-groupe ouvert compact $\mathscr{K} \subset G$, on note $d_{\mathscr{K}} k$ la mesure de Haar normalisée sur $\mathscr{K}$, et l'on pose

$$
\bar{\Psi}_{A, y}^{\mathscr{K}}(g)=\int_{\mathscr{K}} \overline{\Psi_{A}\left(k y k^{-1} g\right)} d_{\mathscr{K}} k \quad(y, g \in A) .
$$

Proposition 2.1. Soit $T \in J_{H}^{*}(A)$. La transformée de Fourier $T^{\vee}$ est une distribution localement intégrable sur $A$, localement constante sur $A^{\prime}$. Et pour $y \in A^{\prime}$, on $a T^{\vee}(y)=\left\langle\bar{\Psi}_{A, y}^{\mathscr{K}}, T\right\rangle$ pour tout sous-groupe ouvert compact $\mathscr{K} \subset H$.

Pour $y \in A$, la fonction $A \rightarrow \mathbb{C}, g \mapsto \bar{\Psi}_{A, y}^{\mathscr{K}}(g)$ est clairement localement constante. Si de plus $y \in A^{\prime}$, on montrera en particulier que pour toute partie compacte modulo $H$-conjugaison $\Omega \subset A$, l'intersection $\operatorname{Supp}\left(\bar{\Psi}_{A, y}^{\mathscr{K}}\right) \cap \Omega$ est compacte ; ce qui donnera un sens à l'énoncé ci-dessus. L'idée sous-jacente à la proposition 2.1 est la suivante : soit $T \in J_{H}^{*}(A)$ et $f \in C_{\mathrm{c}}^{\infty}(A)$. On a

$$
\left\langle f, T^{\vee}\right\rangle=\int_{A}\left(\int_{A^{\prime}} f(y) \overline{\Psi_{A}(g y)} d_{A} y\right) d T(g),
$$

mais il n'est en général pas possible d'inverser les deux signes intégrales ci-dessus. Puisque $T$ est $H$-invariante, pour tout sous-groupe ouvert compact $\mathscr{K} \subset H$, on a aussi

$$
\left\langle f, T^{\vee}\right\rangle=\int_{A}\left(\int_{A^{\prime}} f(y) \bar{\Psi}_{A, y}^{\mathscr{K}}(g) d_{A} y\right) d T(g) .
$$

Il s'agit de montrer que les deux signes intégrales dans (2.2) peuvent s'inverser, et que la fonction $A \rightarrow \mathbb{C}$ définie presque partout par $y \mapsto \int_{A} \bar{\Psi}_{A, y}^{\mathscr{K}}(y) d T(y)$ est localement intégrable.

Dans cette section, nous ne démontrerons qu'une partie de la proposition 2.1, à savoir : l'intégrabilité de $T^{\vee}$ au voisinage de 0 dans $A$, la constance locale de $T^{\vee}$ sur $A^{\prime}$, et la formule pour $T^{\vee}(y)\left(y \in A^{\prime}\right)$. L'intégrabilité locale de $T^{\vee}$ sur $A$ sera montrée dans la section 3 (cf. le théorème 3.16). 


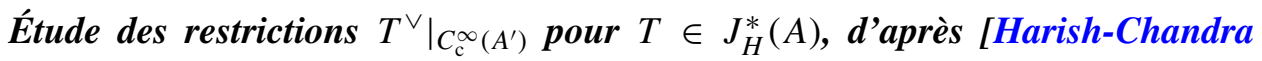
1978] et [Huntsinger 1997]. Fixons un sous-groupe ouvert compact $\mathscr{K} \subset H$. Soit $\Xi \subset{ }^{G}\left(\mathfrak{A}^{-1}\right)$ la partie $G$-invariante ouverte et fermée dans $A$ définie en [Lemaire 2004] 1.2. Pour tout sous-groupe de Cartan $\Gamma \subset G$, on pose $L(\Gamma)=\operatorname{Lie}(\Gamma) \subset A$ et $L(\Gamma)^{\prime}=L(\Gamma) \cap A^{\prime}$. Pour $x, y \in A$, on pose ad $x(y)=x y-y x$.

Lemme 2.3. Soient $y, g \in A$. Alors l'application $\mathscr{K} \rightarrow F, k \mapsto \operatorname{tr}_{A / F}^{\prime}\left(k y k^{-1} g\right)$ est submersive au point $k_{0} \in \mathscr{K}$ si et seulement si ad $g\left(k_{0} y k_{0}^{-1}\right) \neq 0$.

Démonstration. La différentielle de l'application $\mathscr{K} \rightarrow F, k \mapsto \operatorname{tr}_{A / F}^{\prime}\left(k y k^{-1} g\right)$ au point $k_{0}$ s'écrit :

$$
A \rightarrow F, x \mapsto \operatorname{tr}_{A / F}^{\prime}\left(\operatorname{ad} g\left(k_{0} y k_{0}^{-1}\right) x\right) .
$$

Par $F$-linéarité, cette différentielle est surjective si et seulement si elle est non identiquement nulle; i.e., si et seulement si ad $g\left(k_{0} y k_{0}^{-1}\right) \neq 0$. D'où le lemme.

Lemme 2.4. Soient $\Gamma \subset G$ un sous-groupe de Cartan et $\Omega \subset A$ une partie compacte modulo G-conjugaison. Alors l'intersection $L(\Gamma) \cap \Omega$ est compacte.

Démonstration. L'application $A \rightarrow F^{n d}, g \mapsto\left(a_{n d-1}(g), \ldots, a_{0}(g)\right)$ donnée par les coefficients du polynôme caractéristique réduit de $g$, est $G$-invariante; et sa restriction à $L(\Gamma)$ est propre, à fibres finies. D'où le lemme.

Proposition 2.5. Soient $k \in \mathbb{Z}$ et $\omega \subset A^{\prime}$ une partie ouverte compacte. Alors il existe un $a \in \mathbb{Z}$ tel que pour tout $y \in \omega$, on a $\operatorname{Supp}\left(\left.\bar{\Psi}_{A, y}\right|_{\varpi^{k}}\right) \subset \mathfrak{A}^{a}$.

Démonstration. Pour tout sous-groupe de Cartan $\Gamma \subset G$, l'application

$$
\mathscr{K} \times L(\Gamma)^{\prime} \rightarrow A^{\prime}, \quad(k, y) \mapsto k y k^{-1}
$$

est partout submersive; en particulier, l'intersection ${ }^{\mathscr{K}}\{L(\Gamma)\} \cap A^{\prime}$ est ouverte dans $A$. On en déduit qu'il existe un ensemble fini $\left\{\Gamma_{1}, \ldots, \Gamma_{s}\right\}$ de sous-groupes de Cartan de $G$ tel que $\omega \subset \bigcup_{i=1}^{s}{ }^{\mathscr{K}}\left\{L\left(\Gamma_{i}\right)\right\} \cap A^{\prime}$. Pour $i=1, \ldots, s$, l'intersection $L\left(\Gamma_{i}\right) \cap \varpi^{k} \Xi$ est compacte 2.4 , par conséquent $X_{i}={ }^{\mathscr{K}}\left\{L\left(\Gamma_{i}\right)\right\} \cap \varpi^{k} \Xi$ est une partie compacte. Soit $b \in \mathbb{Z}$ tel que $\bigcup_{i=0}^{s} X_{i} \subset \mathfrak{A}^{b}$, et posons $\Omega=\varpi^{k} \Xi \cap\left(A \backslash \mathfrak{A}^{b}\right)$. On a donc $\left(\bigcup_{i=1}^{s} \mathscr{K}_{\{}\left\{\left(\Gamma_{i}\right)\right\}\right) \cap \Omega=\varnothing$. Notons que $\Omega$ est une partie ouverte et fermée dans $A$, compacte modulo $G$-conjugaison. Puisque ${ }^{\mathscr{K}} \omega \subset \bigcup_{i=1}^{s}{ }^{\mathscr{K}}\left\{L\left(\Gamma_{i}\right)\right\}$, l'application

$$
\zeta: \mathscr{K} \times \omega \times \Omega \rightarrow F \times \omega \times \Omega,(k, y, g) \mapsto\left(\operatorname{tr}_{A / F}^{\prime}\left(k y k^{-1} g\right), y, g\right)
$$

est partout submersive 2.3. On peut donc lui appliquer le principe de submersion d'Harish-Chandra [1970, theorem 11] (voir [Harish-Chandra 1964, theorem 1] pour la démonstration) : il existe une unique application linéaire surjective

$$
C_{\mathrm{c}}^{\infty}(\mathscr{K} \times \omega \times \Omega) \rightarrow C_{\mathrm{c}}^{\infty}(\zeta(\mathscr{K} \times \omega \times \Omega)), \varphi \mapsto \varphi^{\zeta}
$$


telle que

$$
\begin{aligned}
& \iiint_{\mathscr{T} \times \omega \times \Omega} \varphi(k, y, g) \Phi \circ \zeta(k, y, g) d_{\mathscr{T}} k d_{A} y d_{A} g \\
& =\iiint_{F \times \omega \times \Omega} \varphi^{\zeta}(t, y, g) \Phi(t, y, g) d t d_{A} y d_{A} g
\end{aligned}
$$

pour toutes fonctions $\varphi \in C_{\mathrm{c}}^{\infty}(\mathscr{K} \times \omega \times \Omega)$ et $\Phi \in C_{\mathrm{c}}^{\infty}(\zeta(\mathscr{K} \times \omega \times \Omega))$; où $d t$ désigne la mesure de Haar sur $F$ normalisée par $\mathfrak{o}_{F}$. Soit $f_{0}=\left(\varphi_{0}\right)^{\zeta}$ avec $\varphi_{0}=\mathbf{1}_{\mathscr{K} \times \omega \times \Omega}$. Fixons un couple $(y, g) \in \omega \times \Omega$. Alors il existe un $c \in \mathbb{Z}$ tel que $y+\mathfrak{A}^{c} \subset \omega$, $g+\mathfrak{A}^{c} \subset \Omega$ et ${ }^{\mathscr{K}}\left(\mathfrak{A}^{c}\right) \mathfrak{A}^{c} \subset \mathfrak{A}^{1 / d}$ (rappelons que $\Psi_{A}\left(\mathfrak{A}^{1 / d}\right)=1$ ). Pour $\lambda \in F^{\times}$, notons $\bar{\Psi}_{F}^{\lambda}$ le caractère de $F$ défini par $\bar{\Psi}_{F}^{\lambda}(t)=\overline{\Psi_{F}(\lambda t)}$. Pour $\varphi=\varphi_{0}$ et $\Phi=$ $\left.\left(\bar{\Psi}_{F}^{\lambda} \otimes \mathbf{1}_{y+\mathfrak{A}^{c}} \otimes \mathbf{1}_{g+\mathfrak{A}^{c}}\right)\right|_{C_{\mathrm{c}}^{\infty}(\zeta(\mathscr{K} \times \omega \times \Omega))}$, on obtient

$$
\int_{\mathscr{K}} \overline{\Psi_{A}\left(\lambda k y k^{-1} g\right)} d_{\mathscr{K}} k=\int_{F} f_{0}(t, y, g) \bar{\Psi}_{F}^{\lambda}(t) d t .
$$

L'égalité ci-dessus est vraie pour tout $(y, g, \lambda) \in \omega \times \Omega \times F^{\times}$.

La fonction $f_{0}$ appartient à $C_{\mathrm{c}}^{\infty}(F \times \omega \times \Omega)=C_{\mathrm{c}}^{\infty}(F) \otimes C_{\mathrm{c}}^{\infty}(\omega) \otimes C_{\mathrm{c}}^{\infty}(\Omega)$. Par conséquent, il existe un $e \in \mathbb{Z}$ tel que pour tout $(y, g) \in \omega \times \Omega$, la fonction $t \mapsto f_{0}(t, y, g)$ appartient à $C_{\mathrm{c}}\left(F / \mathfrak{p}_{F}^{e}\right)$. Soit $\lambda_{0} \in F^{\times}$l'élément défini par

$$
\lambda_{0}= \begin{cases}1 & \text { si } e \leq 0, \\ \varpi^{-e} & \text { sinon. }\end{cases}
$$

Puisque le conducteur de $\bar{\Psi}_{F}^{\lambda_{0}}$ est contenu dans $\mathfrak{p}_{F}^{1+e}$, pour tout $(y, g) \in \omega \times \Omega$, on a $\int_{F} f_{0}(t, y, g) \bar{\Psi}_{F}^{\lambda}(t) d t=0$, d'où $\bar{\Psi}_{y}^{\mathscr{K}}\left(\lambda_{0} g\right)=0$. En d'autres termes, pour tout $(y, g) \in \omega \times \lambda_{0} \Omega$, on a $\bar{\Psi}_{y}^{\mathscr{K}}(g)=0$. Puisque $\mathfrak{o}_{F} \Xi=\Xi$ ([Lemaire 2004] 1.2), on a l'inclusion $\lambda_{0} \Omega \supset \varpi^{k} \Xi \cap\left(A \backslash \mathfrak{A}^{a}\right)$ avec $a=\inf \{b, b-e\}$. D'où la proposition.

Corollaire 2.6. Pour $y \in A^{\prime}$, la fonction $\left.\bar{\Psi}_{A, y}^{\Re}\right|_{\varpi^{k} \Xi}$ est à support compact. Et l'application $A^{\prime} \rightarrow C_{\mathrm{c}}^{\infty}\left(\varpi^{k} \Xi\right),\left.y \mapsto \bar{\Psi}_{A, y}^{\mathscr{K}}\right|_{\varpi^{k} \Xi}$ est localement constante.

Démonstration. La première assertion est claire. Quant à la seconde, soit $\omega \subset A^{\prime}$ une partie ouverte compacte, et choisissons un entier $a$ comme en 2.5. Il existe un entier $c \geq a$ tel que $\omega+\mathfrak{A}^{c}=\omega,\left(\varpi^{k} \Xi \cap \mathfrak{A}^{a}\right)+\mathfrak{A}^{c}=\varpi^{k} \Xi \cap \mathfrak{A}^{a}$ et ${ }^{\mathscr{K}}\left(\mathfrak{A}^{c}\right) \mathfrak{A}^{c} \subset$ $\mathfrak{A}^{1 / d}$. Alors pour tout $y \in \Omega$, on a $\left.\bar{\Psi}_{A, y}^{\mathscr{K}}\right|_{\varpi^{k} \Xi} \in C\left(\mathfrak{A}^{a} / \mathfrak{A}^{c}\right)$, et l'application $\omega \rightarrow$ $C_{\mathrm{c}}^{\infty}\left(\varpi^{k} \Xi\right),\left.y \mapsto \bar{\Psi}_{A, y}^{\mathscr{K}}\right|_{\varpi^{k} \Xi}$ se factorise à travers l'espace quotient $\omega / \mathfrak{A}^{c}$.

Proposition 2.7. Soit $T \in J_{H}^{*}(A)$. La transformée de Fourier $T^{\vee}$ est localement constante sur $A^{\prime}$. Et pour $y \in A^{\prime}$, on a $T^{\vee}(y)=\left\langle\bar{\Psi}_{A, y}^{\mathscr{K}}, T\right\rangle$.

Démonstration. Soient $f \in C_{\mathrm{c}}^{\infty}\left(A^{\prime}\right), \omega=\operatorname{Supp}(f)$ et $k \in \mathbb{Z}$ tel que $\operatorname{Supp}(T) \subset \varpi^{k} \Xi$. D'après 2.6, il existe un entier $a$ tel que $\operatorname{Supp}\left(\left.\bar{\Psi}_{A, y}^{\mathscr{K}}\right|_{\varpi^{k}} \Xi\right) \subset \mathfrak{A}^{a}$ pour tout $y \in \omega$. 
Et d'après (2.2), on a

$$
\left\langle f, T^{\vee}\right\rangle=\int_{\mathfrak{A}^{a}}\left(\int_{\omega} f(y) \bar{\Psi}_{A, y}^{\mathscr{K}}(g) d_{A} y\right) d T(g) .
$$

Puisque l'application $\omega \times \mathfrak{A}^{a} \rightarrow \mathbb{C},(y, g) \mapsto f(y) \bar{\Psi}_{A, y}^{\mathscr{K}}(g)$ est localement constante 2.6, on peut inverser les deux signes intégrales ci-dessus. On obtient que

$$
\begin{aligned}
\left\langle f, T^{\vee}\right\rangle & =\int_{\omega} f(y)\left\{\int_{\mathfrak{A}^{a}} \bar{\Psi}_{A, y}^{\mathscr{K}}(g) d T(g)\right\} d_{A} y \\
& =\int_{A} f(y)\left(\int_{A} \bar{\Psi}_{A, y}^{\mathscr{K}}(g) d T(g)\right) d_{A} y .
\end{aligned}
$$

À nouveau d'après 2.6, l'application $A^{\prime} \rightarrow\left\langle\bar{\Psi}_{A, y}^{\mathscr{K}}, T\right\rangle=\int_{A} \bar{\Psi}_{A, y}^{\mathscr{K}}(g) d T(g)$ est localement constante. D'où la proposition.

Descente parabolique. Posons $W^{1}=V_{(n)}^{1}$ avec (convention d'écriture)

$$
(n)=(n, 0, \ldots, 0) \in \Pi_{n},
$$

et pour $i=2, \ldots, n$, choisissons un sous- $D$-espace vectoriel $W^{i}$ de $V_{(n)}^{i}$ tel que $V_{(n)}^{i} \cap \Lambda=\left(V_{(n)}^{i-1} \cap \Lambda\right) \oplus\left(W^{i} \cap \Lambda\right)$. On a les décompositions $V=\bigoplus_{i=1}^{n} W^{i}$ et $\Lambda=\bigoplus_{i=1}^{n}\left(W^{i} \cap \Lambda\right)$. Soit $\mathfrak{m}_{(n)}$ la sous- $F$-algèbre de Lévi de $\mathfrak{p}_{(n)}$ définie par $\mathfrak{m}_{(n)}=\left\{g \in A: g\left(W^{i}\right) \subset W^{i}, i=1, \ldots, n\right\} ;$ si $D=F, \mathfrak{m}_{(n)}$ est une sous- $F$-algèbre de Cartan de $A$. Pour $\alpha \in \Pi_{n}$, notons $\mathfrak{m}_{\alpha}$ l'unique sous- $F$-algèbre de Lévi de $\mathfrak{p}_{\alpha}$ telle que $\mathfrak{m}_{\alpha} \supset \mathfrak{m}_{(n)}, \overline{\mathfrak{p}}_{\alpha}$ la sous- $F$-algèbre parabolique de $A$ opposée à $\mathfrak{p}_{\alpha}$ par rapport à $\mathfrak{m}_{\alpha}$, et $\overline{\mathfrak{u}}_{\alpha}$ le radical nilpotent de $\overline{\mathfrak{p}}_{\alpha}$. On a les décompositions : $A=\overline{\mathfrak{u}}_{\alpha} \oplus \mathfrak{m}_{\alpha} \oplus \mathfrak{u}_{\alpha}$ et $\mathfrak{A}=\left(\overline{\mathfrak{u}}_{\alpha} \cap \mathfrak{A}\right) \oplus\left(\mathfrak{m}_{\alpha} \cap \mathfrak{A}\right) \oplus\left(\mathfrak{u}_{\alpha} \cap \mathfrak{A}\right)$. Posons $M_{\alpha}=\mathfrak{m}_{\alpha} \cap G$ et $M_{H, \alpha}=M_{\alpha} \cap H$. Notons $A_{\alpha}$ le centre de $M_{\alpha}$, et posons $A_{H, \alpha}=A_{\alpha} \cap H$.

Soit $\alpha \in \Pi_{n}$. Soient $d m, d u$ et $d \bar{u}$ les mesures de Haar respectivement sur $\mathfrak{m}_{\alpha}$, $\mathfrak{u}_{\alpha}$ et $\overline{\mathfrak{u}}_{\alpha}$ normalisées par $\mathfrak{A}$; et $d m$ et $d u$ les mesures de Haar respectivement sur $M_{\alpha}$ et $U_{\alpha}$ normalisées par $K_{H}$. On note encore $f \mapsto f^{\vee}$ la transformée de Fourier sur $C_{\mathrm{c}}^{\infty}\left(\mathfrak{m}_{\alpha}\right)$ définie par $f^{\vee}(y)=\int_{\mathfrak{m}_{\alpha}} f(m) \overline{\Psi_{A}(y m)} d m$, et $T \mapsto T^{\vee}$ la transformée de Fourier sur $\mathscr{D}\left(\mathfrak{m}_{\alpha}\right)$ définie par $\left\langle f, T^{\vee}\right\rangle=\left\langle f^{\vee}, T\right\rangle\left(f \in C_{\mathrm{c}}^{\infty}\left(\mathfrak{m}_{\alpha}\right)\right)$.

Pour $f \in C_{\mathrm{c}}^{\infty}(A)$, notons $f_{H, \mathfrak{p}_{\alpha}} \in C_{\mathrm{c}}^{\infty}\left(\mathfrak{m}_{\alpha}\right)$ la fonction définie par

$$
f_{H, \mathfrak{p}_{\alpha}}(m)=\iint_{\mathfrak{u}_{\alpha} \times K_{H}} f\left(k^{-1}(m+u) k\right) d u d k
$$

Et pour $T \in \mathscr{D}\left(\mathfrak{m}_{\alpha}\right)$, notons $T_{H, \mathfrak{p}_{\alpha}} \in \mathscr{D}(A)$ la distribution définie par

$$
\left\langle f, T_{H, \mathfrak{p}_{\alpha}}\right\rangle=\left\langle f_{H, \mathfrak{p}_{\alpha}}, T\right\rangle .
$$

Posons $\mu_{\alpha}=\operatorname{vol}\left(\mathfrak{u}_{\alpha} \cap \mathfrak{A}^{1 / d}, d u\right)\left(=\left[\mathfrak{u}_{\alpha} \cap \mathfrak{A}: \mathfrak{u}_{\alpha} \cap \mathfrak{A}^{1 / d}\right]\right)$.

Lemme 2.8. Pour $f \in C_{\mathrm{c}}^{\infty}(A)$, on $a\left(f^{\vee}\right)_{H, \mathfrak{p}_{\alpha}}=\mu_{\alpha}\left(f_{H, \mathfrak{p}_{\alpha}}\right)^{\vee}$. 
Démonstration. Soit $f \in C_{\mathrm{c}}^{\infty}(A)$. Pour $\left(k, m_{1}, u_{1}\right) \in K_{H} \times \mathfrak{m}_{\alpha} \times \mathfrak{u}_{\alpha}$, on a

$$
\begin{aligned}
f^{\vee}\left(k^{-1}\left(m_{1}+u_{1}\right) k\right) & =\int_{A} f\left(k^{-1} g k\right) \overline{\Psi_{A}\left(\left(m_{1}+u_{1}\right) g\right)} d_{A} g \\
& =\int_{\bar{u}_{\alpha}} \overline{\Psi_{A}\left(u_{1} \bar{u}\right)} F_{f, k, m_{1}}(\bar{u}) d \bar{u}
\end{aligned}
$$

avec $F_{f, k, m_{1}}(\bar{u})=\iint_{\mathfrak{m}_{\alpha} \times \mathfrak{u}_{\alpha}} f\left(k^{-1}(\bar{u}+m+u) k\right) \overline{\Psi_{A}\left(m_{1}(m+u)\right)} d m d u$. D'où l'on déduit que

$$
\left(f^{\vee}\right)_{H, \mathfrak{p}_{\alpha}}\left(m_{1}\right)=c \int_{\mathfrak{u}_{\alpha}}\left\{\int_{\bar{u}_{\alpha}} \overline{\Psi_{A}(u \bar{u})} F_{f, m_{1}}(\bar{u}) d \bar{u}\right\} d u
$$

avec $F_{f, m_{1}}(\bar{u})=\int_{K_{H}} F_{f, k, m_{1}}(\bar{u}) d k$. Comme $F_{f, m_{1}} \in C_{\mathrm{c}}^{\infty}\left(\overline{\mathfrak{u}}_{\alpha}\right)$, il existe un entier $a$ tel que $F_{f, m_{1}} \in C_{\mathrm{c}}\left(\overline{\overline{\mathfrak{u}}}_{\alpha} /\left(\overline{\mathfrak{u}}_{\alpha} \cap \mathfrak{U}^{a}\right)\right)$. Un calcul facile montre alors que

$$
\begin{aligned}
\left(f^{\vee}\right)_{H, \mathfrak{p}_{\alpha}}\left(m_{1}\right) & =F_{f, m_{1}}(0) \iint_{\left(\mathfrak{u}_{\alpha} \cap \mathfrak{A}^{(1 / d)-a}\right) \times\left(\overline{\mathfrak{u}}_{\alpha} \cap \mathfrak{A}^{a}\right)} \overline{\Psi_{A}(u \bar{u})} d u d \bar{u} \\
& =F_{f, m_{1}}(0) \operatorname{vol}\left(\mathfrak{u}_{\alpha} \cap \mathfrak{A}^{(1 / d)-a}, d u\right) \operatorname{vol}\left(\overline{\mathfrak{u}}_{\alpha} \cap \mathfrak{A}^{a}, d \bar{u}\right) .
\end{aligned}
$$

Or $F_{f, m_{1}}(0)=\left(f_{H, \mathfrak{p}_{\alpha}}\right)^{\vee}\left(m_{1}\right), \operatorname{vol}\left(\mathfrak{u}_{\alpha} \cap \mathfrak{A}^{(1 / d)-a}, d u\right)=\mu_{\alpha} \operatorname{vol}\left(\mathfrak{u}_{\alpha} \cap \mathfrak{A}^{a}, d u\right)^{-1}$ et $\operatorname{vol}\left(\overline{\mathfrak{u}}_{\alpha} \cap \mathfrak{A}^{a}, d \bar{u}\right)=\operatorname{vol}\left(\mathfrak{u}_{\alpha} \cap \mathfrak{A}^{a}, d u\right)$. D'où le lemme.

Pour tout sous-groupe de Cartan $\Gamma \subset G$, on note $A_{\Gamma}$ le sous-tore $F$-déployé maximal de $\Gamma, d_{A_{\Gamma}} a$ la mesure de Haar sur $A_{\Gamma}$ normalisée par le sous-groupe compact maximal de $A_{\Gamma}$, et $d_{\Gamma} \gamma$ la mesure de Haar sur $\Gamma$ telle que $\operatorname{vol}\left(A_{\Gamma_{H}} \backslash \Gamma_{H}, \frac{d_{\Gamma} \gamma}{d_{A_{\Gamma}} a}\right)=$ 1 ; où l'on a posé $\Gamma_{H}=\Gamma \cap H$ et $A_{\Gamma_{H}}=A_{\Gamma} \cap H$. Si $\gamma \in A^{\prime}$, alors le centralisateur $\Gamma=G_{\gamma}$ est un sous-groupe de Cartan de $G$, et l'on note $\Phi_{H}(\cdot, \gamma)$ la distribution $H$-invariante sur $A$ définie par

$$
\Phi_{H}(f, \gamma)=\int_{\Gamma_{H} \backslash H} f\left(g^{-1} \gamma g\right) \frac{d h}{d_{\Gamma} \gamma} .
$$

Puisque l'orbite $\widehat{O}_{H}(\gamma)$ est fermée dans $A$, l'intégrale ci-dessus est absolument convergente. Notons que pour $g \in G$ et $\gamma \in A^{\prime}$, on a

$$
\Phi_{H}\left(\cdot, g^{-1} \gamma g\right)=\operatorname{Ad} g^{-1}\left(\Phi_{H}(\cdot, \gamma)\right) .
$$

De la même manière, si $\gamma \in \mathfrak{m}_{\alpha} \cap A^{\prime}$ pour un $\alpha \in \Pi_{n}$, on note $\Phi_{M_{H, \alpha}}(\cdot, \gamma)$ la distribution $M_{H, \alpha}$-invariante sur $\mathfrak{m}_{\alpha}$ définie par

$$
\Phi_{M_{H, \alpha}}(f, \gamma)=\int_{\Gamma_{H} \backslash M_{H, \alpha}} f\left(m^{-1} \gamma m\right) \frac{d m}{d_{\Gamma} \gamma} .
$$

Lemme 2.9. Soit $T \in \mathscr{D}\left(\mathfrak{m}_{\alpha}\right)$ une distribution $M_{H, \alpha}$-invariante et localement intégrable. Alors la distribution $T_{H, \mathfrak{p}_{\alpha}}$ est $H$-invariante et localement intégrable. Si de 
plus $T$ est une distribution localement constante sur $\mathfrak{m}_{\alpha} \cap A^{\prime}$, alors la distribution $T_{H, \mathfrak{p}_{\alpha}}$ est localement constante sur $A^{\prime}$.

Démonstration. Mutatis mutandis, la démonstration est celle du lemme 1.8 de [Lemaire 2004]. Pour $f \in C_{\mathrm{c}}^{\infty}(H)$, on a la formule d'intégration

$$
\int_{H} f(h) d h=\iiint_{M_{H, \alpha} \times U_{\alpha} \times K_{H}} f(m u k) d m d u d k .
$$

Grâce à la cette formule, pour toute fonction $f \in C_{\mathrm{c}}^{\infty}(A)$ et tout élément $\gamma \in$ $\mathfrak{m}_{\alpha} \cap A^{\prime}$, on montre la formule de descente

$$
\Phi_{M_{H, \alpha}}\left(f_{H, \mathfrak{p}_{\alpha}}, \gamma\right)=\left|\eta_{\mathfrak{m}_{\alpha} \backslash A}(\gamma)\right|^{1 / 2} \Phi_{H}(f, \gamma)
$$

où $\eta_{\mathfrak{m}_{\alpha} \backslash A}(\gamma)=\operatorname{det}_{F}\left(\operatorname{ad} \gamma ; \mathfrak{m}_{\alpha} \backslash A\right)$. Comme dans la démonstration de [Lemaire 2004, 1.8], l'égalité (2.10), injectée dans les formules d'intégration de Weyl pour $M_{H, \alpha}$ et $H$, implique toutes les assertions du lemme.

Pour $\alpha \in \Pi_{n}$, on note $\mathfrak{m}_{\alpha, \mathrm{e}}^{\prime}$ l'ensemble des $\gamma \in \mathfrak{m}_{\alpha} \cap A^{\prime}$ tels que le centralisateur $G_{\gamma}$ est compact modulo $A_{\alpha}$. On a la décomposition

$$
A^{\prime}=\coprod_{\alpha \in \Pi_{n}}{ }^{G}\left\{\mathfrak{m}_{\alpha, \mathrm{e}}^{\prime}\right\}=\coprod_{\alpha \in \Pi_{n}}{ }^{H}\left\{\mathfrak{m}_{\alpha, \mathrm{e}}^{\prime}\right\} .
$$

Soient $\alpha \in \Pi_{n}$ et $\gamma \in \mathfrak{m}_{\alpha, \mathrm{e}}^{\prime}$. D'après 2.8 et (2.10), pour $f \in C_{\mathrm{c}}^{\infty}(A)$, on a

$$
\Phi_{M_{H, \alpha}}\left(\left(f_{H, \mathfrak{p}_{\alpha}}\right)^{\vee}, \gamma\right)=\mu_{\alpha}^{-1}\left|\eta_{\mathfrak{m}_{\alpha} \backslash A}(\gamma)\right|^{1 / 2} \Phi_{H}\left(f^{\vee}, \gamma\right) .
$$

Par conséquent 2.9, si la distribution $\Phi_{M_{H, \alpha}}(\cdot, \gamma)^{\vee} \in J_{M_{H, \alpha}}\left(\mathfrak{m}_{\alpha}\right)$ est localement intégrable sur $\mathfrak{m}_{\alpha}$, alors la distribution $\Phi_{H}(\cdot, \gamma)^{\vee} \in J_{H}(A)$ est localement intégrable sur $A$. Rappelons que d'après la proposition 2.7, on sait déjà que pour $y \in A^{\prime}$, la distribution $\Phi_{H}(\cdot, y)^{\vee}$ est localement constante sur $A^{\prime}$. D'où le

Lemme 2.13. On suppose que pour tout $\gamma \in A_{\mathrm{e}}^{\prime}$, la distribution $\Phi_{H}(\cdot, \gamma)^{\vee}$ est localement intégrable sur $A$ (resp. intégrable au voisinage de 0 dans $A$ ). Alors pour tout $y \in A^{\prime}$, la distribution $\Phi_{H}(\cdot, y)^{\vee}$ est localement intégrable sur A (resp. intégrable au voisinage de 0 dans $A)$.

Démonstration. Par récurrence sur la dimension de $A$, grâce à (2.11), (2.12) et 2.9. Notons que si $\alpha \in \Pi_{n}$ et $f \in C_{\mathrm{c}}^{\infty}\left(\mathfrak{A}^{b}\right)$ pour un $b \in \mathbb{Z}$, alors $f_{H, \mathfrak{p}_{\alpha}} \in C_{\mathrm{c}}^{\infty}\left(\mathfrak{m}_{\alpha} \cap \mathfrak{A}^{b}\right)$.

Intégrabilité des distributions $\Phi_{H}(\cdot, \gamma)^{\vee}\left(\gamma \in A_{\mathrm{e}}^{\prime}\right)$ au voisinage de 0 dans $A$. Soit $d z$ la mesure de Haar sur $Z\left(=F^{\times}\right)$normalisée par $\mathfrak{o}_{F}^{\times}$. Pour $\gamma \in A_{\mathrm{e}}^{\prime}$, on a donc $\Phi_{H}(f, \gamma)=\int_{Z_{H} \backslash H} f\left(h^{-1} \gamma h\right)(d h / d z)$, où $f \in C_{\mathrm{c}}^{\infty}(A)$.

Fixons un système de représentants $\mathscr{C}_{H, \mathrm{e}}(G)$ dans $G$ des classes de $H$-conjugaison de sous-groupes de Cartan elliptiques de $G$. 
Lemme 2.14 [Harish-Chandra 1978, part I, §2, lemma 1]. Soit $\theta \in C_{\mathrm{c}}^{\infty}\left(A_{\mathrm{e}}^{\prime}\right)$. Alors pour $f \in C_{\mathrm{c}}^{\infty}(A)$, on a

$$
\int_{Z_{H} \backslash H}\left|\int_{A} f(g) \theta^{\vee}\left(h^{-1} g h\right) d_{A} g\right| \frac{d h}{d z}<+\infty .
$$

Démonstration. D'après le début de la démonstration du corollaire 2.6, il existe un sous-ensemble fini $\left\{\Gamma_{1}, \ldots, \Gamma_{s}\right\} \subset \mathscr{C}_{H, \mathrm{e}}(G)$ tel que $\operatorname{Supp}(\theta) \subset \bigcup_{i=1}^{s}{ }^{H}\left\{L\left(\Gamma_{i}\right)\right\}$. Pour $i=1, \ldots, s$, l'intersection $X_{i}={ }^{H}\left\{L\left(\Gamma_{i}\right)\right\} \cap \operatorname{Supp}(\theta) \subset{ }^{H}\left\{L\left(\Gamma_{i}\right)^{\prime}\right\}$ est ouverte et compacte (rappelons que ${ }^{H}\left\{L\left(\Gamma_{i}\right)^{\prime}\right\}$ est ouvert et fermé dans $A_{\mathrm{e}}^{\prime}$ ). On a $\theta=$ $\theta_{1}+\cdots+\theta_{s}$ avec $\theta_{i}=\left.\theta\right|_{X_{i}} \in C_{\mathrm{c}}^{\infty}\left({ }^{H}\left\{L\left(\Gamma_{i}\right)^{\prime}\right\}\right)$. On peut donc supposer que $\theta \in$ $C_{\mathrm{c}}^{\infty}\left({ }^{H}\left\{L(\Gamma)^{\prime}\right\}\right)$ pour un $\Gamma \in \mathscr{C}_{H, \mathrm{e}}(G)$. Soit $d_{L(\Gamma)} \gamma$ la mesure de Haar sur $L(\Gamma)$ définie par $\left|\operatorname{det}_{F}(y \mapsto \gamma y ; L(\Gamma))\right| d_{\Gamma} \gamma=d_{L(\Gamma)} \gamma$.

Pour $h \in H$, on a $\int_{A} f(g) \theta^{\vee}\left(h^{-1} g h\right) d_{A} g=\int_{A} f^{\vee}(g) \theta^{h}(g) d_{A} g$ avec $\theta^{h}(g)=$ $\theta\left(h^{-1} g h\right)$; et puisque $\operatorname{Supp}(\theta) \subset{ }^{H}\{L(\Gamma)\}$, la formule d'intégration de Weyl pour $G$ implique que

$$
\int_{A} f^{\vee}(g) \theta\left(h^{-1} g h\right) d_{A} g=c \int_{L(\Gamma)^{\prime}}|\eta(\gamma)| \Theta_{H, \gamma}\left(f^{\vee} \cdot \theta^{h}\right) d_{L(\Gamma) \gamma}
$$

avec

$$
c=\left|\mathrm{N}_{H}\left(\Gamma_{H}\right) / \Gamma_{H}\right|^{-1} \quad\left(\text { où } \mathrm{N}_{H}\left(\Gamma_{H}\right)=\left\{h \in H: h^{-1} \Gamma_{H} \gamma=\Gamma_{H}\right\}\right)
$$

et

$$
\eta(\gamma)=\operatorname{det}_{F}(\operatorname{ad} \gamma ; L(\Gamma) \backslash A)
$$

On a donc

$$
\begin{aligned}
\int_{Z_{H} \backslash H} \mid \int_{A} f(g) \theta^{\vee}\left(h^{-1} g h\right) & d_{A} g \mid \frac{d h}{d z} \\
& \leq c \int_{L(\Gamma)^{\prime}}|\eta(\gamma)|\left(\int_{Z_{H} \backslash H}\left|\Theta_{H, \gamma}\left(f^{\vee} \cdot \theta^{h}\right)\right| \frac{d h}{d z}\right) d_{L(\Gamma) \gamma} \\
& \leq c \int_{L(\Gamma)^{\prime}}|\eta(\gamma)| \Theta_{H, \gamma}\left(\left|f^{\vee}\right|\right) \Theta_{H, \gamma}(|\theta|) d_{L(\Gamma)} \gamma .
\end{aligned}
$$

La fonction $\gamma \mapsto|\eta(\gamma)|$ est localement constante sur $L(\Gamma)^{\prime}$; et d'après [HarishChandra 1980], pour toute fonction $h \in C_{\mathrm{c}}^{\infty}(A)$, la fonction $\gamma \mapsto \Theta_{H, \gamma}(h)$ est localement constante sur $L(\Gamma)^{\prime}$. Comme $\Theta_{H}, .(|\theta|) \in C_{\mathrm{c}}^{\infty}\left(L(\Gamma)^{\prime}\right)$, la dernière intégrale ci-dessus est convergente. D'où le lemme.

Pour $\theta \in C_{\mathrm{c}}^{\infty}\left(A_{\mathrm{e}}^{\prime}\right)$, notons $T_{H, \theta}$ la distribution sur $A$ définie par la lemme 2.14

$$
\left\langle f, T_{H, \theta}\right\rangle=\int_{Z_{H} \backslash H}\left(\int_{A} f(g) \theta^{\vee}\left(h^{-1} g h\right) d_{A} g\right) \frac{d h}{d z} .
$$


On a clairement (cf. la démonstration ci-dessus) : $T_{H, \theta} \in J_{H}\left(X_{\theta}\right)^{\vee}$ avec $X_{\theta}=$ $\overline{{ }^{H}\{\operatorname{Supp}(\theta)\}}$. Et puisque $X_{\theta}$ est contenu dans $\varpi^{k} \Xi$ pour un $k \in \mathbb{Z}$, on a $T_{H, \theta} \in$ $J_{H}^{*}(A)^{\vee}$. En particulier (voir la proposition 2.7), la distribution $T_{H, \theta}$ est localement constante sur $A^{\prime}$.

Proposition 2.15. Soit $\theta \in C_{\mathrm{c}}^{\infty}\left(A_{\mathrm{e}}^{\prime}\right)$. Alors la distribution $T_{H, \theta}$ est intégrable au voisinage de 0 dans $A$.

Démonstration. Soit $T_{G, \theta}^{+}$la distribution sur $A$ définie par le lemme 2.14

$$
\begin{aligned}
\left\langle f, T_{G, \theta}^{+}\right\rangle & =\int_{Z \backslash G}\left|\int_{A} f(x) \theta^{\vee}\left(g^{-1} x g\right) d_{A} x\right| \frac{d g}{d z} \\
& =\int_{Z \backslash G}\left|\int_{A} f^{\vee}(x) \theta\left(g^{-1} x g\right) d_{A} x\right| \frac{d g}{d z} .
\end{aligned}
$$

Puisque $T_{G, \theta}^{+} \in J_{G}^{*}(A)^{\vee}$, d'après la proposition 1.6, il existe un entier $b$ et des constantes $c_{\alpha}\left(\alpha \in \Pi_{n}\right)$ tels que

$$
\mathbf{1}_{\mathfrak{A}^{b}} \cdot\left(T_{G, \theta}^{+}-\sum_{\alpha \in \Pi_{n}} c_{\alpha} \Theta_{\alpha}^{\vee}\right)=0 .
$$

Par conséquent [Lemaire 2004, 1.10/3], la distribution $\mathbf{1}_{\mathfrak{U}^{b}} \cdot T_{G, \theta}^{+}$est intégrable sur $A$. Soit $\mathscr{K}$ un sous-groupe ouvert compact de $H$. D'après la proposition 2.7, pour $y \in A^{\prime}$, on a

$$
T_{G, \theta}^{+}(y)=\int_{Z \backslash G}\left|\int_{A} \bar{\Psi}_{A, y}^{\mathscr{K}}(-x) \theta\left(g^{-1} x g\right) d_{A} x\right| \frac{d g}{d z} .
$$

On en déduit que la fonction

$$
A^{\prime} \times\left(Z_{H} \backslash H\right) \rightarrow \mathbb{C},(y, h) \mapsto \int_{A} \bar{\Psi}_{A, y}^{\mathscr{K}}(-x) \theta\left(h^{-1} x h\right) d_{A} x
$$

est intégrable sur $\mathfrak{A}^{b} \times\left(Z_{H} \backslash H\right)$ par rapport à la mesure produit. On peut donc appliquer le théorème de Fubini : pour presque tout $y \in \mathfrak{A}^{b}$, la fonction $Z_{H} \backslash H \rightarrow$ $\mathbb{C}, h \mapsto F_{y}(h)=\int_{A} \bar{\Psi}_{A, y}^{\mathscr{Y}}(-x) \theta\left(h^{-1} x h\right) d_{A} x$ est intégrable sur $Z_{H} \backslash H$; pour toute fonction $f \in C_{\mathrm{c}}^{\infty}\left(\mathfrak{A}^{b}\right)$, la fonction $F_{f}: A \rightarrow \mathbb{C}$ définie presque partout par $F_{f}(y)=$ $f(y) \int_{Z_{H} \backslash H} F_{y}(h)(d h / d z)$ est intégrable sur $A$; et l'on a $\left\langle f, T_{H, \theta}\right\rangle=\int_{A} F_{f}(y) d_{A} y$. Puisque pour tout $y \in A^{\prime}$ on a $T_{H, \theta}(y)=\int_{Z_{H} \backslash H} F_{y}(h)(d h / d z)$ (toujours par la proposition 2.7), la proposition est démontrée.

Proposition 2.16. Soit $\gamma \in A_{\mathrm{e}}^{\prime}$. Alors il existe une fonction $\theta \in C_{\mathrm{c}}^{\infty}\left(A_{\mathrm{e}}^{\prime}\right)$ telle que $\Phi_{H}(\cdot, \gamma)^{\vee}=T_{H, \theta}$. 
Démonstration. Une simple adaptation de la démonstration de [Harish-Chandra 1980, théorème 4] montre que pour tout $v \in \frac{1}{d} \mathbb{Z}$, l'application $A_{\mathrm{e}}^{\prime} \rightarrow j^{v}\left(J_{H}(A)\right)$, $y \mapsto j^{v}\left(\Phi_{H}(\cdot, y)\right)$ est localement constante.

Posons $\Gamma=G_{\gamma}$. Soit $f \in C_{\mathrm{c}}^{\infty}(A)$, et soit $\omega$ un voisinage ouvert compact de $\gamma$ dans $L(\Gamma)^{\prime}$ tel que $\Phi_{H}\left(f^{\vee}, y\right)=\Phi_{H}\left(f^{\vee}, \gamma\right)$ pour tout $y \in \omega$. Puisque l'application $\zeta: H \times \omega \rightarrow{ }^{H}\left\{L(\Gamma)^{\prime}\right\},(h, y) \mapsto h^{-1} y h$ est partout submersive, il existe une unique application linéaire surjective [Harish-Chandra 1970, theorem 11]

$$
C_{\mathrm{c}}^{\infty}(H \times \omega) \rightarrow C_{\mathrm{c}}^{\infty}(\zeta(H \times \omega)), \varphi \mapsto \varphi^{\zeta}
$$

telle que

$$
\iint_{H \times \omega} \varphi(h, y) \Phi \circ \zeta(h, y) d h d_{L(\Gamma)} y=\int_{A} \varphi^{\zeta}(g) \Phi(g) d_{A} g
$$

pour toutes fonctions $\varphi \in C_{\mathrm{c}}^{\infty}(H \times \omega)$ et $\Phi \in C_{\mathrm{c}}^{\infty}(\zeta(H \times \omega))$; où $d_{L(\Gamma)} y$ est la mesure de Haar sur $L(\Gamma)$ définie comme dans la démonstration du lemme 2.14. Choisissons une fonction $\varphi \in C_{\mathrm{c}}^{\infty}(H \times \omega)$ telle que $\iint_{H \times \omega} \varphi(h, y) d h d_{L(\Gamma)} y=1$, posons $\theta=\varphi^{\zeta}$, et notons $\varphi_{\zeta} \in C_{\mathrm{c}}^{\infty}(\omega)$ la fonction $y \mapsto \int_{H} \varphi(h, y) d h$. Alors $\theta \in$ $C_{\mathrm{c}}^{\infty}\left(A^{\prime}\right)$, et l'on a

$$
\begin{aligned}
\left\langle f, T_{H, \theta}\right\rangle & =\int_{Z_{H} \backslash H}\left(\int_{A} f^{\vee}\left(h g h^{-1}\right) \varphi^{\zeta}(g) d_{A} g\right) \frac{d h}{d z} \\
& =\int_{Z_{H} \backslash H}\left(\iint_{H \times \omega} f^{\vee}\left(h h^{\prime-1} y h^{\prime} h^{-1}\right) \varphi\left(h^{\prime}, y\right) d h^{\prime} d_{L(\Gamma)} y\right) \frac{d h}{d z} .
\end{aligned}
$$

Mais puisque

$$
\int_{Z_{H} \backslash H}\left(\iint_{H \times \omega}\left|f^{\vee}\left(h h^{\prime-1} y h^{\prime} h^{-1}\right)\right|\left|\varphi\left(h^{\prime}, y\right)\right| d h^{\prime} d_{L(\Gamma)} y\right) \frac{d h}{d z}<+\infty
$$

(cf. la démonstration du lemme 2.14), on peut appliquer le théorème de Fubini. On obtient :

$$
\begin{aligned}
\left\langle f, T_{H, \theta}\right\rangle & =\int_{H \times \omega} \Phi_{H}\left(f^{\vee}, y\right) \varphi\left(h^{\prime}, y\right) d h^{\prime} d_{L(\Gamma)} y \\
& =\int_{\omega} \Phi_{H}\left(f^{\vee}, y\right) \varphi_{\zeta}(y) d_{L(\Gamma)} y \\
& =\Phi_{H}\left(f^{\vee}, \gamma\right)
\end{aligned}
$$

$\operatorname{car} \int_{\omega} \varphi_{\zeta}(y) d_{L(\Gamma)} y=1$.

Corollaire 2.17. La distribution

$$
\Phi_{H}(\cdot, \gamma)^{\vee}
$$

est intégrable au voisinage de 0 dans $A$. 
Germes de Shalika au voisinage de 0 dans $A$. Puisque $H$ est ouvert dans $G$, pour $y \in A$, le centralisateur $H_{y}$ est ouvert dans $G_{y}$. D'après [Laumon 1996, 4.8.6] et la démonstration de [Lemaire 2004, 1.10/1], pour $y \in A$, le centralisateur $G_{y}$ est unimodulaire; donc $H_{y}$ est unimodulaire.

Lemme 2.18. Soient $y \in A$ et $d h_{y}$ une mesure de Haar sur $H_{y}$. Pour toute fonction $f \in C_{\mathrm{c}}^{\infty}(A)$, l'intégrale orbitale

$$
\Phi_{H}\left(f, y, d h_{y}\right)=\int_{H_{y} \backslash H} f\left(h^{-1} y h\right) \frac{d h}{d h_{y}}
$$

est absolument convergente; i.e., $\Phi_{H}\left(\cdot, y, d h_{y}\right) \in J_{H}(A)$.

Démonstration. Puisque $Z H$ est un sous-groupe d'indice fini de $G, H_{y} \backslash H$ est un sous-groupe d'indice fini de $G_{y} \backslash G$. On peut donc supposer que $H=G$. Les composantes $F$-irréductibles du polynômes caractéristique réduit de $y$ définissent, comme en [Lemaire 1997, 2.8], un sous-groupe de Lévi $M^{\prime}$ de $G$ et un sous-groupe parabolique $P^{\prime \prime}$ de $M^{\prime}$ [Lemaire 2004, section 5]). Soit $U^{\prime \prime}$ le radical unipotent de $P^{\prime \prime}$. Choisissons une décomposition de Lévi $P^{\prime \prime}=M U^{\prime \prime}$, et un sous-groupe parabolique $P^{\prime}$ de $G$ de composante de Lévi $M^{\prime}$. Soit $U^{\prime}$ le radical unipotent de $P^{\prime}$. Alors $P=P^{\prime \prime} U^{\prime}$ est un sous-groupe parabolique de $G$ de radical unipotent $U=U^{\prime \prime} U^{\prime}$, et $P=M U$ est une décomposition de Lévi de $P$. Notons $\mathfrak{m}^{\prime}, \mathfrak{p}^{\prime \prime}$ (etc.) les sous- $F$-algèbres de $A$ correspondant à $M^{\prime}, P^{\prime \prime}$ (etc.). Par construction, on a $y \in \mathfrak{p}^{\prime \prime}$ et $G_{y} \subset P^{\prime \prime}$. Écrivons $y=x+u$ avec $x \in \mathfrak{m}$ et $u \in \mathfrak{u}^{\prime \prime}$. Comme dans [Laumon 1996, 4.8.4], on montre que l'orbite $\mathfrak{O}_{M}(x)$ est fermée dans $\mathfrak{m}$, et que $\overline{O_{P^{\prime \prime}}(y)}=\widehat{O}_{M}(x) \oplus \mathfrak{u}^{\prime \prime}$. On en déduit (voir [Lemaire 1997, 2.8.2]) que $\overline{O_{P}(y)}=$ $\mathfrak{O}_{M}(x) \oplus \mathfrak{u}$. Le reste de la démonstration est une simple adaptation de celle de la proposition 3.2.2 de [Lemaire 1997]. Plus précisément : quitte à remplacer $y$ par $h^{-1} y h$ (resp. $P$ par $h^{-1} P h, M$ par $h^{-1} M h$ ) pour un $h \in H$, on peut supposer que $P=P_{\alpha}$ et $M=M_{\alpha}$ pour un $\alpha \in \Pi_{n}$. Alors on montre qu'il existe une (unique) mesure de Haar $d m_{x}$ sur $\left(M_{H, \alpha}\right)_{x}$ telle que pour toute fonction $f \in C_{\mathrm{c}}^{\infty}(A)$, on a la formule de descente

$$
\Phi_{H}\left(f, y, d h_{y}\right)=\Phi_{M_{H, \alpha}}\left(f_{H, \mathfrak{p}_{\alpha}}, x, d m_{x}\right)
$$

avec

$$
\Phi_{M_{H, \alpha}}\left(\phi, x, d m_{x}\right)=\int_{\left(M_{H, \alpha}\right)_{x} \backslash M_{H, \alpha}} \phi\left(m^{-1} x m\right) \frac{d m}{d m_{x}} \quad\left(\phi \in C_{\mathrm{c}}^{\infty}\left(\mathfrak{m}_{\alpha}\right)\right) .
$$

Puisque la $M_{H, \alpha}$-orbite $\mathcal{O}_{M_{H, \alpha}}(x)$ est fermée dans $\mathfrak{m}_{\alpha}$, l'intégrale ci-dessus est absolument convergente.

Pour chaque élément $y \in A$, choisissons une mesure de Haar $d h_{y}$ sur $H_{y}$, et posons $\Phi_{H}(\cdot, y)=\Phi_{H}\left(\cdot, y, d h_{y}\right) \in J_{H}(A)$. On suppose que ces mesures $d h_{y}$ $(y \in A)$ vérifient les propriétés suivantes : 
- Pour tout $\left(y, y^{\prime}, g\right) \in A \times A \times G$ tel que $g^{-1} y g=y^{\prime}, d h_{y^{\prime}}$ est la mesure de Haar sur $H_{y^{\prime}}$ déduite de $d h_{y}$ via l'isomorphisme de groupes $H_{y} \rightarrow H_{y^{\prime}}, x \mapsto g^{-1} x g$. Ainsi, pour tout $(y, g) \in A \times H$, on a $\Phi_{H}\left(\cdot, g^{-1} y g\right)=\operatorname{Ad}^{-1}\left(\Phi_{H}(\cdot, y)\right)$.

- Pour $y \in A^{\prime}$, la distribution $\Phi_{H}(\cdot, y)$ coïncide avec celle définie précédemment; i.e., $d h_{y}=\left.d_{\Gamma} y\right|_{\Gamma_{H}}$ où $\Gamma=G_{y}$.

- Pour tout $(\alpha, \bar{x}) \in \Pi_{H, n}$, on a $\Phi_{H}\left(\cdot, n_{\alpha, x}\right)=\Theta_{\alpha, \bar{x}}$.

Ordonnons les $H$-orbites de $\mathfrak{N}$ de manière à former une décomposition standard; i.e., écrivons $\mathfrak{N}=\coprod_{i=0}^{s} \mathrm{O}_{i}$ avec

- pour $i=0, \ldots s, O_{i}$ est une $H$-orbite nilpotente,

- pour $i=0, \ldots, s-1, \operatorname{dim}\left(O_{i}\right) \leq \operatorname{dim}\left(O_{i+1}\right)$.

Pour $k=0, \ldots, s$, posons $\mathfrak{N}_{k}=\coprod_{i=0}^{k} \mathcal{O}_{i}$. Puisque $Z H$ est un sous-groupe d'indice fini de $G$, le corollaire 5.2 de [Lemaire 2004] reste vrai si l'on remplace $G$ par $H$. On en déduit que pour $k=0, \ldots, s, \mathfrak{N}_{k}$ est fermé dans $A$ et $O_{k}$ est ouvert dans $\mathfrak{N}_{k}$. Pour $(\alpha, \bar{x}) \in \Pi_{H, n}$, on note $i(\alpha, \bar{x})$ l'unique $i \in\{0, \ldots, s\}$ tel que $\mathbb{O}_{i}=\mathscr{O}_{\alpha, \bar{x}}$.

Lemme 2.20. Pour tout $v \in \frac{1}{d} \mathbb{Z}$, il existe un jeu de fonctions $\left\{f_{k}\right\}_{k=0}^{s} \subset C_{\mathrm{c}}^{\infty}\left(\mathfrak{A}^{v}\right)$ tel que

(1) $\operatorname{Supp}\left(f_{k}\right) \cap \mathfrak{N}_{k} \subset \mathfrak{O}_{k}$,

(2) $\left\langle f_{k}, \Theta_{i}\right\rangle=\delta_{k, i}(i=0, \ldots, s)$.

Démonstration. Elle est identique à celle du lemme 3.5.1 de [Lemaire 1997].

Toute fonction $\Phi$ définie au voisinage de 0 dans $A$, induit un germe de fonctions au point 0 dans $A$, que l'on note $[\Phi]_{0}$ (cf. [Lemaire 1997, 3.5]).

Proposition 2.21. Il existe une unique famille $\left\{\Gamma_{\alpha, \bar{x}}:(\alpha, \bar{x}) \in \Pi_{H, n}\right\}$ de germes de fonctions au point 0 dans $A$, telle que

$$
\left[\Phi_{H}(f, \cdot)\right]_{0}=\sum_{(\alpha, \bar{x})}\left\langle f, \Theta_{\alpha, \bar{x}}\right\rangle \Gamma_{\alpha, \bar{x}} \quad\left(f \in C_{\mathrm{c}}^{\infty}(A)\right)
$$

où $(\alpha, \bar{x})$ parcourt les éléments de $\Pi_{H, n}$. De plus, ces germes sont donnés par $\Gamma_{\alpha, \bar{x}}=\left[\Phi_{H}\left(f_{i(\alpha, \bar{x})}, \cdot\right)\right]_{0}$ pour tout jeu de fonctions $\left\{f_{i}\right\}_{i=0}^{s} \subset C_{\mathrm{c}}^{\infty}(A)$ vérifiant les conditions (1) et (2) du lemme 2.20 .

Démonstration. Elle est identique à celle de la proposition 3.5.2 de [Lemaire 1997].

Remarque 2.22. D'après (2.10) et le début de la démonstration de 2.16, pour tout $v \in \frac{1}{d} \mathbb{Z}$, l'application $A^{\prime} \rightarrow j^{v}\left(J_{H}(A)\right), y \mapsto j^{v}\left(\Phi_{H}(\cdot, y)\right)$ est localement constante. On en déduit que les germes $\Gamma_{\alpha, \bar{x}}\left((\alpha, \bar{x}) \in \Pi_{H, n}\right)$ sont localement constants sur $A^{\prime}$.

Le groupe $G$ opère naturellement (à gauche) sur $\Pi_{H, n}$ : pour $g \in G$ et $(\alpha, \bar{x})$, on pose $g \cdot(\alpha, \bar{x})=(\alpha, g \cdot \bar{x})$, avec $g \cdot \bar{x}=\delta_{\alpha}\left(g x g^{-1}\right)$ (cf. la section 1 pour la définition de $\left.\delta_{\alpha}\right)$. Puisque pour tout $(y, g) \in G \times A$, on a $\Phi_{H}\left(\cdot, g^{-1} y g\right)=\operatorname{Ad} g^{-1}\left(\Phi_{H}(\cdot, y)\right)$, 
la propriété d'unicité dans 2.21 implique que pour tous $(\alpha, \bar{x}) \in \Pi_{H, n}$ et $g \in G$, on a $\operatorname{Ad}^{*} g\left(\Gamma_{\alpha, \bar{x}}\right)\left(=\Gamma_{\alpha, \bar{x}} \circ \operatorname{Ad}^{-1}\right)=\Gamma_{\alpha, g \cdot \bar{x}}$.

Pour $y \in A$, notons $d \tilde{g}_{y}$ l'unique mesure de Haar sur $G_{y}$ prolongeant $d h_{y}$, et posons $d g_{y}=\left(\Delta_{H} \Delta_{y}: \Delta_{H}\left(F^{\times}\right)^{n}\right)^{-1} d \tilde{g}_{y}$ avec $\Delta_{y}=\operatorname{det}^{\prime}\left(G_{y}\right)$. Soit $\Phi_{G}(\cdot, y)(y \in$ $A$ ) la distribution $G$-invariante sur $A$ définie par $\Phi_{G}(f, y)=\int_{G_{y} \backslash G} f\left(g^{-1} y g\right) \frac{d g}{d g_{y}}$. Alors 2.21, il existe une unique famille $\left\{\Gamma_{\alpha}: \alpha \in \Pi_{n}\right\}$ de germes de fonctions au point 0 dans $A$, telle que

$$
\left[\Phi_{G}(f, \cdot)\right]_{0}=\sum_{\alpha \in \Pi_{n}} \Phi_{G}\left(f, n_{\alpha}\right) \Gamma_{\alpha} \quad\left(f \in C_{\mathrm{c}}^{\infty}(A)\right) .
$$

Puisque

$$
\Phi_{G}(\cdot, y)=\sum_{g \in H Z \backslash G} \Phi_{H}\left(\cdot, g^{-1} y g\right) \quad(y \in A)
$$

et

$$
\Phi_{G}\left(\cdot, n_{\alpha}\right)=\sum_{g \in H Z \backslash G} \Phi_{H}\left(\cdot, g^{-1} n_{\alpha} g\right) \quad\left(\alpha \in \Pi_{n}\right),
$$

on a (calcul facile)

$$
\Gamma_{\alpha}=\sum_{\bar{x}} \Gamma_{\alpha, \bar{x}} \quad\left(\alpha \in \Pi_{n}\right)
$$

où $\bar{x}$ parcourt les éléments de $\Delta_{H} \Delta_{\alpha} \backslash F^{\times}$.

Choisissons un jeu de fonctions $\left\{f_{i}\right\}_{i=0}^{s} \subset C_{\mathrm{c}}^{\infty}(A)$ vérifiant les conditions (1) et (2) du lemme 2.20, et pour $(\alpha, \bar{x}) \in \Pi_{H, n}$, notons $\Gamma_{\alpha, \bar{x}}$ la fonction sur $A$ définie par $\Gamma_{\alpha, \bar{x}}(g)=\Phi_{G}\left(f_{i(\alpha, \bar{x})}, g\right)$. D'après 2.21 , on a $\left[\Gamma_{\alpha, \bar{x}}\right]_{0}=\Gamma_{\alpha, \bar{x}}$; et fixée une fonction $f \in C_{\mathrm{c}}^{\infty}(A)$, il existe un voisinage ouvert $H$-invariant $\mathscr{V}=\mathscr{V}\left(f,\left\{f_{i}\right\}_{i=0}^{s}\right)$ de 0 dans $A$ tel que $\left.\left(\Phi_{H}(f,)-.\sum_{(\alpha, \bar{x})}\left\langle f, \Theta_{\alpha, \bar{x}}\right\rangle \Gamma_{\alpha, \bar{x}}\right)\right|_{\gamma}=0$. La proposition suivante précise ce résultat.

Proposition 2.23. Soit $v \in \frac{1}{d} \mathbb{Z}$. Il existe un $a=a\left(v,\left\{f_{i}\right\}_{i=0}^{s}\right) \in \mathbb{Z}$ tel que pour tout $g \in \varpi^{a} \Xi$ et toute fonction $f \in C_{\mathrm{c}}\left(A / \mathfrak{A}^{v}\right)$, on $a$

$$
\Phi_{H}(f, g)=\sum_{(\alpha, \bar{x})}\left\langle f, \Theta_{\alpha, \bar{x}}\right\rangle \Gamma_{\alpha, \bar{x}}(g)
$$

où $(\alpha, \bar{x})$ parcourt les éléments de $\Pi_{H, n}$.

Démonstration. Pour $t \in F^{\times}$et $f \in C_{\mathrm{c}}^{\infty}(A)$, on note $f_{t} \in C_{\mathrm{c}}^{\infty}(A)$ la fonction définie par $f_{t}(g)=f\left(t^{-1} g\right)$; et pour $T \in \mathscr{D}(A)$, on note $T_{t}$ la distribution sur $A$ définie $\operatorname{par}\left\langle f, T_{t}\right\rangle=\left\langle f_{t}, T\right\rangle$. Soient $\Omega \subset A$ une partie $H$-invariante fermée, et $b \in \mathbb{Z}$. Pour tout $t \in F^{\times}$, l'application $T \mapsto T_{t}$ induit un isomorphisme de $\mathbb{C}$-espaces vectoriels $j^{(1 / d)-b}\left(J_{H}(\Omega)\right) \rightarrow j^{(1 / d)-b-\omega(t)}\left(J_{H}\left(t^{-1} \Omega\right)\right)$. Soit $k \in \mathbb{Z}$. D'après 1.6, il existe un 
entier $b$ telle que pour toute distribution $T \in J_{H}\left(\varpi^{k} \Omega\right)$, il existe des constantes $c_{\alpha, \bar{x}}(T)\left((\alpha, \bar{x}) \in \Pi_{H, n}\right)$ telles que

$$
j^{(1 / d)-b}\left(T-\sum_{(\alpha, \bar{x})} c_{\alpha, \bar{x}}(T) \Theta_{\alpha, \bar{x}}\right)=0
$$

où $(\alpha, \bar{x})$ parcourt les éléments de $\Pi_{H, n}$. Choisissons un élément $t \in F \backslash \mathfrak{p}_{F}$ tel que

$$
\frac{1}{d}-b-\omega(t) \geq v \quad \text { et } \quad\left\{f_{i}\right\}_{i=0}^{s} \subset C_{\mathrm{c}}\left(A / \mathfrak{A}^{(1 / d)-b-\omega(t)}\right) .
$$

Soit $a=k-\omega(t)$. Pour $T \in J_{H}\left(\varpi^{a} \Xi\right)$, posons $\tilde{c}_{\alpha, \bar{x}}(T)=c_{\alpha, \bar{x}}\left(T_{t^{-1}}\right)\left((\alpha, \bar{x}) \in \Pi_{H, n}\right)$. D'après $(*)$, on a

$$
j^{(1 / d)-b-\omega(t)}\left(T-\sum_{(\alpha, \bar{x})} \tilde{c}_{\alpha, \bar{x}}(T) \Theta_{\alpha, \bar{x}}\right)=0
$$

où $(\alpha, \bar{x})$ parcourt les éléments de $\Pi_{H, n}$. Puisque

$$
C_{\mathrm{c}}\left(A / \mathfrak{A}^{(1 / d)-b}\right) \subset C_{\mathrm{c}}\left(A / \mathfrak{A}^{(1 / d)-b-\omega(t)}\right),
$$

pour $T \in J_{H}\left(\varpi^{k} \Omega\right)$, on a $\tilde{c}_{\alpha, \bar{x}}(T)=c_{\alpha, \bar{x}}(T)\left((\alpha, \bar{x}) \in \Pi_{H, n}\right)$; on peut donc poser $c_{\alpha, \bar{x}}(T)=\tilde{c}_{\alpha, \bar{x}}(T)\left(T \in J_{H}\left(\omega^{a} \Xi\right)\right)$. Pour $g \in \varpi^{a} \Xi$, l'intégrale orbitale $\Phi_{H}(\cdot, g)$ est dans $J_{H}\left(\varpi^{a} \Xi\right)$, et l'on pose $c_{\alpha, \bar{x}}(g)=c_{\alpha, \bar{x}}\left(\Phi_{H}(\cdot, g)\right.$ ). D'après (**), pour $g \in \varpi^{a} \Xi$ et $(\alpha, \bar{x}) \in \Pi_{H, n}$, on a $\Phi_{G}\left(f_{i(\alpha, \bar{x})}, g\right)=c_{\alpha, \bar{x}}(g)$; i.e., $\Gamma_{\alpha, \bar{x}}(g)=c_{\alpha, \bar{x}}(g)$. D'où la proposition.

Remarque 2.25. Il résulte de cette démonstration que l'on peut inverser l'ordre des quantificateurs dans la proposition $1.6:$ fixé $v \in \frac{1}{d} \mathbb{Z}$, il existe un entier a tel que pour toute distribution $T \in J_{H}\left(\varpi^{a} \Xi\right)$, il existe des constantes $c_{\alpha, \bar{x}}(T)\left((\alpha, \bar{x}) \in \Pi_{H, n}\right)$ telles que

$$
\mathbf{1}_{\mathfrak{A}^{(1 / d)-v}} \cdot\left(T^{\vee}-\sum_{(\alpha, \bar{x})} c_{\alpha, \bar{x}}(T) \Theta_{\alpha, \bar{x}}^{\vee}\right)=0
$$

où $(\alpha, \bar{x})$ parcourt les éléments de $\Pi_{H, n}$.

Indépendance linéaire des germes $\left.\Gamma_{\alpha, \bar{x}}\right|_{A^{\prime}}\left((\alpha, \bar{x}) \in \Pi_{H, n}\right)$. Pour $y \in A$, on note $d_{y}$ la dimension (en tant que variété $\varpi$-adique) du radical unipotent d'un sousgroupe parabolique $P$ de $G$ associé à $y$ comme dans la démonstration du lemme 2.18. Notons que $P$ n'est pas uniquement determiné par $y$ (alors que $M^{\prime}$ et $P^{\prime \prime}$ le sont); néanmoins $d_{y}$ est bien défini. Soit $t \in F^{\times}$. Pour $(\alpha, \bar{x}) \in \Pi_{H, n}$, on note $\Gamma_{\alpha, \bar{x}}^{t}$ le germe au point 0 dans $A$ défini par $\Gamma_{\alpha, \bar{x}}^{t}(g)=\Gamma_{\alpha, \bar{x}}(t g)$.

Lemme 2.26. Pour $(\alpha, \bar{x}) \in \Pi_{H, n}$, le germe $\Gamma_{\alpha, \bar{x}}$ au point 0 dans $A$ vérifie la formule d'homogénéité

$$
\Gamma_{\alpha, \bar{x}}^{t}(g)=|t|^{d_{g}-(d(\alpha) / 2)} \Gamma_{\alpha, \bar{x}}(g) \quad\left(t \in F^{\times}\right)
$$


avec $d(\alpha)=\operatorname{dim}\left(\mathcal{O}_{\alpha}\right)\left(=\operatorname{dim}\left(\mathbb{O}_{\alpha, \bar{x}}\right)\right)$.

Démonstration. Soit $f \in C_{\mathrm{c}}^{\infty}(A)$. Comme dans [Lemaire 1997, 3.6.1], on montre que (voir la démonstration de 2.23 pour la définition de $f_{t}$ )

$$
\left\langle f_{t}, \Theta_{\alpha, \bar{x}}\right\rangle=|t|^{d(\alpha) / 2}\left\langle f, \Theta_{\alpha, \bar{x}}\right\rangle \quad\left(t \in F^{\times},(\alpha, \bar{x}) \in \Pi_{H, n}\right) .
$$

Et comme dans la démonstration de [Lemaire 1997, 3.6.2], on montre que

$$
\Phi_{H}\left(f_{t}, t g\right)=|t|^{d_{g}} \Phi_{H}(f, g) \quad(g \in A) .
$$

D'après (2.19), pour $t \in F^{\times}$, on a

$$
\left[\Phi_{H}\left(f_{t}, .\right)\right]_{0}=\sum_{(\alpha, \bar{x})}\left\langle f_{t}, \Theta_{\alpha, \bar{x}}\right\rangle \Gamma_{\alpha, \bar{x}}
$$

d'où l'on déduit que

$$
|t|^{d_{g}}\left[\Phi_{H}(f, g)\right]_{0}=\sum_{(\alpha, \bar{x})}|t|^{d(\alpha) / 2}\left\langle f, \Theta_{\alpha, \bar{x}}\right\rangle \Gamma_{\alpha, \bar{x}}^{t}(g) .
$$

Puisque la formule ci-dessus est vraie pour toute fonction $f \in C_{\mathrm{c}}^{\infty}(A)$, d'après la propriété d'unicité de la proposition 2.21, on a

$$
|t|^{-d_{g}+(d(\alpha) / 2)} \Gamma_{\alpha, \bar{x}}^{t}(g)=\Gamma_{\alpha, \bar{x}}(g) \quad\left(t \in F^{\times},(\alpha, \bar{x}) \in \Pi_{H, n}\right) .
$$

D'où le lemme.

Soit $\Phi_{H}: A^{\prime} \times A \rightarrow \mathbb{C}$ la fonction définie par la proposition 2.7

$$
\Phi_{H}\left(y_{1}, y_{2}\right)=\Phi_{H}\left(\cdot, y_{2}\right)^{\vee}\left(y_{1}\right) .
$$

Soit $\left(y_{1}, y_{2}\right) \in A^{\prime} \times A$. D'après 2.6, pour tout sous-groupe ouvert compact $\mathscr{K} \subset H$, on a

$$
\Phi_{H}\left(y_{1}, y_{2}\right)=\Phi_{H}\left(\bar{\Psi}_{A, y_{1}}^{\mathscr{K}}, y_{2}\right)=\int_{H_{y_{2}} \backslash H} \bar{\Psi}_{A, y_{1}}^{\mathscr{Y}}\left(h^{-1} y_{2} h\right) \frac{d h}{d h_{y_{2}}} .
$$

Rappelons (corollaire 2.6) que la restriction de $\bar{\Psi}_{A, y_{1}}^{\mathscr{C}_{\mathrm{C}}} \overline{\mathrm{O}_{H}\left(y_{2}\right)}$ coïncide avec $\left.f\right|_{\overline{\widehat{O}_{H}\left(y_{2}\right)}}$ pour une fonction $f \in C_{\mathrm{c}}^{\infty}(A)$; par conséquent l'intégrale ci-dessus est absolument convergente (lemme 2.18). De plus, toujours d'après 2.6, la fonction $\Phi_{H}$ est localement constante sur $A^{\prime} \times A$.

Proposition 2.27. Soient $v \in \frac{1}{d} \mathbb{Z}$ et $a=a\left(v,\left\{f_{i}\right\}_{i=0}^{s}\right)$ comme dans la proposition 2.23. Alors pour tout $\left(y_{1}, y_{2}\right) \in\left(\mathfrak{A}^{(1 / d)-v} \cap A^{\prime}\right) \times \varpi^{a} \Xi$, on a

$$
\Phi_{H}\left(y_{1}, y_{2}\right)=\sum_{(\alpha, \bar{x})} \Theta_{\alpha, \bar{x}}^{\vee}\left(y_{1}\right) \Gamma_{\alpha, \bar{x}}\left(y_{2}\right)
$$

où $(\alpha, \bar{x})$ parcourt les éléments de $\Pi_{H, n}$. 
Démonstration. D'après 2.23 , pour tous $y_{2} \in \varpi^{a} \Xi$ et $f \in C_{\mathrm{c}}^{\infty}\left(\mathfrak{A}^{(1 / d)-v}\right)$, on a

$$
\Phi_{H}\left(f^{\vee}, y_{2}\right)=\sum_{(\alpha, \bar{x})}\left\langle f, \Theta_{\alpha, \bar{x}}^{\vee}\right\rangle \Gamma_{\alpha, \bar{x}}\left(y_{2}\right)
$$

où $(\alpha, \bar{x})$ parcourt les éléments de $\Pi_{H, n}$. Soit $\left(y_{1}, y_{2}\right) \in\left(\mathfrak{A}^{(1 / d)-v} \cap A^{\prime}\right) \times \varpi^{a} \Xi$. D'après 2.7, il existe un voisinage ouvert compact $\omega$ de $y_{1}$ dans $\mathfrak{A}^{(1 / d)-v} \cap A^{\prime}$ tel que pour tous $y_{1}^{\prime} \in \omega$ et $(\alpha, \bar{x}) \in \Pi_{H, n}$, on a $\phi_{H}\left(\cdot, y_{2}\right)^{\vee}\left(y_{1}^{\prime}\right)=\phi_{H}\left(\cdot, y_{2}\right)^{\vee}\left(y_{1}\right)$ et $\Theta_{\alpha, \bar{x}}^{\vee}\left(y_{1}^{\prime}\right)=\Theta_{\alpha, \bar{x}}^{\vee}\left(y_{1}\right)$. En prenant $f=\operatorname{vol}\left(\omega, d_{A} g\right)^{-1} \mathbf{1}_{\omega}$, on obtient l'égalité cherchée.

Lemme 2.28. Pour $\left(y_{1}, y_{2}\right) \in A_{\mathrm{e}}^{\prime} \times A_{\mathrm{e}}^{\prime}$, on a $\phi_{H}\left(y_{1}, y_{2}\right)=\Phi_{H}\left(y_{2}, y_{1}\right)$.

Démonstration. Soit $\left(y_{1}, y_{2}\right) \in A_{\mathrm{e}}^{\prime} \times A_{\mathrm{e}}^{\prime}$, et soit $\mathscr{K} \subset H$ un sous-groupe ouvert compact. On a

$$
\begin{aligned}
\Phi_{H}\left(y_{1}, y_{2}\right) & =\int_{Z_{H} \backslash H} \bar{\Psi}_{A, y_{1}}^{\mathscr{K}}\left(h^{-1} y_{2} h\right) \frac{d h}{d z} \\
& =\int_{Z_{H} \backslash H}\left(\int_{\mathscr{K}} \bar{\Psi}_{A, y_{1}}^{\mathscr{K}}\left(h^{-1} k y_{2} k^{-1} h\right) d{ }_{\mathscr{K}} k\right) \frac{d h}{d z} \\
& =\int_{Z_{H} \backslash H}\left(\int_{\mathscr{K}} \bar{\Psi}_{A, y_{2}}^{\mathscr{K}}\left(h k y_{1} k^{-1} h^{-1}\right) d_{\mathscr{K}} k\right) \frac{d h}{d z}=\Phi_{H}\left(y_{2}, y_{1}\right) .
\end{aligned}
$$

Lemme 2.29. Il existe un $b=b\left(\left\{f_{i}\right\}_{i=0}^{s}\right) \in \mathbb{Z}$ tel que pour tous $y_{1}, y_{2} \in \mathfrak{A}^{b} \cap A_{\mathrm{e}}^{\prime}$ et tout entier $d \geq 0$, on a

$$
\sum_{(\alpha, \bar{x})} \Theta_{\alpha, \bar{x}}^{\vee}\left(y_{1}\right) \Gamma_{\alpha, \bar{x}}\left(y_{2}\right)=\sum_{(\alpha, \bar{x})} \Theta_{\alpha, \bar{x}}^{\vee}\left(y_{2}\right) \Gamma_{\alpha, \bar{x}}\left(y_{1}\right)
$$

où $(\alpha, \bar{x})$ parcourt les éléments de $\Pi_{H, n}$ tels que $d(\alpha)=d$.

Démonstration. Soit $(\alpha, \bar{x}) \in \Pi_{H, n}$. D'après 1.5 et 2.7 , pour $g \in A^{\prime}$ et $t \in F^{\times}$, on a

$$
\Theta_{\alpha, \bar{x}}^{\vee}(t g)=c \int_{\overline{\bar{O}_{\alpha, \bar{x}}}} \bar{\Psi}_{A, g}^{K_{H}}(t u) d u=|t|^{-\operatorname{dim}\left(\mathfrak{u}_{\alpha}\right)} \Theta_{\alpha, \bar{x}}^{\vee}(g) .
$$

avec $c=c\left(d h_{n_{\alpha}}, d u\right)$. Posons $m=n d$, et soit $\beta \in \Pi_{m}$ la partition définie par

$$
\beta=\left(\alpha_{1}=\cdots=\alpha_{1} \geq \alpha_{2}=\cdots=\alpha_{2} \geq \cdots \geq \alpha_{n}=\cdots=\alpha_{n}\right)
$$

où chaque $\alpha_{i}$ apparaît $d$ fois. D'après [Lemaire 2004, 5.3/1 et 5.3/3], on a $d(\alpha)=$ $2 \sum_{i=1}^{m} i\left(1-\beta_{i}\right)$. Par ailleurs, on a (voire [Lemaire 2004, section 5])

$$
2 \operatorname{dim}\left(\mathfrak{u}_{\alpha}\right)=m^{2}-\operatorname{dim}\left(\mathfrak{m}_{\alpha}\right)=m^{2}-\sum_{i=1}^{m} \hat{\beta}_{i}^{2}
$$


où $\left(\hat{\beta}_{1} \geq \cdots \geq \hat{\beta}_{m}\right) \in \Pi_{m}$ désigne la partition duale de $\beta$, définie par

$$
\hat{\beta}_{i}=\#\left\{k: \beta_{i} \geq k\right\} .
$$

Un calcul facile montre que $m^{2}-\sum_{i=1}^{m} \hat{\beta}_{i}^{2}=2 \sum_{i=1}^{m} i\left(1-\beta_{i}\right)$. On a donc $\operatorname{dim} \mathfrak{u}_{\alpha}=$ $\frac{1}{2} d(\alpha)$, d'où la formule d'homogénéité

$$
\Theta_{\alpha, \bar{x}}^{\vee}(t g)=|t|^{-\frac{d(\alpha)}{2}} \Theta_{\alpha, \bar{x}}^{\vee}(g) \quad\left(g \in A^{\prime}, t \in F^{\times}\right) .
$$

Soient $v \in \frac{1}{d} \mathbb{Z}$ et $a=a\left(v,\left\{f_{i}\right\}_{i=0}^{s}\right)$ comme dans la proposition 2.23. Choisissons un $b \in \mathbb{Z}$ tel que $b \geq \frac{1}{d}-v$ et $\mathfrak{A}^{b} \subset \varpi^{a} \Xi$. D'après 2.27 et 2.28 , pour tous $y_{1}, y_{2} \in$ $\mathfrak{A}^{b} \cap A_{\mathrm{e}}^{\prime}$, on a

$$
\sum_{(\alpha, \bar{x})} \Theta_{\alpha, \bar{x}}^{\vee}\left(y_{1}\right) \Gamma_{\alpha, \bar{x}}\left(y_{2}\right)=\sum_{(\alpha, \bar{x})} \Theta_{\alpha, \bar{x}}^{\vee}\left(y_{2}\right) \Gamma_{\alpha, \bar{x}}\left(y_{1}\right)
$$

où $(\alpha, \bar{x})$ parcourt les éléments de $\Pi_{H, n}$. Puisque pour $y \in A_{\mathrm{e}}^{\prime}$, on a $d_{y}=0$, l'égalité ci-dessus jointe aux formules d'homogénéité 2.26 et (2.30), impliquent le lemme (fixer $y_{1}, y_{2}$ comme ci-dessus, puis remplacer $y_{2}$ par $t y_{2}$ pour $t \in \mathfrak{o}_{F} \backslash\{0\}$ ).

Pour $(\alpha, \bar{x}) \in \Pi_{H, n}$, on note $\Gamma_{\alpha, \bar{x}}^{\prime}$ et $\Gamma_{\mathrm{e} ; \alpha, \bar{x}}^{\prime}$ les germes de fonctions au point 0 respectivement dans $A^{\prime}$ et dans $A_{\mathrm{e}}^{\prime}$ [Lemaire 2004, 3.5] définis par $\Gamma_{\alpha, \bar{x}}^{\prime}=\left.\Gamma_{\alpha, \bar{x}}\right|_{A^{\prime}}$ et $\Gamma_{\mathrm{e} ; \alpha, \bar{x}}^{\prime}=\left.\Gamma_{\alpha, \bar{x}}\right|_{A_{\mathrm{e}}^{\prime}}$; et l'on pose $\Gamma_{\mathrm{e}, i(\alpha, \bar{x})}^{\prime}=\Gamma_{\mathrm{e} ; \alpha, \bar{x}}^{\prime}$.

Lemme 2.31. Il existe une constante $c_{0} \neq 0$ telle que $\Gamma_{\mathrm{e}, 0}^{\prime}=c_{0}$.

Démonstration. Soit $\left.b=b\left(\left\{f_{i}\right\}_{i=0}^{s}\right\}\right)$ comme dans le lemme 2.29. Puisque $\Theta_{(1, \ldots, 1), 1}^{\vee}$ est une mesure de Haar sur $A$, en prenant $d=0$ dans 2.29, on obtient

$$
\Gamma_{(1, \ldots, 1), 1}\left(y_{1}\right)=\Gamma_{(1, \ldots, 1), 1}\left(y_{2}\right) \quad\left(y_{1}, y_{2} \in \mathfrak{A}^{b} \cap A_{\mathrm{e}}^{\prime}\right) ;
$$

i.e., $\Gamma_{\mathrm{e}, 0}^{\prime}=c$ pour une constante $c \in \mathbb{C}$. Il s'agit de montrer que cette constante est non nulle. D'après la remarque 2.22 , dont on reprend ici les notations, on a $\Gamma_{(1, \ldots, 1), 1}=\Gamma_{(1, \ldots, 1)}$. On peut donc supposer que $H=G$. Si $D=F$ (i.e., si $G \simeq$ $\mathrm{GL}_{n}(F)$ ), la non-nullité de $c$ est démontrée par Henniart dans l'appendice 3 de [Henniart 1984] (les germes associés aux $G$-orbites unipotentes de $G$ sont donnés par $\Gamma_{\alpha}(\cdot-1)$ pour $\left.\alpha \in \Pi_{n}\right)$. Sa démonstration reste valable pour $D \neq F$.

Proposition 2.32. Les germes $\Gamma_{\alpha, \bar{x}}^{\prime}\left((\alpha, \bar{x}) \in \Pi_{H, n}\right)$ sont $\mathbb{C}$-linéairement indépendants.

Démonstration. Si $[\phi]_{0}$ est un germe de fonctions en 0 , on pose $[\phi]_{0}^{\prime}=\left.[\phi]_{0}\right|_{A^{\prime}}$ et $[\phi]_{0, \mathrm{e}}^{\prime}=\left.[\phi]_{0}\right|_{A_{\mathrm{e}}^{\prime}}$. Soit $\left\{a_{\alpha, \bar{x}}:(\alpha, \bar{x}) \in \Pi_{H, n}\right\} \subset \mathbb{C}$ une famille telle que

$$
\sum_{(\alpha, \bar{x})} a_{\alpha, \bar{x}} \Gamma_{\alpha, \bar{x}}^{\prime}=0
$$


où $(\alpha, \bar{x})$ parcourt les éléments de $\Pi_{H, n}$. Posons $a_{i(\alpha, \bar{x})}=a_{\alpha, \bar{x}}\left((\alpha, \bar{x}) \in \Pi_{H, n}\right)$, et notons $f \in C_{\mathrm{c}}^{\infty}(A)$ la fonction définie par $f=\sum_{i=0}^{s} a_{i} f_{i}$. D'après 2.21, on a $\left[\Phi_{H}(f, \cdot)\right]_{0}^{\prime}$.

Posons $Q(g)=\sum_{i=1}^{s} a_{i} \Phi_{H}\left(f_{i}, g\right)(g \in A)$. D'après le lemme 2.26, pour $t \in$ $\mathfrak{o}_{F} \backslash\{0\}$, on a

$$
[Q(t \cdot)]_{0, \mathrm{e}}^{\prime}=\sum_{i=1}^{s} a_{i}|t|^{-d(i) / 2}\left[\Phi_{H}\left(f_{i}, \cdot\right)\right]_{0, \mathrm{e}}^{\prime}
$$

avec $d(i(\alpha, \bar{x}))=d(\alpha)\left((\alpha, \bar{x}) \in \Pi_{H, n}\right)$. On en déduit que si le germe $[Q]_{0, \mathrm{e}}^{\prime}$ n'est pas nul, alors il existe un $\gamma \in A_{\mathrm{e}}^{\prime}$ tel que $\lim _{t \rightarrow 0, t \neq 0}|Q(t \gamma)|=+\infty$; ce qui est impossible puisque, par la proposition 2.21,

$$
0=\left[\Phi_{H}(f, \cdot)\right]_{0, \mathrm{e}}^{\prime}=a_{0}\left[\Phi_{H}\left(f_{0}, \cdot\right)\right]_{0, \mathrm{e}}^{\prime}+[Q]_{0, \mathrm{e}}^{\prime}
$$

et que le germe $\left[\Phi_{H}\left(f_{0}, \cdot\right)\right]_{0, \mathrm{e}}^{\prime}$ est constant (lemme 2.31). Par conséquent $[Q]_{0, \mathrm{e}}^{\prime}=0$ et $a_{0}\left[\Phi_{H}\left(f_{0}, \cdot\right)\right]_{0, \mathrm{e}}^{\prime}=0$; d'où $a_{0}\left(=\Phi_{H}(f, 0)\right)=0$ (encore 2.31).

Fixons un $i \in\{1, \ldots, s\}$ et montrons que $a_{i}=0$. Soit $(\alpha, \bar{x}) \in \Pi_{H, n}$ l'élément tel que $i(\alpha, \bar{x})=i$. D'après (2.19), il existe une (unique) constante $c_{i}>0$ telle que pour toute fonction $f^{\prime} \in C_{\mathrm{c}}^{\infty}(A)$, on a la formule de descente

$$
\Phi_{H}\left(f^{\prime}, n_{\alpha, x}\right)=c_{i} f_{H, \mathfrak{p}_{\alpha}}^{\prime}(0) .
$$

Par ailleurs, d'après (2.10), on a $\left.\left[\Phi_{M_{H, \alpha}}\left(f_{H, \mathfrak{p}_{\alpha}}, \cdot\right)\right]_{0}\right|_{\mathfrak{m}_{\alpha} \cap A^{\prime}}=0$. Comme tout voisinage de 0 dans $\mathfrak{m}_{\alpha}$ rencontre $\mathfrak{m}_{\alpha, \mathrm{e}}^{\prime}$, en remplaçant $H$ par $M_{H, \alpha}$ et $f$ par $f_{H, \mathfrak{p}_{\alpha}}$ dans le raisonnement précédent, on obtient que $f_{H, \mathfrak{p}_{\alpha}}(0)=0$. Donc $a_{i}\left(=\Phi_{H}\left(f, n_{\alpha, x}\right)\right)=0$. Ce qui achève la démonstration de la proposition.

Proposition 2.33. Soit $T \in J_{H}^{*}(A)$. Alors la transformée de Fourier $T^{\vee}$ est intégrable au voisinage de 0 dans $A$.

Démonstration. Soit $v \in \frac{1}{d} \mathbb{Z}$, et soit un entier $a$ tel que :

- pour tout $g \in \varpi^{a} \Xi$, on a

$$
j^{v}\left(\Phi_{H}(\cdot, g)-\sum_{(\alpha, \bar{x})} \Gamma_{\alpha, \bar{x}}(g) \Theta_{\alpha, \bar{x}}\right)=0
$$

où $(\alpha, \bar{x})$ parcourt les éléments de $\Pi_{H, n}$;

- pour toute distribution $T \in J_{H}\left(\varpi^{a} \Xi\right)$, il existe des constantes $c_{\alpha, \bar{x}}(T)((\alpha, \bar{x}) \in$ $\left.\Pi_{H, n}\right)$ telles que

$$
j^{v}\left(T-\sum_{(\alpha, \bar{x})} c_{\alpha, \bar{x}}(T) \Theta_{\alpha, \bar{x}}\right)=0
$$

où $(\alpha, \bar{x})$ parcourt les éléments de $\Pi_{H, n}$. 
D'après 2.23 et 2.25 , un tel $a$ existe. Posons $\mathscr{C}=C_{\mathrm{c}}\left(A / \mathfrak{A}^{v}\right)$ et soit $\mathscr{C}_{0} \subset \mathscr{C}$ le sous-espace vectoriel formé des fonctions $f$ telles que $\Phi_{G}(f, \gamma)=0$ pour tout $\gamma \in \varpi^{a} \Xi \cap A^{\prime}$. Si $f \in \mathscr{C}_{0}$, alors (proposition 2.32) $\left\langle f, \Theta_{\alpha, \bar{x}}\right\rangle=0$ pour tout $(\alpha, \bar{x}) \in$ $\Pi_{H, n}$, donc $\langle f, T\rangle=0$ pour tout $T \in J_{H}\left(\varpi^{a} \Xi\right)$. Par conséquent, la forme bilinéaire $\mathscr{C} \times J_{H}\left(\varpi^{a} \Xi\right) \rightarrow \mathbb{C},(f, T) \mapsto\langle f, T\rangle$ induit une forme bilinéaire non dégénérée $\mathscr{C} / \mathscr{C}_{0} \times j^{v}\left(J_{H}\left(\varpi^{a} \Xi\right)\right) \rightarrow \mathbb{C}$. Puisque $\operatorname{dim}_{\mathbb{C}}\left(j^{v}\left(J_{H}\left(\varpi^{a} \Xi\right)\right)\right)<+\infty$, on en déduit qu'il existe un ensemble fini $\left\{\gamma_{1}, \ldots, \gamma_{r}\right\} \subset \varpi^{a} \Xi \cap A^{\prime}$ tel que $j^{v}\left(J_{H}\left(\varpi^{a} \Xi\right)\right)=$ $\left\langle j^{v}\left(\Phi_{H}\left(\cdot, \gamma_{i}\right)\right): i=1, \ldots, r\right\rangle$. Posons $\mu=\frac{1}{d}-v$. Par dualité, on obtient que pour toute distribution $T \in J_{H}\left(\varpi^{a} \Xi\right)$, la restriction $\mathbf{1}_{\mathfrak{A}^{\mu}} \cdot T^{\vee}$ est combinaison linéaire des restrictions $\mathbf{1}_{\mathfrak{A}^{\mu}} \cdot \Phi_{H}\left(\cdot, \gamma_{i}\right)^{\vee}$ pour $i=1, \ldots, r$. On conclut grâce au corollaire 2.17 et au lemme 2.13 .

Corollaire 2.34. Pour $y \in A$, la transformée de Fourier $\Phi_{H}(\cdot, y)^{\vee}$ est intégrable au voisinage de 0 dans $A$.

Démonstration. Pour $y \in A, \overline{\widehat{O}_{H}(y)}$ est compact modulo $H$-conjugaison : en effet, il existe un $b \in \mathbb{Z}$ tel que $y \in \varpi^{b} \Xi$ où $\Xi$ est la partie $G$-invariante ouverte et fermée dans $A$ définie en [Lemaire 2004, 1.2]. Puisque $\varpi^{b} \Xi \subset{ }^{G}\left(\mathfrak{A}^{b-1}\right)$ (ibidem), $\varpi^{b} \Xi$ est compact modulo $G$-conjugaison, donc est compact modulo $H$-conjugaison.

\section{Intégrabilité locale des caractères de $H$}

Descente des distributions $H$-invariantes au voisinage d'un élément pur. On reprend en la modifiant la construction de [Lemaire 2004, 2] ( $E=F, \sigma=1)$. Soit $x \in H$ un élément $G$-pur. Le centralisateur $B=\{g \in A: g x-x g=0\}$ de $x$ dans $A$, est une $F$-algèbre simple. Soient $F^{\prime}$ et $G_{x}=B^{\times} \subset G$ respectivement le centre et le groupe multiplicatif de $B$ (notons, pour éviter les confusions, que dans [Lemaire 2004] le groupe $G_{x}$ est noté $H$ ). On pose $H_{x}=G_{x} \cap H$. Fixons un caractère additif $\Psi_{F^{\prime}}$ de $F^{\prime}$ de conducteur $\mathfrak{p}_{F^{\prime}}$ et posons $\Psi_{B}=\Psi_{F^{\prime}}$ otr $\operatorname{tr}_{B / F^{\prime}}$ où $\operatorname{tr}_{B / F^{\prime}}^{\prime}: B \rightarrow F^{\prime}$ désigne la trace réduite. Soit $s: A \rightarrow B$ l'homomorphisme surjectif de $(B, B)$-bimodules défini par $\Psi_{B}(s(y) b)=\Psi_{A}(y b)$ pour tout $(y, b) \in A \times B$. Soit $\varrho \in G$ tel que $s(\varrho)=1$, et soit $W$ un sous- $F$-espace vectoriel de $A$ tel que $A=W \oplus B$. Soit $C$ l'image de l'application $F$-linéaire $A \rightarrow A, y \mapsto x y x^{-1}-y$. Pour $u \in B$, on note $\Phi_{u}: W \times B \rightarrow A$ l'application $F$-linéaire définie par

$$
\Phi_{u}(v, b)=(1+u \varrho) x v-v(1+u \varrho) x+b \varrho x .
$$

Puisque $A=C \oplus B \varrho$, l'application $\Phi_{0}$ est bijective. Soit $\mathfrak{U}$ le voisinage ouvert de 0 dans $B$ formé des $u \in B$ vérifiant les conditions

$$
(1+u \varrho) \in H \quad \text { et } \quad \operatorname{det}_{F}\left(\Phi_{u} \circ \Phi_{0}^{-1} ; A\right) \neq 0 .
$$


Pour $b \in H_{x}$, on pose $\mathfrak{U}_{b}=b^{-1} \mathfrak{U} b$. D'après [Lemaire 2004, 2.3], pour $b \in H_{x}$, l'application $\zeta_{b}: H \times \mathfrak{U}_{b} \rightarrow H,(h, u) \mapsto h^{-1}\left(1+u b^{-1} \varrho b\right) x h$ est partout submersive. Soit $\mathfrak{O} \subset \mathfrak{U}$ un voisinage ouvert compact de 0 dans $B$, et soit $\Xi_{\natural}$ la partie $G_{x}$-invariante ouverte et fermée dans $B$ définie comme en [Lemaire 2004, 1.2] (en remplaçant $A$ par $B$ et $F$ par $F^{\prime}$ ). Soit $\varpi^{\prime}$ une uniformisante de $F^{\prime}$. Puisque $H_{x}$ est ouvert et distingué dans $G_{x},\left\langle\varpi^{\prime}\right\rangle H_{x}$ est un sous-groupe d'indice fini de $G_{x}$. Par conséquent, $\Xi_{\natural}$ est compact modulo $H_{x}$-conjugaison.

Soit $J_{H}(H) \subset \mathscr{D}(H)$ l'espace des distributions $H$-invariantes sur $H$ (pour l'action de $H$ par conjugaison). Soit $d_{B} u$ la mesure de Haar sur $B$ normalisée par un (i.e., par tout) $\mathfrak{o}_{F^{\prime}}$-ordre maximal dans $B$. Pour $T \in J_{H}(H)$ et $k \in \mathbb{Z}$ tel que $\varpi^{\prime k} \Xi_{\natural} \subset H_{x} \mathfrak{O}$, on note $T_{\natural, k}$ la distribution sur $B$ à support dans $\varpi^{\prime k} \Xi_{\natural}$ définie grâce à la famille de submersion $\left\{\zeta_{b}\right\}_{b \in H_{x}}$ (et via le choix des mesures de Haar $d h$ et $d_{B} u$ respectivement sur $H$ et $B$ ) comme en [Lemaire 2004, 2].

Soit $\mathscr{K}$ un sous-groupe ouvert compact de $H$. Si $\rho \in \epsilon(\mathscr{K})$, on note $\chi_{\rho}$ le caractère $k \mapsto$ trace $\rho(k), \operatorname{deg}(\rho)$ le degré de $\rho$, et l'on pose

$$
\xi_{\rho}=\frac{\operatorname{deg}(\rho)}{\operatorname{vol}(\mathscr{K}, d h)} \chi_{\rho} .
$$

Proposition 3.1 [Lemaire 2004, 2.7]. Soient $T \in J_{H}(H)$ et $k \in \mathbb{Z}$ tel que $\varpi^{\prime k} \Xi_{\natural} \subset$ $H_{x} \mathfrak{O}$.

(1) La distribution $T_{\natural, k} \in \mathscr{D}(B)$ est $H_{x}$-invariante.

(2) Soit $\mathscr{K}$ un sous-groupe ouvert compact de H. Pour $f \in C_{\mathrm{c}}^{\infty}\left(\varpi^{\prime k} \Xi_{\natural} \cap \mathfrak{O}\right)$, on a

$$
\left\langle f, T_{\natural, k}\right\rangle=\sum_{\rho \in \in(\mathscr{K})} \int_{\mathfrak{O}} f(u) T * \xi_{\rho}((1+u \varrho) x) d_{B} u .
$$

Corollaire 3.2 [Lemaire 2004, 2.8]. Soient $\mathfrak{B}$ un sous-groupe ouvert compact de $B$ tel que $\mathfrak{B} \subset \varpi^{\prime k} \Xi_{\natural} \cap \mathfrak{O}$ et $(1+\mathfrak{B} \varrho) \subset \mathscr{K}$, et $\chi \in \epsilon(\mathfrak{B})$ un caractère tel que $\left\langle\chi, T_{\natural, k}\right\rangle \neq 0$. Alors il existe une représentation $\rho \in \epsilon(\mathscr{K})$ telle que

$$
\left.T * \xi_{\rho}\right|_{\mathscr{L} x} \neq 0 \quad \text { et } \quad \int_{\mathfrak{B}} \chi(u) \rho(1+u \varrho) d_{B} u \neq 0 .
$$

Étude locale des caractères de $H$. Pour $v \in\left(\frac{1}{d} \mathbb{Z}\right)_{>0}$, notons $e_{v}: \mathfrak{A}^{v} \rightarrow K^{v}$ la bijection $y \mapsto 1+y$. Pour $v, \mu \in\left(\frac{1}{d} \mathbb{Z}\right)_{>0}$ tels que $\mu \geq 2 v$, le groupe $K^{\mu-v} / K^{\mu}$ est central dans $K^{v} / K^{\mu}$, et l'application $e_{\mu-v}$ induit par passage aux quotients un isomorphisme de groupes $\mathfrak{A}^{\mu-v} / \mathfrak{A}^{\mu} \rightarrow K^{\mu-v} / K^{\mu}$. Pour $g \in A$, on note $\Psi_{g}$ le caractère de $A$ donné par $\Psi_{g}(y)=\Psi_{A}(g y)$. L'application $g \mapsto \Psi_{g}$ identifie $A$ a son dual de Pontryagin $\epsilon(A)$, et pour $v, \mu \in \mathbb{Z}_{>0}$ tels que $\mu \geq 2 v$, elle induit une identification $\mathfrak{A}^{(1 / d)-\mu} / \mathfrak{A}^{(1 / d)+\nu-\mu}=\epsilon\left(\mathfrak{A}^{\mu-v} / \mathfrak{A}^{\mu}\right)$; d'où une identification

$$
\mathfrak{A}^{(1 / d)-\mu} / \mathfrak{A}^{(1 / d)+v-\mu}=\epsilon\left(K^{\mu-v} / K^{\mu}\right) .
$$


Si $\rho \in \epsilon\left(K^{a}\right)$ pour un entier $a>0$, on note $n(\rho)$ le plus petit entier $b \geq a$ tel que $\left.\rho\right|_{K^{b}}=1$. Pour $a, b \in \mathbb{Z}_{>0}$ tels que $b \geq a$, on note $\epsilon_{b}\left(K^{a}\right) \subset \epsilon\left(K^{a}\right)$ le sous-ensemble formé des représentations $\rho$ telles que $n(\rho)=b$. Pour $a>0$ et $\rho \in \epsilon\left(K^{a}\right)$ tels que $b=n(\rho) \geq 2 a$, le caractère $\omega_{\rho} \in \epsilon\left(K^{b-a} / K^{b}\right)$ donné par $\left.\rho\right|_{K^{b-a}}=\omega_{\rho} \cdot \operatorname{Id}_{K^{b-a}}$ définit comme en [Lemaire 2004] 3 un élément $Z_{\rho} \in \mathfrak{A}^{(1 / d)-b} / \mathfrak{A}^{(1 / d)+a-b}$. Mutatis mutandis, les lemmes 3.1 et 3.2 de [Lemaire 2004] sont vrais ici.

Pour $T \in \mathscr{D}(H)$ et $f \in C_{\mathrm{c}}^{\infty}(H)$, on note $T * f$ la fonction sur $H$ définie par $T * f(y)=\int_{H}\left(y h^{-1}\right) d T h$.

Remarque 3.3 (avec les notations de [Lemaire 2004]). La proposition 3.4 de [Lemaire 2004] est vraie pour toute distribution $T \in J_{\sigma}\left(G_{E}\right)$, et pas seulement pour le caractère tordu $\Theta_{\pi, \sigma}$ d'une représentation $\pi \in \tilde{\epsilon}_{\sigma}\left(G_{E}\right)$.

Proposition 3.4 [Lemaire 2004, 3.4]. Il existe un entier $d_{0}=d_{0}(x, \mathfrak{A}) \leq 0$ tel que pour tous $a \in \mathbb{Z}_{>0}$ tel que $K^{a} \subset H, b \in \mathbb{Z}_{\geq 2 a}, T \in J_{H}(H)$ et $\rho \in \epsilon_{b}\left(K^{a}\right)$ tels que $\left.T * \xi_{\rho}\right|_{K^{a} x} \neq 0$, on a l'inclusion $Z_{\rho} \subset B+\mathfrak{A}^{a-b+d_{0}}$.

Si $\pi$ est une représentation complexe lisse irréductible de $H$, le groupe $Z \cap H$ opère sur l'espace de $\pi$ via un caractère (lisse), et toute extension de ce caractère en un caractère lisse de $Z$ permet d'étendre $\pi$ en une représentation lisse (irréductible) de $Z H$. Puisque $Z H$ est d'indice fini dans $G$ et que toute représentation complexe lisse irréductible de $G$ est admissible, on en déduit (voir [Henniart 2001, 2.2]) que toute représentation complexe lisse irréductible de $H$ est admissible. Pour toute représentation complexe lisse admissible $\pi$ de $H$, on note $\Theta_{\pi} \in J_{H}(H)$ la distribution trace $(\pi d h)$; cette distribution ne dépend pas vraiment de $\pi$ mais seulement de sa classe d'équivalence.

Proposition 3.5 [Lemaire 2004, 3.3]. Soient $a, b \in \mathbb{Z}_{>0}$ tels que $b \geq 2 a$ et $K^{a} \subset H$. Soient $\pi \in \epsilon(H)$ et $\rho \in \epsilon_{b}\left(K^{a}\right)$ tels que $\pi^{K^{a}} \neq 0$ et $\Theta_{\pi} * \xi_{\rho} \neq 0$. Alors on $a$ $Z_{\rho} \cap \mathfrak{N} \neq \varnothing$.

Démonstration. Elle est identique à celle de [Lemaire 2004, 3.3]. Un mot sur la décomposition de Cartan de $g_{i}$ qu'on y a utilisée : soit $W$ un sous-espace non nul de l'espace de $\pi$ tel que $\pi\left(K^{a}\right)(W)=W$ et $\left.\pi\right|_{K^{a}}$ induise sur $W$ une représentation équivalente à $\rho$, et soit $w \in W \backslash\{0\}$. Alors il existe un $h \in H$ tel que $\pi\left(\mathbf{1}_{K^{a, h}}\right)(w) \neq 0$ avec $K^{a h}=h^{-1} K^{a} h$. En écrivant une décomposition de Cartan de $h$ dans $G$, on obtient que $\left.\omega_{\rho}\right|_{\left(K^{b-a} \cap P_{0}^{\prime}\right) K^{b}}=1$ pour un sous-groupe parabolique minimal $P_{0}^{\prime}$ de $G$.

Étude locale des réductions à $B$ des caractères de $H$. Soit $W$ un $B$-module simple (à gauche). Alors $\Delta^{o}=\operatorname{End}_{B}(W)$ est une algèbre à division de centre $F^{\prime}$, et $B=$ $\operatorname{End}_{\Delta}(W)$ où $\Delta$ désigne l'algèbre opposée à $\Delta^{o}$. Soit $\delta^{\prime}=e\left(F^{\prime} / F\right)\left(\Delta: F^{\prime}\right)^{1 / 2}$ où $e^{\prime}\left(F^{\prime} / F\right)$ désigne l'indice de ramification de l'extension $F^{\prime} / F$. Fixons un $\mathfrak{o}_{\Delta^{-}}$ réseau $\Lambda_{\natural}$ dans $W$ et posons $\mathfrak{A}_{\natural}^{v}=\operatorname{Hom}_{\mathfrak{o}_{\Delta}}\left(\Lambda_{\natural}, \Lambda_{\natural} \cdot \mathfrak{p}_{\Delta}^{\delta^{\prime} v}\right)\left(v \in \frac{1}{\delta^{\prime}} \mathbb{Z}\right)$. On note $f \mapsto f^{\vee}$ 
(resp. $T \mapsto T^{\vee}$ ) la transformée de Fourier sur $C_{\mathrm{c}}^{\infty}(B)$ (resp. sur $\mathscr{D}(B)$ ) définie par $f^{\vee}(v)=\int_{B} f(u) \overline{\Psi_{B}(v u)} d_{B}(u)\left(\operatorname{resp} .\left\langle f, T^{\vee}\right\rangle=\left\langle f^{\vee}, T\right\rangle\right)$.

Soit $a_{0}$ le plus petit entier $i$ tel que $\mathfrak{A}_{\natural}^{i} \varrho \subset \mathfrak{A}$. On a $\mathfrak{A}_{\natural}^{a+a_{0}} \varrho \subset \mathfrak{A}^{a}(a \in \mathbb{Z})$. Fixons un entier $k_{0}$ tel que $\varpi^{\prime k_{0}} \Xi_{\natural} \subset H_{x} \mathfrak{O}$, et pour $\pi \in \epsilon(H)$, notons $\theta_{\pi}$ la distribution $H_{x}$-invariante sur $B$ définie par $\theta_{\pi}=\left(\Theta_{\pi}\right)_{\natural, k_{0}}$ (voir proposition 3.1). On note $\mathfrak{N}_{\natural}$ l'ensemble $\mathfrak{N} \cap B$ des éléments nilpotents de $B$. Soit $q$ le cardinal du corps résiduel de $D$, et soit $d_{\mathfrak{A}}$ la distance sur $A$ définie par $d_{\mathfrak{A}}(y, z)=q^{-d \omega_{\mathfrak{A}}(z-y)}(y, z \in A)$; voir [Lemaire 2004,1$]$ pour la définition de la valuation $\omega_{\mathfrak{A}}: A \rightarrow \frac{1}{d} \mathbb{Z} \cup\{\infty\}$. On pose $\mathfrak{N}^{*}=\mathfrak{N} \cap\left(\mathfrak{A} \backslash \mathfrak{A}^{1}\right)$.

Lemme 3.6. Pour tout voisinage ouvert compact $\mathscr{q}^{*}$ de $\mathfrak{N}^{*} \cap B$ dans $\left(\mathfrak{A} \backslash \mathfrak{A}^{1}\right) \cap B$, il existe un entier $v=v\left(s, \mathscr{V}^{*}\right)$ tel que pour tout $g \in \mathfrak{N}^{*}$, on a l'implication $d_{\mathfrak{A}}(g, B) \leq$ $q^{-v} \Rightarrow s(\varrho g) \in \mathscr{V}^{*}$.

Proposition 3.7 [Lemaire 2004, 4.2]. Soit $\mathscr{V}$ un voisinage ouvert et fermé de $\mathfrak{N}_{\natural} \backslash$ $\{0\}$ dans $B \backslash\{0\}$ tel que $F^{\times \mathscr{V}}=\mathscr{V}$, et soit $\pi \in \epsilon(H)$. Il existe un entier a $>0$ tel que pour tout $\chi \in \epsilon\left(\mathfrak{A}_{\natural}^{a+a_{0}}\right)$ tel que $\left\langle\bar{\chi}, \theta_{\pi}\right\rangle \neq 0$, on a l'inclusion $\operatorname{Supp}\left(\chi^{\vee}\right) \subset \mathcal{V} \cup \mathfrak{A}_{\natural}^{\mu}$ avec $\mu=\delta^{\prime-1}-2 a-a_{0}+1$.

Démonstration. Posons $\mathscr{V}^{*}=\mathscr{V} \cap\left(\mathfrak{A} \backslash \mathfrak{A}^{1}\right)$. Soient $d_{0}=d_{0}(x, \mathfrak{A}) \leq 0$ et $v=v\left(s, \mathscr{V}^{*}\right)$ des entiers comme dans la proposition 3.4 et le lemme 3.6. Soit un entier $a>0$ tel que :

(a) $\mathfrak{A}_{\natural}^{a+a_{0}} \subset \omega^{\prime k_{0}} \Xi_{\natural} \cap \mathfrak{O}$,

(b) $a \geq \frac{1}{d} v-d_{0}+\left[\frac{1}{d}\right]$ où [ ] désigne la partie entière,

(c) $\mathscr{V}^{*}+\mathfrak{A}_{\natural}^{\delta^{\prime-1}+a-a_{0}}=\mathscr{V}^{*}$,

(d) $K^{a} \subset H$ et $\pi^{K^{a}} \neq 0$.

D'après la démonstration de [Lemaire 2004, 4.2], cet entier $a$ convient.

Soit $d h_{\natural}$ une mesure de Haar sur $H_{x}$. Fixons un système de représentants $\mathcal{N}_{H, \natural}$ dans $\mathfrak{N}_{\natural}$ des $H_{x}$-orbites nilpotentes de $B$ (cf. la section 1 ; on rappelle que $H_{x}$ est un sous-groupe ouvert distingué de $\left.G_{x}=B^{\times}\right)$. Pour chaque $n \in \mathcal{N}_{H, \downarrow}$, choisissons une mesure de Haar $d h_{\natural, n}$ sur le centralisateur $\left(H_{x}\right)_{n}$ de $n$ dans $H_{x}$, et notons $\Theta_{\natural, n}$ la distribution sur $B$ définie par la proposition 1.5 :

$$
\left\langle f, \Theta_{\natural, n}\right\rangle=\int_{\left(H_{x}\right)_{n} \backslash H_{x}} f\left(h_{\natural}^{-1} n h_{\natural}\right) \frac{d h_{\natural}}{d h_{\natural, n}} .
$$

D'après le corollaire 2.34 et la proposition 2.7, pour $n \in \mathcal{N}_{H, \downarrow}$, la transformée de Fourier $\Theta_{\natural, n}^{\vee}$ est une distribution intégrable au voisinage de 0 dans $B$, localement constante sur l'ensemble $B^{\prime}$ des éléments semisimples réguliers de $B$. 
Proposition 3.8 [Lemaire 2004, 4.3]. Soit $\pi \in \epsilon(H)$. La distribution $\theta_{\pi}$ est intégrable au voisinage de 0 dans $B$. Plus précisément, il existe un entier $m$ et des constantes $c_{n}(\pi)\left(n \in \mathcal{N}_{H, \sharp}\right)$ tels que

$$
\mathbf{1}_{\mathfrak{A}_{\sharp}^{m}} \cdot\left(\theta_{\pi}-\sum_{n \in \mathcal{N}_{H, \sharp}} c_{n}(\pi) \Theta_{\natural, n}^{\vee}\right)=0 .
$$

Corollaire 3.9 [Lemaire 2004, 4.4]. La distribution $\Theta_{\pi}$ est intégrable au voisinage de $x$ dans $H$. Plus précisément, il existe un entier $m>a_{0}$ tel que $K^{m-a_{0}} \subset \mathrm{H}$ et

$$
\Theta_{\pi}((1+u \varrho) x)=\sum_{n \in \mathcal{N}_{H, \natural}} c_{n}(\pi) \Theta_{\natural, n}^{\vee}(u)
$$

pour presque tout $u \in \mathfrak{A}_{\natural}^{m}$ (resp. pour tout $u \in \mathfrak{A}_{\natural}^{m} \cap B^{\prime}$ ).

Réduction aux éléments $G$-purs de $H$ (descente parabolique). Soit $g \in A$, et posons $\Delta_{g}=\operatorname{det}^{\prime}\left(G_{g}\right)$. Le groupe $H G_{g}$ est distingué dans $G$, et l'application det' induit par passages aux quotients un isomorphisme de groupes $\delta_{g}: H G_{g} \backslash G \rightarrow$ $\Delta_{H} \Delta_{g} \backslash F^{\times}$. Puisque $H G_{g} \supset{ }_{\varpi} H$, le groupe $H G_{g} \backslash G$ est fini. La $G$-orbite $\mathscr{O}_{g}=$ $0_{G}(g)$ est un $H$-ensemble (pour l'opération de $H$ par conjugaison), et via $\delta_{g}$, l'ensemble des $H$-orbites de $\mathcal{O}_{g}$ est un torseur sous $\Delta_{H} \Delta_{g} \backslash F^{\times}$. Pour $\bar{y} \in \Delta_{H} \Delta_{g} \backslash F^{\times}$, choisissons un représentant $y$ de $\delta_{g}^{-1}(\bar{y})$ dans $G$, et posons $\bigcirc_{g, \bar{x}}=\left\{h^{-1} y^{-1} g y h\right.$ : $h \in H\}$. On a $O_{g}=\bigsqcup_{\bar{y}} O_{g, \bar{y}}$ où $\bar{y}$ parcourt les éléments de $\Delta_{H} \Delta_{g} \backslash F^{\times}$, et chaque $H$-orbite $\hat{O}_{g}, \bar{y}$ est ouverte et fermée dans $\mathrm{O}_{g}$.

D'après [Lemaire 2004, 5.2/2] et le paragraphe ci-dessus, $\overline{\mathrm{O}_{H}(g)}$ est union de $0_{H}(g)$ et d'un nombre fini de $H$-orbites de dimension (en tant que variétés $\varpi-$ adiques) strictement inférieure à celle de $\widehat{O}_{H}(g)$. Soit $g_{*} \in \overline{\widehat{O}_{G}(g)}$, et soit $\left\{g_{n}\right\}_{n \in \mathbb{N}}$ une suite dans $G$ telle que $\lim _{n \rightarrow+\infty} g_{n}^{-1} g g_{n}=g_{*}$. Puisque le groupe $H G_{g} \backslash G$ est fini, quitte à remplacer la suite $\left\{g_{n}\right\}$ par l'une de ses sous-suites, on peut supposer que $g_{n} \in\left(H G_{g}\right) y(n \in \mathbb{N})$ pour un $y \in G$ indépendant de $n$. Quitte à remplacer, pour chaque $n \in \mathbb{N}, g_{n}$ par $g_{n}^{\prime} \in G_{g} g_{n}$, on peut supposer que $g_{n} \in H y(n \in \mathbb{N})$. Puisque $\lim _{n \rightarrow+\infty} g_{n}^{-1} g g_{n}=g_{*}$, on a $\lim _{n \rightarrow+\infty} y g_{n}^{-1} g g_{n} y^{-1}=y g_{*} y^{-1} \in \overline{\mathcal{O}_{H}(g)}$. Par conséquent, pour chaque $G$-orbite $\mathcal{O}$ contenue dans $\overline{O_{G}(g)}$, l'intersection $\mathcal{O} \cap$ $\overline{\mathrm{O}_{H}(g)}$ est non vide. On en déduit que la $H$-orbite $\mathrm{O}_{H}(g)$ est fermée dans $A$ si et seulement si la $G$-orbite $\mathfrak{O}_{G}(g)$ est fermée dans $A$; i.e., si et seulement si $g$ est $G$-fermé. (On peut montrer que $\overline{O_{H}(g)}$ contient une unique $H$-orbite fermée dans $A$, celle dont la dimension est minimale; mais nous n'en aurons pas besoin ici.) Puisque les distributions $\Theta_{\pi}(\pi \in \epsilon(H))$ sont $H$-invariantes sur $H$, il suffit de montrer qu'elles sont intégrables au voisinage des éléments $G$-fermés de $H$.

Soit $x \in H$ un élément $G$-fermé. Soient $Q_{1}(t), \ldots, Q_{r}(t)$ les composantes irréductibles du polynôme minimal réduit de $x$. Pour $i=1, \ldots, r$, on pose $V_{i}=$ 
$\operatorname{ker}\left\{Q_{i}(x): V \rightarrow V\right\}$; c'est un sous- $D$-espace vectoriel de $V$. On a la décomposition $V=\bigoplus_{i=1}^{r} V_{i}$. Soit $\mathfrak{m}$ la sous- $F$-algèbre de Lévi de $A$ définie par $\mathfrak{m}=\{g \in$ $\left.A: g\left(V_{i}\right) \subset V_{i}, i=1, \ldots, r\right\}$. On a une identification canonique $\mathfrak{m}=\bigoplus_{i=1}^{r} A_{i}$ avec $A_{i}=\operatorname{End}_{D}\left(V_{i}\right)$. Soit $B=\{g \in A: x g-g x=0\}$. On a l'inclusion $B \subset \mathfrak{m}$, laquelle entraîne la décomposition $B=\bigoplus_{i=1}^{r} B_{i}, B_{i}=B \cap A_{i}$. Chaque $B_{i}$ est une $F$-algèbre simple, et son centre $F_{i}$ est une extension finie de $F$ isomorphe à $F[t] / Q_{i}(t)$. Écrivons $x=\bigoplus_{i=1}^{r} x_{i}$ avec $x_{i} \in B_{i}$; on a $F_{i}=F\left[x_{i}\right]$.

Soit $i \in\{1, \ldots, r\}$. Fixons un caractère additif $\Psi_{F_{i}}$ de conducteur $\mathfrak{p}_{F_{i}}$ et posons $\Psi_{B_{i}}=\Psi_{F_{i}} \circ \operatorname{tr}_{B_{i} / F_{i}}^{\prime}$. Posons $\Psi_{A_{i}}=\Psi_{F}$ otr $_{A_{i} / F}^{\prime}$, et soit $s_{i}: A_{i} \rightarrow B_{i}$ l'homomorphisme $^{\prime}$ hom surjectif de $\left(B_{i}, B_{i}\right)$-bimodules défini par $\Psi_{B_{i}}\left(s_{i}(y) b\right)=\Psi_{A_{i}}(y b)$ pour tout $(y, b) \in$ $A_{i} \times B_{i}$. L'application $\tilde{s}=s_{1} \times \cdots \times s_{r}: \mathfrak{m} \rightarrow B$ est un homomorphisme surjectif de $(B, B)$-bimodules, de noyau ad $x(\mathfrak{m})$. Pour $i=1, \ldots, r$, choisissons un élément $\varrho_{i} \in A_{i}^{\times}$tel que $s_{i}\left(\varrho_{i}\right)=1$, et posons $\varrho=\bigoplus_{i=1}^{r} \varrho_{i} \in \mathfrak{m}^{\times}$. Comme en [Lemaire 2004, 7], on montre que pour tout voisinage ouvert $\mathfrak{U}$ de 0 dans $B$ suffisamment petit, on a l'inclusion $(1+\mathfrak{U} \varrho) \subset H$ et l'application $\zeta: H \times \mathfrak{U} \rightarrow H,(g, u) \mapsto$ $g^{-1}(1+u \varrho) x g$ est partout submersive. La suite est une simple adaptation de la construction précédente (ibidem). Notons qu'en général, le groupe $H_{x}$ n'est pas de la forme $\prod_{i=1}^{r} H_{i}$ pour des sous-groupes ouverts distingués $H_{i} \subset B_{i}^{\times}$(en revanche, il existe toujours un tel sous-groupe $\prod_{i=1}^{r} H_{i} \subset B^{\times}$tel que $\prod_{i=1}^{r} H_{i} \subset H_{x}$ ); mais ceci ne change rien à la construction. En définitive, on obtient :

Thórème 3.10 [Lemaire 2004, 7.1]. Soit $\pi \in \epsilon(H)$. La distribution $\Theta_{\pi}$ est localement intégrable sur $H$ (avec décomposition au voisinage des éléments $G$-fermés de $H$ comme en 3.9), localement constante sur $H_{\mathrm{r}}$.

Une version du théorème 3.10 pour les distributions $H$-invariantes sur $A$. Il s'agit d'adapter la démonstration du théorème 3.10, de manière à pouvoir achever la démonstration de la proposition 2.1.

Soit $x \in A$ un élément pur; notons que l'on peut avoir $x=0$. Reprenons la construction (et les notations) du début de la section 3, en les modifiant de la manière suivante. Pour $u \in B$, on note $\Phi_{u}: W \times B \rightarrow A$ l'application $F$-linéaire définie par

$$
\Phi_{u}(v, b)=(x+u \varrho) v-v(x+u \varrho)+b \varrho .
$$

Puisque $A=\operatorname{ad} x(A) \oplus B \varrho$, l'application $\Phi_{0}$ est bijective. Soit $\mathfrak{U}$ le voisinage ouvert de 0 dans $B$ formé des $u \in B$ tels que $\operatorname{det}_{F}\left(\Phi_{u} \circ \Phi_{0}^{-1} ; A\right) \neq 0$. On pose $\mathfrak{U}_{b}=b^{-1} \mathfrak{U} b\left(b \in H_{x}\right)$. Pour $b \in H_{x}$, l'application $\zeta_{b}: H \times \mathfrak{U}_{b} \rightarrow A,(h, u) \mapsto$ $h^{-1}\left(x+u b^{-1} \varrho b\right) h$ est partout submersive (calcul facile ; cf. [Lemaire 2004, 2.2 et 2.3]). Soit $\mathfrak{O} \subset \mathfrak{U}$ un voisinage ouvert compact de 0 dans $B$. Pour $T \in J_{H}(A)$ et $k \in \mathbb{Z}$ tel que $\varpi^{\prime k} \Xi_{\natural} \subset H_{x} \mathfrak{O}$, la famille de submersion $\left\{\zeta_{b}\right\}_{b \in H_{x}}$ permet de définir, comme en [Lemaire 2004, 2], une distribution $T_{\natural, k}$ sur $B$ à support dans $\varpi^{\prime k} \Xi_{\sharp}$. 
Soit $\mathscr{K}$ un sous-groupe ouvert compact de $(A,+)$. La formule de Plancherel pour $\mathscr{K}$ implique que

$$
\operatorname{vol}\left(\mathscr{K}, d_{A} g\right) T=\sum_{\chi \in \epsilon(\mathscr{K})}(T * \chi) d_{A} g \quad(T \in \mathscr{D}(A))
$$

avec $T * \chi(y)=\int_{A} \chi(y-g) d T^{\prime}(g)(y \in A)$. Et la proposition 3.1 reste vraie, mutatis mutandis. On en déduit la version suivante du corollaire 3.2 :

Proposition 3.11. Soient $T \in J_{H}(A)$ et $k \in \mathbb{Z}$ tel que $\varpi^{\prime k} \Xi_{\natural} \subset{ }^{H_{x}} \mathfrak{O}$. Soient $\mathfrak{B}$ un sous-groupe ouvert compact de B tel que $\mathfrak{B} \subset \varpi^{\prime k} \Xi_{\natural} \cap \mathfrak{O}$ et $\mathfrak{B} \varrho \subset \mathcal{K}$, et $\chi_{\natural} \in \epsilon(\mathfrak{B})$ un caractère tel que $\left\langle\chi_{\natural}, T_{\natural, k}\right\rangle \neq 0$. Alors il existe un caractère $\chi \in \epsilon(\mathscr{K})$ tel que

$$
T * \chi(x) \neq 0 \quad \text { et } \int_{\mathfrak{B}} \chi_{\natural}(u) \chi(u \varrho) d_{B} u \neq 0 .
$$

Démonstration. Voir la démonstration de [Lemaire 2004, 2.8]. Notons que puisque $T * \chi(x+k)=\chi(k) T * \chi(x)(k \in \mathscr{K})$, la condition $\left.T * \chi\right|_{x+\mathscr{K}} \neq 0$ obtenue en suivant cette démonstration-là est équivalente à $T * \chi(x) \neq 0$.

Tout caractère $\chi \in \epsilon(\mathscr{K})$ est de la forme $k \mapsto \Psi_{A}(z k)$ pour un $z \in A$ déterminé de manière unique modulo $\mathscr{K}^{\vee}=\left\{y \in A: \Psi_{A}(y \mathscr{K})=1\right\}$; on pose alors $Z_{\chi}=z+\mathscr{K}^{\vee} \in$ $A / \mathscr{K}^{\vee}$. On a donc $\chi^{\vee}=\operatorname{vol}\left(\mathscr{K}, d_{A} g\right) \mathbf{1}_{Z_{\chi}}(\chi \in \epsilon(\mathscr{K}))$. Pour $a \in \mathbb{Z}$ et $\chi \in \epsilon\left(\mathfrak{A}^{a}\right)$, on note $n(\chi)$ le plus petit entier $b \geq a$ tel que $\left.\chi\right|_{\mathfrak{A}^{b}}=1$. Et pour $a, b \in \mathbb{Z}$ tels que $b \geq a$, on pose $\epsilon_{b}\left(\mathfrak{A}^{a}\right)=\left\{\chi \in \epsilon\left(\mathfrak{A}^{a}\right): n(\chi)=b\right\} ;$ pour $\chi \in \epsilon\left(\mathfrak{A}^{a}\right)$, on a $\chi \in \epsilon_{b}\left(\mathfrak{A}^{a}\right)$ si et seulement si $Z_{\chi} \subset \mathfrak{A}^{(1 / d)-b} \backslash \mathfrak{A}^{(1 / d)-b+1}$.

Proposition 3.12. Il existe un entier $d_{0}=d_{0}(x, \mathfrak{A})$ tel que pour tous $a \in \mathbb{Z}, b \in \mathbb{Z}_{>a}$, $T \in J_{H}(A)$ et $\chi \in \epsilon_{b}\left(\mathfrak{A}^{a}\right)$ tels que $K^{b-a} \subset H$ et $T * \chi(x) \neq 0$, on a $Z_{\chi} \subset B+$ $\mathfrak{A}^{a-b+d_{0}}$.

Démonstration. Pour $T \in \mathscr{D}(A)$ et $a \in \mathbb{Z}$, notons $T_{x, a} \in \mathscr{D}\left(\mathfrak{A}^{a}\right)$ la distribution définie par $d T_{x, a}(y)=d T(x+y)\left(y \in \mathfrak{A}^{a}\right)$.

Soit $T \in J_{H}(A)$ et $a \in \mathbb{Z}$. Pour $r \in \frac{1}{d} \mathbb{Z} \cap \mathbb{R}_{>a}$ tel que $K^{r-a} \subset H, t \in \mathfrak{A}^{a}$ et $y \in \mathfrak{A}^{r-a}$, on a

$$
\begin{aligned}
\left\langle\mathbf{1}_{t+\mathfrak{A}^{r}}, T_{x, a}\right\rangle & =\left\langle\mathbf{1}_{x+t+\mathfrak{A}^{r}}, T\right\rangle=\left\langle\mathbf{1}_{(1+y)^{-1}\left(x+t+\mathfrak{A}^{r}\right)(1+y)}, T\right\rangle \\
& =\left\langle\mathbf{1}_{x+t+x y-y x+\mathfrak{A}^{r}}, T\right\rangle=\left\langle\mathbf{1}_{\left.t+x y-y x+\mathfrak{A}^{r}, T_{x, a}\right\rangle .}\right.
\end{aligned}
$$

On en déduit comme dans la démonstration de [Lemaire 2004, 3.2], que

$$
\operatorname{Supp}\left(T_{x, a}^{\vee}\right) \cap \mathfrak{A}^{(1 / d)-r} \subset\left\{g \in A: x g-g x \in \mathfrak{A}^{(1 / d)-r+a}\right\} .
$$

Puisque $\operatorname{ker}\{\operatorname{ad} x: A \rightarrow A\}=B$, il existe un entier $c$ tel que pour tout $g \in A$, on a l'implication $x g-g x \in \mathfrak{A}^{1 / d} \Rightarrow g \in B+\mathfrak{A}^{c}$. D'où l'inclusion

$$
\operatorname{Supp}\left(T_{x, a}^{\vee}\right) \cap \mathfrak{A}^{(1 / d)-b} \subset B+\mathfrak{A}^{c-b+a} \quad\left(b \in \mathbb{Z}_{>a} \text { tel que } K^{b-a} \subset H\right) .
$$


Soient maintenant $b \in \mathbb{Z}_{>a}$ et $\chi \in \epsilon_{b}\left(\mathfrak{A}^{a}\right)$ tels que $K^{b-a} \subset H$ et $T * \chi(x) \neq 0$. On a $T * \chi(x)=\left\langle\bar{\chi}, T_{x, a}\right\rangle=\left\langle\chi^{\vee}, T_{x, a}^{\vee}\right\rangle$, donc $\operatorname{Supp}\left(T_{x, a}^{\vee}\right) \cap Z_{\chi} \neq \varnothing$. Puisque $Z_{\chi} \subset \mathfrak{A}^{(1 / d)-b}$, on obtient que $Z_{\chi} \cap\left(B+\mathfrak{A}^{c-b+a}\right) \neq \varnothing$. Posons $d_{0}=\inf \left\{c, \frac{1}{d}-a\right\}$. Comme $Z_{\chi}+\mathfrak{A}^{(1 / d)-a}=Z_{\chi}$, on a l'inclusion $Z_{\chi} \subset B+\mathfrak{A}^{d_{0}-b+a}$.

Une distribution $T \in \mathscr{D}(A)$ est dite $\mathscr{K}$-admissible en $x$ si elle vérifie la propriété suivante : il existe un entier $m_{0}=m_{0}(T, \mathscr{K}, x)$ tel que pour tous $a \in \mathbb{Z}_{\geq m_{0}}$ et $\chi \in \epsilon\left(\varpi^{a \mathscr{K}}\right)$ tels que $T * \chi(x) \neq 0$, on a $Z_{\chi} \cap \mathfrak{N} \neq \varnothing$.

Continuons avec les notations introduites dans le paragraphe "Étude locales des réductions à $B$ des caractères de $H$ " (page 99). Pour $T \in J_{H}(A)$, notons $\theta_{T}$ la distribution $H_{x}$-invariante sur $B$ définie par $\theta_{T}=T_{\natural, k_{0}}$.

Proposition 3.13. Soit $\mathscr{V}$ un voisinage ouvert fermé de $\mathfrak{N}_{\natural} \backslash\{0\}$ dans $B \backslash\{0\}$ tel que $F^{\times \mathcal{V}}=\mathscr{V}$, et soit $T \in J_{H}(A)$ une distribution $\mathfrak{A}$-admissible en $x$. Il existe un entier a tel que pour tout $\chi_{\natural} \in \epsilon\left(\mathfrak{A}_{\natural}^{a+a_{0}}\right)$ tel que $\left\langle\bar{\chi}_{\natural}, \theta_{T}\right\rangle$, on a l'inclusion $\operatorname{Supp}\left(\chi_{\natural}^{\vee}\right) \subset$ $\mathscr{V} \cup \mathfrak{A}_{\natural}^{\mu}$ avec $\mu=\delta^{\prime-1}-2 a-a_{0}+1$.

Démonstration. Posons $\mathscr{V}^{*}=\mathscr{V} \cap\left(\mathfrak{A} \backslash \mathfrak{A}^{1}\right)$. Soient $d_{0}=d_{0}(x, \mathfrak{A})$ et $v=v\left(s, \mathscr{V}^{*}\right)$ des entiers comme dans la proposition 3.12 et le lemme 3.6. Soit un entier $a>0$ tel que :

(1) $\mathfrak{A}_{\natural}^{a+a_{0}} \subset \omega^{\prime k_{0}} \Xi_{\natural} \cap \mathfrak{O}$,

(2) $a \geq \frac{1}{d} v-d_{0}+\left[\frac{1}{d}\right]$ où [] désigne la partie entière,

(3) $\mathscr{V}^{*}+\mathfrak{A A}_{\natural}^{\delta^{\prime-1}+a-a_{0}}=\mathfrak{V}^{*}$,

(4) $K^{a} \subset H$ et $a \geq m_{0}(T, \mathfrak{A}, x)$.

D'après la démonstration de [Lemaire 2004, 4.2], cet entier $a$ convient.

Proposition 3.14 [Lemaire 2004, 4.3]. Soit $T \in J_{H}(A)$ une distribution $\mathfrak{A}$-admissible en $x$. La distribution $\theta_{T}$ est intégrable au voisinage de 0 dans $B$. Plus précisément, il existe un entier $m$ et des constantes $c_{n}(T)\left(n \in \mathcal{N}_{H, \downarrow}\right)$ tels que

$$
\mathbf{1}_{\mathfrak{A}_{\natural}^{m}} \cdot\left(\theta_{T}-\sum_{n \in \mathcal{N}_{H, \sharp}} c_{n}(T) \Theta_{\natural, n}^{\vee}\right)=0 .
$$

Corollaire 3.15 [Lemaire 2004, 4.4]. La distribution T est intégrable au voisinage de $x$ dans $H$. Plus précisément, il existe un entier $m$ tel que

$$
T(x+u \varrho)=\sum_{n \in \mathcal{N}_{H, \natural}} c_{n}(T) \Theta_{\natural, n}^{\vee}(u)
$$

pour presque tout $u \in \mathfrak{A}_{\natural}^{m}$ (resp. pour tout $u \in \mathfrak{A}_{\natural}^{m} \cap B^{\prime}$ ). 
Le paragraphe "Réduction aux éléments $G$-purs de $H$ (descente parabolique)" (page 101) s'étend sans aucune difficulté à la situation présente. Notons que si $x$ est élément fermé de $A$, alors $x$ se décompose en $\bigoplus_{i=1}^{r} x_{i}$ comme plus haut. Mais on peut avoir $x_{j}=0$ pour un (unique) indice $j$; c'est pourquoi, pour $x$ pur, on a remplacé $\left(1+u b^{-1} \varrho b\right) x$ par $x+b^{-1} u b \varrho$ dans la définition de $\zeta_{b}$. En définitive, on obtient :

Thórème 3.16. Soit $T \in J_{H}(A)$ une distribution $\mathfrak{A}$-admissible en $x$ pour tout élément fermé $x \in A$. Alors $T$ est localement intégrable sur A (avec décomposition au voisinage des éléments fermés de A comme au corollaire 3.15), localement constante sur $A_{\mathrm{r}}$.

Démonstration de la proposition 2.1. D'après 2.7 et 2.33, il nous reste à montrer que pour tous $T \in J_{H}^{*}(A)$ et $y \in A \backslash\{0\}$, la distribution $T^{\vee}$ est intégrable au voisinage de $y$ dans $A$.

Soit $\mathscr{K}$ un sous-groupe ouvert compact de $(A,+)$.

Lemme 3.17. Soient $b \in \mathbb{Z}$ tel que $\mathscr{K} \subset \mathfrak{A}^{(1 / d)-b}, T \in J_{H}\left(\varpi^{b+1} \Xi\right)$ et $\chi \in \epsilon(\mathscr{K})$. Si $T^{\vee} * \chi \neq 0$, alors $Z_{\chi} \cap \mathfrak{N} \neq \varnothing$.

Démonstration. On a (calcul facile)

$$
T^{\vee} \chi \chi(y)=\operatorname{vol}\left(\mathcal{K}, d_{A} g\right) \int_{Z_{\chi}} \overline{\Psi_{A}(g y)} d T(g) \quad(y \in A) .
$$

Par conséquent, si $T^{\vee} * \chi \neq 0$, alors $Z_{\chi} \cap \varpi^{b+1} \Xi \neq \varnothing$. D'après [Lemaire 2004, 1.2 et démonstration de 1.3], on a $\varpi^{b+1} \Xi \subset G\left(\mathfrak{A}^{b}\right) \subset \mathfrak{N}+\mathfrak{A}^{b}$. D'où le lemme puisque $\mathfrak{A}^{b} \subset \mathscr{K}^{\vee}$ et $Z_{\chi}+\mathscr{K}^{\vee}=Z_{\chi}$.

Soit maintenant $T \in J_{H}^{*}(A)$. Il existe un entier $b$ tel que $\operatorname{Supp}(T) \subset \varpi^{b+1} \Xi$. Soit $m_{0}=1-b$. Alors pour tous $a \in \mathbb{Z}_{\geq m_{0}}$ et $\chi \in \epsilon\left(\mathfrak{A}^{a}\right)$ tels que $T^{\vee} * \chi \neq 0$, on a $Z_{\chi} \cap \mathfrak{N} \neq \varnothing$. En particulier, la distribution $T^{\vee}$ est $\mathfrak{A}$-admissible en tout point de $A$. On peut donc appliquer le théorème 3.16. Ce qui achève la démonstration de la proposition 2.1.

\section{Intégrabilité locale des caractères de $G^{\prime}$ : une méthode générale}

Cette section est indépendante de celles qui la précèdent. En particulier, certaines notations introduites ci-dessous, bien que déjà utilisées dans les sections 1 à 3 , désignent ici des objets beaucoup plus généraux que ceux qu'elles y désignaient.

Soit $H$ un groupe topologique localement profini possédant une base dénombrable d'ouverts, et soient $G^{\prime}, C \subset H$ deux sous-groupes fermés distingués tels que :

- $G^{\prime} \cap C=\{1\}$,

- le groupe $C$ est central dans $H$ et discret (pour la topologie induite), 
- le groupe $C G^{\prime} \backslash H$ est commutatif et compact (pour la topologie quotient). On pose $\tilde{G}=C G^{\prime}$ (produit direct). Soit $\left\{K^{a}: a \in \mathbb{Z}_{\geq 0}\right\}$ une famille de sous-groupes ouverts compacts de $H$ telle que :

- pour $a \geq 1, K^{a}$ est distingué dans $K^{0}$,

- pour tout voisinage ouvert $\Omega$ de 1 dans $H$, il existe un entier $a \geq 0$ tel que $K^{a} \subset \Omega$.

Puisque $H$ possède une base dénombrable d'ouverts, une telle famille existe. On pose $K=K^{0}$ et $K^{\prime a}=K^{a} \cap G^{\prime}\left(a \in \mathbb{Z}_{\geq 0}\right)$. Puisque $C$ est discret dans $H$, il existe un entier $a_{0} \geq 0$ tel que $K^{a_{0}} \cap C=\{1\}$. On suppose que les groupes $H$ et $G^{\prime}$ sont unimodulaires. Le groupe $\tilde{G}$ est alors lui aussi unimodulaire.

Soit $\left(\pi^{\prime}, W\right)$ une représentation complexe lisse admissible irréductible de $G^{\prime}$. Notons $(\tilde{\pi}, W)$ la représentation de $\tilde{G}$ définie par $\tilde{\pi}(z g)=\pi^{\prime}\left(g^{\prime}\right)$ pour tous $z \in C$ et $g^{\prime} \in G^{\prime}$. On suppose que la représentation $(\tilde{\pi}, W)$ de $\tilde{G} s^{\prime}$ étend en une représentation lisse $(\pi, W)$ de $H$. Puisque $\pi^{\prime}$ est admissible, $\pi$ l'est aussi. Soit $\operatorname{ind}_{\tilde{G}}^{H} W$ le $\mathbb{C}$-espace vectoriel formé des fonctions $\phi: H \rightarrow W$ telles que :

- $\phi(\tilde{g} h)=\tilde{\pi}(\tilde{g})(\phi(h))$ pour tous $\tilde{g} \in \tilde{G}, h \in H$,

- il existe un sous-groupe ouvert compact $K_{\phi} \subset H$ tel que $\phi(h k)=\phi(h)$ pour tous $h \in H, k \in K_{\phi}$.

Soit $(\sigma, V)=\left(\operatorname{ind}_{\tilde{G}}^{H} \tilde{\pi}, \operatorname{ind}_{\tilde{G}}^{H} W\right)$ la représentation de $H$ définie par $\sigma(h)(\phi)\left(h^{\prime}\right)=$ $\phi\left(h^{\prime} h\right)\left(h, h^{\prime} \in H ; \phi \in V\right)$; i.e., l'induite lisse de $\tilde{\pi}$ à $H$. Puisque $\tilde{G}$ est cocompact dans $H$, la représentation $\sigma$ est admissible. Posons $\epsilon=\epsilon(\tilde{G} \backslash H)$.

Lemme 4.1. Les représentations $\pi \otimes \chi(\chi \in \epsilon)$ sont deux à deux non équivalentes, et l'on a $\sigma \simeq \bigoplus_{\chi \in \epsilon} \pi \otimes \chi$.

Démonstration. Soient $\chi, \chi^{\prime} \in \epsilon$, et $\gamma \in \operatorname{Hom}_{H}\left(\pi \otimes \chi, \pi \otimes \chi^{\prime}\right)$ tel que $\gamma \neq 0$. Par restriction, $\gamma$ définit un élément de $\operatorname{Hom}_{\tilde{G}}(\tilde{\pi}, \tilde{\pi})$, par suite $\gamma \in \mathbb{C}^{\times}$(lemme de Schur) et $\chi=\chi^{\prime}$. Les représentations $\pi \otimes \chi(\chi \in \epsilon)$ sont donc deux à deux non équivalentes.

Posons $\mathfrak{F}=C^{\infty}(\tilde{G} \backslash H)$. L'application

$$
\mathfrak{F} \otimes_{\mathbb{C}} W \stackrel{\iota}{\longrightarrow} V, \quad f \otimes w \mapsto(h \mapsto f(h) \pi(h)(w))
$$

est $\mathbb{C}$-linéaire, et $H$-équivariante pour l'opération de $H$ sur $\mathfrak{F} \otimes_{\mathbb{C}} W$ donnée par

$$
h \cdot(f \otimes w)=f_{h} \otimes \pi(h)(w), \quad f_{h}\left(h^{\prime}\right)=f\left(h^{\prime} h\right) \quad\left(h, h^{\prime} \in H\right) .
$$

L'injectivité de $\iota$ est claire. Montrons la surjectivité. Soit $X \subset H$ une partie compacte telle que $H=\tilde{G} X$. Soit $\phi \in V$. L'ensemble $Y=\left\{\pi\left(x^{-1}\right)(\phi(x)): x \in X\right\}$ est fini, par conséquent il existe un sous-groupe ouvert compact $J \subset H$ tel que $\phi(h j)=\phi(h)(h \in H, j \in J)$ et $\pi(j)(y)=y(y \in Y, j \in J)$. Soient $x_{1}, \ldots, x_{r} \in X$ un système de représentants des doubles classes $\tilde{G} \backslash H / J$. Écrivons $\phi=\sum_{i=1}^{r} \phi_{i}$ 
$\operatorname{avec} \operatorname{Supp}\left(\phi_{i}\right) \subset \tilde{G} x_{i} J$. Pour $i=1, \ldots, r$, on a $\phi_{i}=\iota\left(\mathbf{1}_{\tilde{G} \backslash \tilde{G} x_{i} J} \otimes w_{i}\right)$ avec $w_{i}=$ $\pi\left(x_{i}^{-1}\right)\left(\phi_{i}\left(x_{i}\right)\right)$. D'où la surjectivité de $\iota$.

Les éléments de $\epsilon$ forment une $\mathbb{C}$-base de $\mathfrak{F}$, et pour $\chi \in \epsilon$, on a $h \cdot(\chi \otimes w)=$ $\chi \otimes(\pi \otimes \chi)(h)(w)(w \in W)$. D'où lemme.

Pour $a \in \mathbb{Z}_{\geq a_{0}}$ et $\chi \in \epsilon$, on pose $\chi_{a}=\left.\chi\right|_{K^{a}} \in \epsilon\left(K^{\prime a} \backslash K^{a}\right) \subset \epsilon\left(K^{a}\right)$; et l'on note $V^{K^{a}} \subset V$ le sous-espace des points fixés par $K^{a}$ et $W\left(\chi_{a}^{-1}\right) \subset W$ la composante $\chi_{a}^{-1}$-isotypique de $\pi$. D'après 13 lemme 4.1 , on a la décomposition

$$
V^{K^{a}} \simeq \bigoplus_{\chi \in \epsilon} W\left(\chi_{a}^{-1}\right) \quad\left(a \in \mathbb{Z}_{\geq a_{0}}\right)
$$

Puisque la représentation $\sigma$ est admissible, l'ensemble des $\chi$ intervenant dans la décomposition (4.2) est fini ; i.e., $W\left(\chi_{a}^{-1}\right)=0$ pour presque tout $\chi \in \epsilon$. Pour $a \in \mathbb{Z}_{\geq a_{0}}$, on pose $\epsilon_{a}=\epsilon\left(\tilde{G} K^{a} \backslash H\right) \subset \epsilon$, et l'on note $b(\pi, a)$ le plut petit entier $b \geq a$ tel que $\left\{\chi \in \epsilon: W\left(\chi_{a}^{-1}\right) \neq 0\right\} \subset \epsilon_{b}$. On a donc les inclusions

$$
W^{K^{a}} \subset W^{K^{\prime a}} \subset W^{K^{b(\pi, a)}} \quad\left(a \in \mathbb{Z}_{\geq a_{0}}\right) ;
$$

précisément, $b(\pi, a)$ est le plus petit entier $b \geq a$ tel que $W^{K^{\prime a}} \subset W^{K^{b}}$.

Soient $d h$ et $d \tilde{g}$ les mesures de Haar respectivement sur $H$ et $\tilde{G}$ normalisées par $K$. Posons $d k=\left.d h\right|_{K}, d g^{\prime}=\left.d \tilde{g}\right|_{G^{\prime}}$ et $d k^{\prime}=\left.d g^{\prime}\right|_{K^{\prime}}$. Soit $\kappa=(H: \tilde{G} K)$. Pour $a \in \mathbb{Z}_{\geq a_{0}}$, on a

$$
\left|\epsilon_{a}\right|=\left(H: \tilde{G} K^{a}\right)=\kappa\left(\tilde{G} K: \tilde{G} K^{a}\right)=\kappa \frac{\left(K: K^{a}\right)}{\left(\tilde{K}: K^{\prime a}\right)}=\kappa c_{a}^{-1} c_{a}^{\prime}
$$

$\operatorname{avec} \tilde{K}=K \cap \tilde{G}, c_{a}=\operatorname{vol}\left(K^{a}, d k\right)$ et $c_{a}^{\prime}=\operatorname{vol}\left(K^{\prime a}, d k^{\prime}\right)$.

Pour toute représentation complexe lisse admissible $\tau$ de $H$, on note $\Theta_{\tau} \in \mathscr{D}(H)$ la distribution trace $(\tau d h)$. De même, pour toute représentation complexe lisse admissible $\tilde{\tau}$ de $\tilde{G}\left(\operatorname{resp} . \tau^{\prime}\right.$ de $\left.G^{\prime}\right)$, on pose $\Theta_{\tilde{\tau}}=\operatorname{trace}(\tilde{\tau} d \tilde{g}) \in \mathscr{D}(\tilde{G})$ et $\Theta_{\tau^{\prime}}=$ trace $\left(\tau^{\prime} d g^{\prime}\right) \in \mathscr{D}\left(G^{\prime}\right)$. Pour simplifier l'écriture, si $\tau$ est une représentation lisse de $H$ et $f \in C_{\mathrm{c}}^{\infty}(H)$, on écrira $\tau(f)$ au lieu $\tau(f d h)\left(=\int_{H} f(h) \tau(h) d h\right)$.

Pour $f \in C_{\mathrm{c}}^{\infty}(H)$ et $\Omega \subset H$ une partie ouverte compacte, on note $f_{\Omega} \in C_{\mathrm{c}}^{\infty}(H)$ la fonction définie par $f_{\Omega}(y)=\operatorname{vol}(\Omega, d h)^{-1} \int_{\Omega} f\left(h^{-1} y h\right) d h$. Et pour $f \in C_{\mathrm{c}}^{\infty}(\tilde{G})$, on note ${ }_{C} f \in C_{\mathrm{c}}^{\infty}\left(G^{\prime}\right)$ la fonction définie par ${ }_{C} f\left(g^{\prime}\right)=\sum_{z \in C} f\left(z g^{\prime}\right)$ (puisque $C$ est discret et $\operatorname{Supp}(f)$ est compact, cette somme est finie).

Supposons que $h_{1}, \ldots, h_{s} \in H$ forment un système de représentants des doubles classes $\tilde{G} \backslash H / K^{a_{0}}$. Puisque $\tilde{G}$ et $\tilde{G} K^{a_{0}}$ sont distingués dans $H$, on a

$$
H=\bigsqcup_{i=1}^{s} h_{i} \tilde{G} K^{a_{0}}=\bigsqcup_{i=1}^{s} \tilde{G} K^{a_{0}} h_{i} .
$$

Soit $X=\bigsqcup_{i=1}^{s} K^{a_{0}} h_{i}$. 
Proposition 4.4. Soit $\left(\pi_{1}^{\prime}, W_{1}\right)$ une représentation complexe lisse admissible de $G^{\prime}$. Soit $\tilde{\pi}_{1}$ la représentation de $\tilde{G}$ définie par $\tilde{\pi}_{1}\left(z g^{\prime}\right)=\pi_{1}^{\prime}\left(g^{\prime}\right)$ pour tous $z \in C$ et $g^{\prime} \in G^{\prime}$, et soit $\sigma_{1}=\operatorname{ind}_{\tilde{G}}^{H} \tilde{\pi}_{1}$. Pour $f \in C_{\mathrm{c}}^{\infty}(H)$, on a

$$
\left\langle f, \Theta_{\sigma_{1}}\right\rangle=\kappa\left\langle\left. f_{X}\right|_{\tilde{G}}, \Theta_{\tilde{\pi}_{1}}\right\rangle=\kappa\left\langle C\left(\left.f_{X}\right|_{\tilde{G}}\right), \Theta_{\pi_{1}^{\prime}}\right\rangle .
$$

Démonstration. Soit $C^{\infty}\left(X, W_{1}\right)$ le $\mathbb{C}$-espace vectoriel formé des fonctions localement constantes $X \rightarrow W_{1}$, canoniquement identifié à $C^{\infty}(X) \otimes \mathbb{C} W_{1}$. Pour tout sousgroupe ouvert $J \subset K^{a_{0}}$, on pose $C\left(J \backslash X, W_{1}\right)=C(J \backslash X) \otimes_{\mathbb{C}} W_{1} \subset C^{\infty}\left(X, W_{1}\right)$. L'application $V_{1}=\operatorname{ind}_{\tilde{G}}^{H} W_{1} \rightarrow C^{\infty}\left(X, W_{1}\right),\left.\phi \mapsto \phi\right|_{X}$ est un $\mathbb{C}$-isomorphisme de $V_{1}$ sur le sous-espace vectoriel $\mathfrak{H} \subset C^{\infty}\left(X, W_{1}\right)$ formé des fonctions $\eta: X \rightarrow W_{1}$ telles que $\eta\left(k^{\prime} x\right)=\pi_{1}^{\prime}\left(k^{\prime}\right)(\eta(x))$ pour tous $k^{\prime} \in K^{\prime a_{0}}$ et $x \in X$. Soit $\rho: K^{a_{0}} \rightarrow$ $\operatorname{Aut}_{\mathbb{C}}\left(C^{\infty}(X)\right)$ la représentation (admissible) définie par $\rho(k)(\alpha)(x)=\alpha\left(k^{-1} x\right)$ $\left(k \in K^{a_{0}}, \alpha \in C^{\infty}(X), x \in X\right)$. Le $\mathbb{C}$-endomorphisme $u$ de $C^{\infty}\left(X, W_{1}\right)$ défini par $u(\alpha \otimes w)=c_{0}^{\prime-1} \int_{K^{\prime a_{0}}} \rho\left(k^{\prime}\right)(\alpha) \otimes \pi_{1}^{\prime}\left(k^{\prime}\right)(w) d k^{\prime}\left(\alpha \in C^{\infty}(X), w \in W_{1}\right)$ avec $c_{0}^{\prime}=c_{a_{0}}^{\prime}$, est un projecteur de $C^{\infty}\left(X, W_{1}\right)$ sur $\mathfrak{H}:$ on a $\operatorname{Im} u=\mathfrak{H}$ et $\left.u\right|_{\mathfrak{H}}=\operatorname{Id}_{\mathfrak{H}}$.

Soit $f \in C_{\mathrm{c}}^{\infty}(H)$. Choisissons des entiers $a, b \geq a_{0}$ tels que $f \in C_{\mathrm{c}}\left(K^{a} \backslash H / K^{a}\right)$ et $K^{b} \subset h_{i} K^{a} h_{i}^{-1} \subset K^{a_{0}}(i=1, \ldots, s)$. On a donc $\sigma(f)\left(V_{1}\right) \subset V_{1}^{K^{a}}$ et $X K^{a}=X$. Et puisque $K^{a_{0}}$ normalise $K^{b}$, pour $\phi \in V_{1}^{K^{a}}$ on a $\phi(k x)=\phi(x)\left(k \in K^{b}, x \in X\right)$, par conséquent $\left.\phi\right|_{X} \in C\left(K^{b} \backslash X, W_{1}\right) \cap \mathfrak{H} \subset C\left(K^{b} \backslash X, W_{1}^{K^{\prime b}}\right)$. Identifions $V_{1}$ à $\mathfrak{H}$ via l'isomorphisme $\left.\phi \mapsto \phi\right|_{X}$. Puisque $C\left(K^{b} \backslash X, W_{1}^{K^{\prime b}}\right)$ est un $\mathbb{C}$-espace vectoriel de dimension finie, l'opérateur $\sigma_{1}(f) \circ u$ sur $C^{\infty}\left(X, W_{1}\right)$ est de rang fini, et l'on a l'égalité $\left\langle f, \Theta_{\sigma_{1}}\right\rangle=\operatorname{trace}\left(\sigma_{1}(f) \circ u\right)$.

Soit $W_{1}\left(K^{\prime b}\right)$ le sous-espace vectoriel de $W_{1}$ engendré par les $w-\pi_{1}^{\prime}\left(k^{\prime}\right) w$ pour $w \in W_{1}$ et $k^{\prime} \in K^{\prime b}$. On a la décomposition $W_{1}=W_{1}^{K^{\prime b}} \oplus W_{1}\left(K^{\prime b}\right)$. De même, on a la décomposition $C^{\infty}(X)=C^{\infty}(X)^{K^{b}} \oplus C^{\infty}(X)\left(K^{b}\right)$ avec $C^{\infty}(X)^{K^{b}}=C\left(K^{b} \backslash X\right)$ et $C^{\infty}(X)\left(K^{b}\right)=\left\langle\alpha-\rho(k)(\alpha): \alpha \in C^{\infty}(X), k \in K^{b}\right\rangle$. Puisque $K^{\prime b} \subset K^{b}$, on a (calcul facile) $u\left(C^{\infty}\left(K^{b} \backslash X\right) \otimes_{\mathbb{C}} W_{1}\left(K^{\prime b}\right)\right)=0$. Par ailleurs, le sous-espace vectoriel $V_{1}\left(K^{a}\right)=\left\langle\phi-\sigma_{1}(k)(\phi): \phi \in V_{1}, k \in K^{a}\right\rangle \subset V_{1}$ s'identifie au sous-espace vectoriel de $\mathfrak{H}$ formé des fonctions $\alpha: X \rightarrow W_{1}$ telles que $\int_{K^{a}} \alpha(x k) d k=0$. Puisque $K^{b}$ normalise $K^{\prime a_{0}}$ et $K^{b} x \subset x K^{a}(x \in X)$, on a l'inclusion $u\left(C^{\infty}(X)\left(K^{b}\right) \otimes_{\mathbb{C}} W_{1}\right) \subset$ $V_{1}\left(K^{a}\right)$. Puisque $\sigma_{1}(f)\left(V_{1}\left(K^{a}\right)\right)=0$, on obtient que $\left\langle f, \Theta_{\sigma_{1}}\right\rangle$ coïncide avec la trace de l'opérateur $\sigma_{1}(f) \circ u$ sur l'espace vectoriel $C\left(K^{b} \backslash X, W_{1}^{K^{\prime b}}\right)$.

Soit $\left(\pi_{1}^{\prime \vee}, W_{1}^{\vee}\right)$ la représentation contragrédiente de $\pi_{1}^{\prime}$. Le morphisme de dualité $W_{1} \times W_{1}^{\vee} \rightarrow \mathbb{C},\left(w, w^{\vee}\right) \mapsto\left\langle w, w^{\vee}\right\rangle$ induit par restriction une forme bilinéaire non dégénérée sur $W_{1}^{K^{\prime b}} \times\left(W_{1}^{\vee}\right)^{K^{\prime b}}$. Soit $(\phi, \varphi) \mapsto\langle\phi, \varphi\rangle$ la forme bilinéaire non dégénérée sur $C\left(K^{b} \backslash X, W_{1}^{K^{\prime b}}\right) \times C\left(K^{b} \backslash X,\left(W_{1}^{\vee}\right)^{K^{\prime b}}\right)$ définie par

$$
\langle\phi, \varphi\rangle=\sum_{i=1}^{s} \int_{K^{a_{0}}}\left\langle\phi\left(k h_{i}\right), \varphi\left(k h_{i}\right)\right\rangle d k .
$$


Soit $\mathfrak{B}$ une $\mathbb{C}$-base de $W_{1}^{K^{\prime b}}$, et soit $\mathfrak{B}^{\vee}=\left\{w^{\vee}: w \in \mathfrak{B}\right\}$ la $\mathbb{C}$-base de $\left(W_{1}^{\vee}\right)^{K^{\prime b}}$ duale de $\mathfrak{B}$. Soit $\mathfrak{C}_{0}$ une $\mathbb{C}$-base de $C\left(K^{b} \backslash K^{a_{0}}\right)$, et soit $\mathfrak{C}$ la $\mathbb{C}$-base de $C\left(K^{b} \backslash X\right)$ définie par $\mathfrak{C}=\left\{\alpha_{i}: \alpha \in \mathfrak{C}_{0}, i=1, \ldots, s\right\}$ avec $\operatorname{Supp}\left(\alpha_{i}\right) \subset K^{a_{0}} h_{i}$ et $\alpha_{i}\left(k h_{i}\right)=\alpha(k)$ $\left(k \in K^{a_{0}}\right)$. Posons $c=c_{b}$. Alors $\left\{c^{-1} \alpha \otimes w^{\vee}: \alpha \in \mathfrak{C}, w \in \mathfrak{B}\right\}$ est la base de $C\left(K^{b} \backslash X,\left(W_{1}^{\vee}\right)^{K^{\prime b}}\right)$ duale de $\{\alpha \otimes w: \alpha \in \mathfrak{C}, w \in \mathfrak{B}\}$ pour la dualité définie plus haut. On a donc

$$
\begin{aligned}
\left\langle f, \Theta_{\sigma_{1}}\right\rangle & =c^{-1} \sum_{\alpha \in \mathfrak{C}} \sum_{w \in \mathfrak{B}}\left\langle\sigma_{1}(f) \circ u(\alpha \otimes w), \alpha \otimes w^{\vee}\right\rangle \\
& =c^{-1} \sum_{\alpha \in \mathfrak{C}} \sum_{w \in \mathfrak{B}} \sum_{i=1}^{s} \int_{K^{a_{0}}} \alpha\left(k h_{i}\right)\left\langle\sigma_{1}(f) \circ u(\alpha \otimes w)\left(k h_{i}\right), w^{\vee}\right\rangle d k \\
& =c^{-1} \sum_{i=1}^{s} \sum_{\alpha \in \mathfrak{C}_{0}} \sum_{w \in \mathfrak{B}} \int_{K^{a_{0}}} \alpha(k)\left\langle\sigma_{1}(f) \circ u\left(\alpha_{i} \otimes w\right)\left(k h_{i}\right), w^{\vee}\right\rangle d k .
\end{aligned}
$$

Pour $(\phi, w) \in \mathfrak{C} \times \mathfrak{B}$ et $x \in X$, on a

$$
u(\alpha \otimes w)(x)=c_{0}^{\prime-1} \int_{K^{\prime a_{0}}} \alpha\left(k^{\prime-1} x\right) \otimes \pi_{1}^{\prime}\left(k^{\prime}\right)(w) d k^{\prime}
$$

et

$$
\begin{aligned}
\sigma_{1}(f) \circ u(\alpha \otimes w)(x) & =\int_{H} f(h) u(\alpha \otimes w)(x h) d h \\
& =\sum_{i=1}^{s} \int_{\tilde{G} K^{a_{0}}} f\left(x^{-1} h h_{i}\right) u(\alpha \otimes w)\left(h h_{i}\right) d h \\
& =c_{0}^{\prime-1} \sum_{i=1}^{s} \iint_{\tilde{G} \times K^{a_{0}}} f\left(x^{-1} \tilde{g} k h_{i}\right) \tilde{\pi}_{1}(\tilde{g})\left(u(\alpha \otimes w)\left(k h_{i}\right)\right) d \tilde{g} d k ;
\end{aligned}
$$

d'où l'on déduit que

$$
\begin{aligned}
\sigma_{1}(f) \circ u(\alpha \otimes w)(x) \\
\quad=c_{0}^{\prime-2} \sum_{i=1}^{s} \iiint_{\tilde{G} \times K^{a_{0} \times K^{\prime a_{0}}}} f\left(x^{-1} \tilde{g} k h_{i}\right) \alpha\left(k^{\prime-1} k h_{i}\right) \otimes \tilde{\pi}_{1}\left(\tilde{g} k^{\prime}\right)(w) d \tilde{g} d k d k^{\prime} \\
=c_{0}^{\prime-1} \sum_{i=1}^{s} \iint_{\tilde{G} \times K^{a_{0}}} f\left(x^{-1} \tilde{g} k h_{i}\right) \alpha\left(k h_{i}\right) \otimes \tilde{\pi}_{1}(\tilde{g})(w) d \tilde{g} d k .
\end{aligned}
$$

Soit $f_{X}^{*} \in C_{\mathrm{c}}^{\infty}(\tilde{G})$ la fonction définie par $f_{X}^{*}=\left.\operatorname{vol}(X, d h) f_{X}\right|_{\tilde{G}}$. Pour $\tilde{g} \in \tilde{G}$, on a $f_{X}^{*}(\tilde{g})=\sum_{i=1}^{s} \int_{K^{a_{0}}} f\left(h_{i}^{-1} k^{-1} \tilde{g} k h_{i}\right) d k$. Puisque $x^{-1} K^{b} x \subset K^{a}(x \in X)$ et 


$$
\begin{aligned}
& f \in C_{\mathrm{c}}\left(K^{a} \backslash H / K^{a}\right), \text { on a } f_{X}^{*} \in C_{\mathrm{c}}\left(K^{\prime b} \backslash \tilde{G} / K^{\prime b}\right) \text { et } \\
& \left\langle f, \Theta_{\sigma_{1}}\right\rangle=\left(c c_{0}^{\prime}\right)^{-1} \sum_{i=1}^{s} \sum_{\alpha \in \mathfrak{C}_{0}} \sum_{w \in \mathfrak{B}} \int_{K^{a_{0}}} \alpha\left(k_{1}\right) \cdots \\
& \quad \cdots\left(\iint_{\tilde{G} \times K^{a_{0}}} f\left(h_{i}^{-1} k_{1}^{-1} \tilde{g} k_{2} h_{i}\right) \alpha\left(k_{2}\right)\left\langle\tilde{\pi}_{1}(\tilde{g})(w), w^{\vee}\right\rangle d \tilde{g} d k_{2}\right) d k_{1} \\
& =c\left(c c_{0}^{\prime}\right)^{-1} \sum_{i=1}^{s} \sum_{w \in \mathfrak{B}} \iint_{\tilde{G} \times K^{a_{0}}} f\left(h_{i}^{-1} k^{-1} \tilde{g} k h_{i}\right)\left\langle\tilde{\pi}_{1}(\tilde{g})(w), w^{\vee}\right\rangle d \tilde{g} d k \\
& =c_{0}^{\prime-1} \sum_{w \in \mathfrak{B}} \int_{\tilde{G}} f_{X}^{*}(\tilde{g})\left\langle\tilde{\pi}_{1}(\tilde{g})(w), w^{\vee}\right\rangle d \tilde{g} .
\end{aligned}
$$

Or $f_{X}^{*}=\left.s c_{0} f_{X}\right|_{\tilde{G}}$ avec $c_{0}=c_{a_{0}}$, et

$$
\sum_{w \in \mathfrak{B}} \int_{\tilde{G}} f_{X}(\tilde{g})\left\langle\tilde{\pi}_{1}(\tilde{g})(w), w^{\vee}\right\rangle d \tilde{g}=\left\langle\left. f_{X}\right|_{\tilde{G}}, \Theta_{\tilde{\pi}_{1}}\right\rangle .
$$

En définitive, on obtient que

$$
\left\langle f, \Theta_{\sigma_{1}}\right\rangle=s c_{0} c_{0}^{-1}\left\langle\left. f\right|_{\tilde{G}}, \Theta_{\tilde{\pi}_{1}}\right\rangle .
$$

Mais $s=\left|\epsilon_{a_{0}}\right|=\kappa c_{0}^{-1} c_{0}^{\prime}$ d'après (4.3), d'où l'égalité $\left\langle f, \Theta_{\sigma_{1}}\right\rangle=\kappa\left\langle\left. f_{X}\right|_{\tilde{G}}, \Theta_{\tilde{\pi}_{1}}\right\rangle$.

Puisque $G^{\prime}$ est ouvert dans $\tilde{G}$, on a

$$
\begin{aligned}
\left\langle\left. f_{X}\right|_{\tilde{G}}, \Theta_{\tilde{\pi}_{1}}\right\rangle & =\operatorname{trace}\left(\int_{\tilde{G}} f_{X}(\tilde{g}) \tilde{\pi}_{1}(\tilde{g}) d \tilde{g}\right) \\
& =\operatorname{trace}\left(\int_{G^{\prime}} C\left(\left.f_{X}\right|_{\tilde{G}}\right)\left(g^{\prime}\right) \pi_{1}^{\prime}\left(g^{\prime}\right) d g^{\prime}\right)=\left\langle C\left(\left.f_{X}\right|_{\tilde{G}}\right), \Theta_{\pi_{1}^{\prime}}\right\rangle .
\end{aligned}
$$

Ce qui achève la démonstration de la proposition.

Corollaire 4.5. Pour $f \in C_{\mathrm{c}}^{\infty}(H)$, on $a$

$$
\left\langle f, \Theta_{\sigma}\right\rangle=\kappa\left\langle\left. f\right|_{\tilde{G}}, \Theta_{\tilde{\pi}}\right\rangle=\kappa\left\langle C\left(\left.f\right|_{\tilde{G}}\right), \Theta_{\pi^{\prime}}\right\rangle .
$$

Démonstration. Puisque $\pi$ prolonge $\tilde{\pi}$, pour $f \in C_{\mathrm{c}}^{\infty}(H)$, on a

$$
\begin{aligned}
\operatorname{vol}(X, d h)\left\langle\left. f_{X}\right|_{\tilde{G}}, \Theta_{\tilde{\pi}}\right\rangle & =\operatorname{trace}\left(\sum_{i=1}^{s} \iint_{\tilde{G} \times K^{a_{0}}} f(\tilde{g}) \tilde{\pi}\left(h_{i} k \tilde{g} k^{-1} h_{i}^{-1}\right) d \tilde{g} d k\right) \\
& =\operatorname{trace}\left(\sum_{i=1}^{s} \int_{K^{a_{0}}} \pi\left(h_{i} k\right) \tilde{\pi}\left(\left.f\right|_{\tilde{G}}\right) \pi\left(k^{-1} h_{i}^{-1}\right) d k\right) \\
& =s c_{a_{0}}\left\langle\left. f\right|_{\tilde{G}}, \Theta_{\tilde{\pi}}\right\rangle .
\end{aligned}
$$

D'où le corollaire. 
Pour $a \in \mathbb{Z}_{\geq 0}$, on pose $e_{a}=c_{a}^{-1} \mathbf{1}_{K^{a}} \in C_{\mathrm{c}}^{\infty}(H)$ et l'on note $\Theta_{\pi, a}: H \rightarrow \mathbb{C}$ la fonction ( $K^{a}$-biinvariante) définie par

$\Theta_{\pi, a}(h)=\operatorname{trace}\left(\pi\left(e_{a}\right) \pi(h) \pi\left(e_{a}\right)\right)=c_{a}^{-1}\left(K^{a}: K^{a} \cap h^{-1} K^{a} h\right)^{-1} \operatorname{trace}\left(\pi\left(\mathbf{1}_{K^{a} h K^{a}}\right)\right)$.

Pour $f \in C_{\mathrm{c}}^{\infty}(H)$, on a $\left\langle f, \Theta_{\pi}\right\rangle=\left\langle f, \Theta_{\pi, a} d h\right\rangle$ pour tout $a \in \mathbb{Z}_{\geq 0}$ tel que $f \in$ $C_{\mathrm{c}}\left(K^{a} \backslash H / K^{a}\right)$. La distribution $\Theta_{\pi}$ est donc limite faible des distributions $\Theta_{\pi, a} d h$ quand $a$ tend vers $+\infty$.

Proposition 4.6. Soit $a_{1} \in \mathbb{Z}_{\geq a_{0}}$, et posons $b_{1}=b\left(\pi, a_{1}\right)$. Pour $f \in C_{\mathrm{c}}\left(K^{a_{1}} \backslash H / K^{a_{1}}\right)$ et $a \in \mathbb{Z}_{\geq b_{1}}$, on $a\left\langle f, \Theta_{\sigma}\right\rangle=\kappa \int_{\tilde{G}} f(\tilde{g}) \Theta_{\pi, a}(\tilde{g}) d \tilde{g}$.

Démonstration. Soient $f \in C_{\mathrm{c}}\left(K^{a_{1}} \backslash H / K^{a_{1}}\right)$ et $a \in \mathbb{Z}_{\geq b_{1}}$. D'après le lemme 4.1, on a

$$
\left\langle f, \Theta_{\sigma}\right\rangle=\operatorname{trace}\left(\sigma(f): V^{K^{a_{1}}} \rightarrow V^{K^{a_{1}}}\right)=\sum_{\chi \in \epsilon_{a}}\left\langle f, \Theta_{\pi \otimes \chi}\right\rangle
$$

avec $\left\langle f, \Theta_{\pi \otimes \chi}\right\rangle=\left\langle\chi f, \Theta_{\pi}\right\rangle$. Soit $X_{a} \subset H$ un système de représentants des classes $\tilde{G} K^{a} \backslash H$. On a

$$
\begin{aligned}
\left\langle f, \Theta_{\sigma}\right\rangle & =\sum_{\chi \in \epsilon_{a}} \operatorname{trace}\left(\int_{H}(\chi f)(h) \pi(h) d h\right) \\
& =\operatorname{trace}\left(\sum_{\chi \in \epsilon_{a}} \sum_{x \in X_{a}} \int_{\tilde{G} K^{a}}(\chi f)(h x) \pi(h x) d h\right) \\
& =\operatorname{trace}\left(\sum_{x \in X_{a}} \Xi_{a}(x) \int_{\tilde{G} K^{a}} f(h x) \pi(h x) d h\right)
\end{aligned}
$$

avec $\Xi_{a}(h)=\sum_{\chi \in \epsilon_{a}} \chi(h)(h \in H)$. Puisque $\Xi_{a}=\left|\epsilon_{a}\right| \mathbf{1}_{\tilde{G} K^{a}}$, on obtient que

$$
\left\langle f, \Theta_{\sigma}\right\rangle=\left|\epsilon_{a}\right| \cdot \operatorname{trace}\left(\int_{\tilde{G} K^{a}} f(h) \pi(h) d h\right) .
$$

Posons $c=c_{a}$ et $c^{\prime}=c_{a}^{\prime}$. Alors

$$
\begin{aligned}
\int_{\tilde{G} K^{a}} f(h) \pi(h) d h & =c^{-1} \iint_{K^{a} \times \tilde{G} K^{a}} f\left(k_{1} h\right) \pi\left(k_{1} h\right) d k_{1} d h \\
& =\left(c c^{\prime}\right)^{-1} \iiint_{K^{a} \times \tilde{G} \times K^{a}} f\left(k_{1} \tilde{g} k_{2}\right) \pi\left(k_{1} \tilde{g} k_{2}\right) d k_{1} d \tilde{g} d k_{2} \\
& =c^{2}\left(c c^{\prime}\right)^{-1} \int_{\tilde{G}} f(\tilde{g}) \pi\left(e_{a}\right) \pi(\tilde{g}) \pi\left(e_{a}\right) d \tilde{g} .
\end{aligned}
$$

Or $c c^{\prime-1}=\kappa \cdot\left|\epsilon_{a}\right|^{-1}$; voir (4.3). D'où l'on déduit que

$$
\left\langle f, \Theta_{\sigma}\right\rangle=\kappa \cdot \operatorname{trace}\left(\int_{\tilde{G}} f(\tilde{g}) \pi\left(e_{a}\right) \pi(\tilde{g}) \pi\left(e_{a}\right) d \tilde{g}\right)=\kappa \int_{\tilde{G}} f(\tilde{g}) \Theta_{\pi, a}(\tilde{g}) d \tilde{g} .
$$


Corollaire 4.7. Pour $f^{\prime} \in C_{\mathrm{c}}\left(K^{\prime a_{1}} \backslash G^{\prime} / K^{\prime a_{1}}\right)$ et $a \in \mathbb{Z}_{\geq b_{1}}$, on $a$

$$
\left\langle f^{\prime}, \Theta_{\pi^{\prime}}\right\rangle=\int_{G^{\prime}} f^{\prime}\left(g^{\prime}\right) \Theta_{\pi, a}\left(g^{\prime}\right) d g^{\prime} .
$$

Démonstration. Soient $f^{\prime} \in C_{\mathrm{c}}^{\infty}\left(K^{\prime a_{1}} \backslash G^{\prime} / K^{\prime a_{1}}\right)$ et $a \in \mathbb{Z}_{\geq b_{1}}$. Soit $f \in C_{\mathrm{c}}^{\infty}(H)$ la fonction prolongeant $f^{\prime}$ définie par

$$
f \in C_{\mathrm{c}}\left(K^{a_{1}} \backslash H / K^{a_{1}}\right) \quad \text { et } \quad \operatorname{Supp}(f)=K^{a_{1}} \operatorname{Supp}\left(f^{\prime}\right) K^{a_{1}} .
$$

D'après 4.5 et 4.6 , on a

$$
\left\langle{ }_{C}\left(\left.f\right|_{\tilde{G}}\right), \Theta_{\pi^{\prime}}\right\rangle=\kappa^{-1}\left\langle f, \Theta_{\sigma}\right\rangle=\int_{\tilde{G}} f(\tilde{g}) \Theta_{\pi, a}(\tilde{g}) d \tilde{g} .
$$

Puisque $\operatorname{Supp}\left(\left.f\right|_{\tilde{G}}\right) \subset G^{\prime}$, on a $C\left(\left.f\right|_{\tilde{G}}\right)=f^{\prime}$ et

$$
\int_{\tilde{G}} f(\tilde{g}) \Theta_{\pi, a}(\tilde{g}) d \tilde{g}=\int_{G^{\prime}} f^{\prime}\left(g^{\prime}\right) \Theta_{\pi, a}\left(g^{\prime}\right) d g^{\prime} .
$$

D'où le corollaire.

La distribution $\Theta_{\pi^{\prime}}$ est donc limite faible des distributions $\left.\Theta_{\pi, a}\right|_{G^{\prime}} d g^{\prime}$ quand $a$ tend vers $+\infty$. Les deux propositions suivantes sont naturellement impliquées par le corollaire 4.7, et seront utilisées dans la démonstration du théorème 4.12.

Proposition 4.8. On suppose la distribution $\Theta_{\pi}$ localement intégrable sur $H$. On choisit une fonction $\lambda_{\pi}: H \rightarrow \mathbb{C}$ localement intégrable par rapport à dh telle que $\Theta_{\pi}=\lambda_{\pi}$ dh. Soit $a_{1} \in \mathbb{Z}_{\geq a_{0}}$, et posons $b_{1}=b\left(\pi, a_{1}\right)$. Pour $f^{\prime} \in C_{\mathrm{c}}\left(K^{\prime a_{1}} \backslash G^{\prime} / K^{\prime a_{1}}\right)$, il existe une partie dense $\mathscr{V}_{\lambda_{\pi}, a_{1}}\left(f^{\prime}\right) \subset K^{b_{1}}$ telle que pour tout $k \in \mathscr{V}_{\lambda_{\pi}, a_{1}}\left(f^{\prime}\right)$, on a $\left\langle f^{\prime}, \Theta_{\pi^{\prime}}\right\rangle=\int_{G^{\prime}} f^{\prime}\left(g^{\prime}\right) \lambda_{\pi}\left(g^{\prime} k\right) d g^{\prime}$ (intégrale absolument convergente).

Démonstration. Pour $a \in \mathbb{Z}_{\geq 0}$, on a $\Theta_{\pi, a}(h)=c_{a}^{-2} \iint_{K^{a} \times K^{a}} \lambda_{\pi}\left(k_{1} h k_{2}\right) d k_{1} d k_{2}(h \in$ $H)$. Puisque $\Theta_{\pi}$ est une distribution $H$-invariante sur $H$, fixé un élément $y \in H$, on a $\lambda_{\pi}\left(y^{-1} h y\right)=\lambda_{\pi}(h)$ pour presque tout $h \in H$. Pour $a \in \mathbb{Z}_{\geq 0}$, on a donc aussi $\Theta_{\pi, a}(h)=c_{a}^{-1} \int_{K^{a}} \lambda_{\pi}(h k) d k(h \in H)$. D'après 4.7 , pour $f^{\prime} \in C_{\mathrm{c}}^{\infty}\left(G^{\prime}\right)$, on a

$$
\left\langle f^{\prime}, \Theta_{\pi^{\prime}}\right\rangle=\lim _{a \rightarrow+\infty} c_{a}^{-1} \iint_{G^{\prime} \times K^{a}} f^{\prime}\left(g^{\prime}\right) \lambda_{\pi}\left(g^{\prime} k\right) d g^{\prime} d k ;
$$

plus précisément, pour $f^{\prime} \in C_{\mathrm{c}}\left(K^{\prime a_{1}} \backslash G^{\prime} / K^{\prime a_{1}}\right)$ et $a \in \mathbb{Z}_{\geq b_{1}}$, on a

$$
\left\langle f^{\prime}, \Theta_{\pi^{\prime}}\right\rangle=c_{a}^{-1} \iint_{G^{\prime} \times K^{a}} f^{\prime}\left(g^{\prime}\right) \lambda_{\pi}\left(g^{\prime} k\right) d g^{\prime} d k .
$$

Soit $f^{\prime} \in C_{\mathrm{c}}\left(K^{\prime a_{1}} \backslash G^{\prime} / K^{\prime a_{1}}\right)$. Notons $f \in C_{\mathrm{c}}^{\infty}(H)$ la fonction prolongeant $f^{\prime}$ définie par $f \in C_{\mathrm{c}}\left(K^{a_{1}} \backslash H / K^{a_{1}}\right)$ et $\operatorname{Supp}(f)=K^{a_{1}} \operatorname{Supp}\left(f^{\prime}\right) K^{a_{1}}$. La fonction $H \rightarrow \mathbb{C}, h \mapsto f(h) \lambda_{\pi}(h)$ est intégrable par rapport à $d h$. Par conséquent (théorème de Fubini), pour presque tout $h \in H$, la fonction $\tilde{G} \rightarrow \mathbb{C}, \tilde{g} \mapsto f(\tilde{g} h) \lambda_{\pi}(\tilde{g} h)$ est 
intégrable par rapport à $d \tilde{g}$; et la fonction $\Phi_{f}: \tilde{G} \backslash H \rightarrow \mathbb{C}$, définie presque partout $\operatorname{par} \Phi_{f}(h)=\int_{\tilde{G}} f(\tilde{g} h) \lambda_{\pi}(\tilde{g} h) d \tilde{g}$, est intégrable par rapport à $d h / d \tilde{g}$.

L'ensemble $\epsilon$ des caractères de lisses de $\tilde{G} \backslash H$ est muni d' une structure de groupe topologique discret : c'est le dual de Pontryagin du groupe abélien compact $\tilde{G} \backslash H$. Notons $C_{0}(\tilde{G} \backslash H)$ (resp. $\left.L^{1}(\tilde{G} \backslash H)\right)$ l'espace des fonctions continues (resp. intégrables par rapport à $d h / d \tilde{g}) \tilde{G} \backslash H \rightarrow \mathbb{C}$. Soit $\Phi_{f}^{\vee}: \epsilon \rightarrow \mathbb{C}$ la transformée de Fourier de $\Phi_{f}$, définie par

$$
\Phi_{f}^{\vee}(\chi)=\int_{\tilde{G} \backslash H} \Phi_{f}(h) \chi\left(h^{-1}\right) \frac{d h}{d \tilde{g}} \quad(\chi \in \epsilon)
$$

(voir [Rudin 1962, 1.2.3]). Pour $\chi \in \epsilon$, on a (calcul facile) $\Phi_{f}^{\vee}(\chi)=\left\langle f, \Theta_{\pi \otimes \chi^{-1}}\right\rangle$. Par conséquent 4.1 $\Phi_{f}^{\vee}(\chi)=0$ pour tout $\chi \in \epsilon \backslash \epsilon_{b_{1}}$, donc $\sum_{\chi \in \epsilon}\left|\Phi_{f}^{\vee}(\chi)\right|<+\infty$. On peut donc définir la transformée de Fourier $\left(\Phi_{f}^{\vee}\right)^{\vee} \in C_{0}(\tilde{G} \backslash H)$ :

$$
\left(\Phi_{f}^{\vee}\right)^{\vee}(h)=\sum_{\chi \in \epsilon} \Phi_{f}^{\vee}(\chi) \chi\left(h^{-1}\right) \quad(h \in \tilde{G} \backslash H) .
$$

Puisque $\Phi_{f}^{\vee} \in L^{1}(\epsilon) \cap L^{1}(\tilde{G} \backslash H)^{\vee}$, on peut appliquer le théorème d'inversion de Fourier [Rudin 1962, 1.5.1] : $\left(\Phi_{f}^{\vee}\right)^{\vee} \in L^{1}(\tilde{G} \backslash H)$ et

$$
\Phi_{f}^{\vee}(\chi)=\vartheta^{-1} \int_{\tilde{G} \backslash H}\left(\Phi_{f}^{\vee}\right)^{\vee}(h) \chi(h) \frac{d h}{d \tilde{g}} \quad(\chi \in \epsilon)
$$

avec $\vartheta=\operatorname{vol}(\tilde{G} \backslash H, d h / d \tilde{g})=s \operatorname{vol}\left(K^{\prime a_{0}} \backslash K^{a_{0}}, d h / d \tilde{g}\right)=\left|\epsilon_{a_{0}}\right| c_{a_{0}}^{\prime} c_{a_{0}}^{-1}=\kappa$ (4.3). En d'autres termes, on a $\left(\Phi_{f}-\Psi\right)^{\vee}=0$ avec $\Psi(h)=\kappa^{-1}\left(\Phi_{f}^{\vee}\right)^{\vee}\left(h^{-1}\right)(h \in \tilde{G} \backslash H)$, ce qui implique (voir [Rudin 1962, 1.7.3/b]) que $\Phi_{f}(h)=\kappa^{-1}\left(\Phi_{f}^{\vee}\right)^{\vee}\left(h^{-1}\right)$ pour presque tout $h \in \tilde{G} \backslash H$. Puisque $\operatorname{Supp}\left(\Phi_{f}^{\vee}\right) \subset \epsilon_{b_{1}}$, on a

$$
\left(\Phi_{f}^{\vee}\right)^{\vee} \in C_{0}\left(\tilde{G} K^{b_{1}} \backslash H\right)=C\left(\tilde{G} K^{b_{1}} \backslash H\right) .
$$

Précisément, pour $h_{1} \in \tilde{G} \backslash H$, on a

$$
\begin{aligned}
\left(\Phi_{f}^{\vee}\right)^{\vee}\left(h_{1}\right) & =\sum_{\chi \in \epsilon_{b_{1}}} \int_{\tilde{G} \backslash H} \Phi_{f}\left(h h_{1}^{-1}\right) \chi\left(h^{-1}\right) \frac{d h}{d \tilde{g}} \\
& =\left|\epsilon_{b_{1}}\right| \int_{\tilde{G} \backslash \tilde{G} K^{b_{1}}} \Phi_{f}\left(h h_{1}^{-1}\right) \frac{d h}{d \tilde{g}} \\
& =\left|\epsilon_{b_{1}}\right| \int_{\tilde{G} K^{b_{1}}} f\left(h h_{1}^{-1}\right) \lambda_{\pi}\left(h h_{1}^{-1}\right) d h \\
& =\left|\epsilon_{b_{1}}\right| c_{b_{1}}^{-1} \iint_{\tilde{G} \times K^{b_{1}}} f\left(\tilde{g} k h_{1}^{-1}\right) \lambda_{\pi}\left(\tilde{g} k h_{1}^{-1}\right) d \tilde{g} d k .
\end{aligned}
$$


Puisque $\left|\epsilon_{b_{1}}\right| c_{b_{1}}^{\prime-1}=\kappa c_{b_{1}}^{-1}(4.3)$, pour $h_{1} \in \tilde{G} \backslash \tilde{G} K^{b_{1}}$, on a

$$
\left(\Phi_{f}^{\vee}\right)^{\vee}\left(h_{1}\right)=\kappa c_{b_{1}}^{-1} \iint_{\tilde{G} \times K^{b_{1}}} f(\tilde{g}) \lambda_{\pi}(\tilde{g} k) d \tilde{g} d k=\kappa\left\langle f^{\prime}, \Theta_{\pi^{\prime}}\right\rangle .
$$

Comme $\operatorname{Supp}\left(\left.f\right|_{\tilde{G}}\right) \subset G^{\prime}$, on obtient que

$$
\left\langle f^{\prime}, \Theta_{\pi^{\prime}}\right\rangle=\kappa^{-1}\left(\Phi_{f}^{\vee}\right)^{\vee}\left(h_{1}\right)=\int_{G^{\prime}} f^{\prime}\left(g^{\prime}\right) \lambda_{\pi}\left(g^{\prime} h_{1}\right) d g^{\prime}
$$

pour presque tout $h_{1} \in K^{b_{1}}$.

Proposition 4.9. Supposons la distribution $\Theta_{\pi}$ localement constante sur une partie ouverte y $\subset H$ telle que y $\cap G^{\prime} \neq \varnothing$. Alors la distribution $\Theta_{\pi^{\prime}}$ est localement constante sur y $\cap G^{\prime}$, et l'on a $\Theta_{\pi^{\prime}}\left(g^{\prime}\right)=\Theta_{\pi}\left(g^{\prime}\right)$ pour tout $g^{\prime} \in \mathcal{Y} \cap G^{\prime}$.

Démonstration. Posons $\mathscr{Y}^{\prime}=y \cap G^{\prime}$; c'est une partie ouverte (non vide) de $G^{\prime}$. Soit $f^{\prime} \in C_{\mathrm{c}}^{\infty}\left(\mathscr{Y}^{\prime}\right)$, et soit $a_{1} \in \mathbb{Z}_{\geq a_{0}}$ tel que $f \in C_{\mathrm{c}}\left(K^{\prime a_{1}} \backslash G^{\prime} / K^{\prime a_{1}}\right)$. Posons $b_{1}=b\left(\pi, a_{1}\right)$. Puisque $\operatorname{Supp}\left(f^{\prime}\right)$ est une partie compacte de $\mathcal{Y}^{\prime} \subset \mathcal{O}$, il existe un entier $a \geq b_{1}$ tel que $K^{a} \operatorname{Supp}\left(f^{\prime}\right) K^{a} \subset \mathcal{Y}$ et $\left.\Theta_{\pi}\right|_{K^{a}} \operatorname{Supp}\left(f^{\prime}\right) K^{a}$ est une fonction $K^{a}$-biinvariante. On a donc $\Theta_{\pi}\left(g^{\prime}\right)=\Theta_{\pi, a}\left(g^{\prime}\right)$ pour tout $g^{\prime} \in \operatorname{Supp}\left(f^{\prime}\right)$. On conclut grâce à 4.7.

Soit $Z$ le centre de $H$, et soit $\omega_{\pi} \in \epsilon(Z)$ le caractère central de $\pi$ défini (lemme de Schur) par $\pi(z)=\omega_{\pi}(z) \cdot \operatorname{Id}_{W}(z \in Z)$. Posons $Z^{a}=Z \cap K^{a}\left(a \in \mathbb{Z}_{\geq 0}\right)$, et notons $a_{\pi}$ le plus petit entier $a \geq 0$ tel que $\left.\omega_{\pi}\right|_{Z^{a}}=1$.

Remarque 4.10. Supposons la distribution $\Theta_{\pi}$ localement intégrable sur $H$, et choisissons une fonction $\lambda_{\pi}: H \rightarrow \mathbb{C}$ localement intégrable par rapport à $d h$ telle que $\Theta_{\pi}=\lambda_{\pi} d h$. Soient $f \in C_{\mathrm{c}}^{\infty}(H)$ et $z \in Z$. Notons $f^{z} \in C_{\mathrm{c}}^{\infty}(H)$ la fonction définie par $f^{z}(h)=f\left(h z^{-1}\right)(h \in H)$. On a donc $\left\langle f^{z}, \Theta_{\pi}\right\rangle=\int_{H} f(h) \lambda_{\pi}(h z) d h$. Par ailleurs, on a

$$
\left\langle f^{z}, \Theta_{\pi}\right\rangle=\operatorname{trace} \pi\left(f^{z}\right)=\omega_{\pi}(z) \text { trace } \pi(f)=\omega_{\pi}(z)\left\langle f, \Theta_{\pi}\right\rangle .
$$

Puisque l'égalité ci-dessus est vraie pour toute fonction $f \in C_{\mathrm{c}}^{\infty}(H)$, on obtient que $\lambda_{\pi}(h z)=\omega_{\pi}(z) \lambda_{\pi}(h)$ pour presque tout $h \in H$.

Remarque 4.11. Supposons la distribution $\Theta_{\pi}$ localement constante sur une partie ouverte $9 \subset H$. Pour $a \in \mathbb{Z}_{\geq 0}$, on a clairement $\Theta_{\pi, a}(h z)=\omega_{\pi}(z) \Theta_{\pi, a}(h)(h \in H$, $z \in Z$ ). On en déduit que la distribution $\Theta_{\pi}$ est localement constante sur $Z$ Yy, et que $\Theta_{\pi}(h z)=\omega_{\pi}(z) \Theta_{\pi}(h)$ pour tous $h \in$ Y et $z \in Z$.

On dit que la projection canonique $H \stackrel{p}{\longrightarrow} \tilde{G} \backslash H$ est scindée au-dessus d'un sous-groupe ouvert $U \subset \tilde{G} \backslash H$, s'il existe un morphisme de groupes topologiques (i.e., une application continue qui est un homomorphisme de groupes) $\sigma: U \rightarrow H$ tel que $p \circ \sigma=\operatorname{Id}_{U}$. Puisque $\tilde{G}=C G^{\prime}$ (produit direct) et $C \subset H$ est discret, la flèche $p$ est scindée au-dessus d'un sous-groupe ouvert de $\tilde{G} \backslash H$ si et seulement si 
la projection canonique $H \rightarrow G^{\prime} \backslash H$ est scindée au-dessus d'un sous-groupe ouvert de $G^{\prime} \backslash H$.

Thórème 4.12. Supposons la distribution $\Theta_{\pi}$ localement intégrable sur H. Si l'une des deux conditions suivantes est vérifiée:

(i) $\operatorname{vol}\left(G^{\prime} Z, d h\right) \neq \varnothing$ et la distribution $\Theta_{\pi}$ est localement constante sur une partie ouverte $\mathrm{y} \subset H$ telle que $\operatorname{vol}\left(G^{\prime} \backslash\left(\mathrm{Y} \cap G^{\prime}\right), d g^{\prime}\right)=0$,

(ii) la projection canonique $H \rightarrow G^{\prime} \backslash H$ est scindée au-dessus d'un sous-groupe ouvert de $G^{\prime} \backslash H$,

alors la distribution $\Theta_{\pi^{\prime}}$ est localement intégrable sur $G^{\prime}$.

Démonstration. Choisissons une fonction $\lambda_{\pi}: H \rightarrow \mathbb{C}$ localement intégrable par rapport à $d h$ telle que $\Theta_{\pi}=\lambda_{\pi} d h$.

Supposons vérifiée la condition (i). Soit $f^{\prime} \in C_{\mathrm{c}}^{\infty}\left(G^{\prime}\right)$, et soit $a_{1} \in \mathbb{Z}_{\geq a_{0}}$ tel que

$$
f^{\prime} \in C_{\mathrm{c}}\left(K^{\prime a_{1}} \backslash G^{\prime} / K^{\prime a_{1}}\right) .
$$

Posons $b_{1}=b\left(\pi, a_{1}\right)$ et $c_{1}=\max \left\{b_{1}, a_{\pi}\right\}$. On a $\operatorname{vol}\left(K^{\prime b_{1}} Z^{c_{1}}, d h\right) \neq 0$. Puisque $K^{\prime b_{1}} Z^{c_{1}}$ est contenu dans $K^{b_{1}}$ et que $f^{\prime} \in C_{\mathrm{c}}\left(G^{\prime} / K^{\prime b_{1}}\right)$, d'après la proposition 4.8, il existe un $z \in Z^{c_{1}}$ tel que $\left\langle f^{\prime}, \Theta_{\pi^{\prime}}\right\rangle=\int_{G^{\prime}} f^{\prime}\left(g^{\prime}\right) \lambda_{\pi}\left(g^{\prime} z\right) d g^{\prime}$ (intégrale absolument convergente). D'après la remarque 4.11 , pour tout $h \in \mathcal{Y}$, on a $\lambda_{\pi}(h z)=\lambda_{\pi}(h)$. Comme $\mathscr{Y} \cap G^{\prime}$ est dense dans $G^{\prime}$, on obtient que $\left\langle f^{\prime}, \Theta_{\pi^{\prime}}\right\rangle=\int_{G^{\prime}} f^{\prime}\left(g^{\prime}\right) \lambda_{\pi}\left(g^{\prime}\right) d g^{\prime}$ (intégrale absolument convergente). Cette égalité étant vérifiée pour toute fonction $f^{\prime} \in C_{\mathrm{c}}^{\infty}\left(G^{\prime}\right)$, la distribution $\Theta_{\pi^{\prime}}$ est localement intégrable sur $G^{\prime}$.

Supposons maintenant vérifiée la condition (ii); i.e., supposons qu'il existe un sous-groupe ouvert $U \subset \tilde{G} \backslash H$ et un morphisme de groupes topologiques $\sigma: U \rightarrow H$ tel que $p \circ \sigma=\operatorname{Id}_{U}$. Quitte à remplacer $U$ par un sous-groupe plus petit, on peut supposer que $\sigma(U) \subset K$. Pour $a \in \mathbb{Z}_{\geq 0}$, on pose $K_{\sigma}^{a}=K^{a} \sigma(U)$ (c'est un sousgroupe ouvert de $K), e_{a, \sigma}=\operatorname{vol}\left(K_{\sigma}^{a}, d k\right)^{-1} \mathbf{1}_{K_{\sigma}^{a}} \in C_{\mathrm{c}}^{\infty}(H)$, et l'on note $\Theta_{\pi, a, \sigma}$ : $H \rightarrow \mathbb{C}$ la fonction définie par $\Theta_{\pi, a, \sigma}(h)=\operatorname{trace}\left(\pi\left(e_{a, \sigma}\right) \pi(h) \pi\left(e_{a, \sigma}\right)\right)$.

Soit $a_{U}$ le plus petit entier $a \geq 0$ tel que $p\left(K^{a}\right) \subset U$. Posons $a_{0}^{*}=\max \left\{a_{0}, a_{U}\right\}$. Pour $a \in \mathbb{Z}_{\geq} a_{0}^{*}$, on a $K_{\sigma}^{a}=K^{\prime a} \sigma(U)$ (produit semidirect), et la restriction de $p$ à $K_{\sigma}^{a}$ induit une identification canonique $K^{\prime a} \backslash K_{\sigma}^{a}=U$. Soit $a_{1} \in \mathbb{Z}_{\geq a_{0}^{*}}$, et soit $b_{1}^{*}=b_{1}\left(\pi, a_{1}, \sigma\right)$ le plus petit entier $b \geq a_{1}$ tel que $\left\{\chi \in \epsilon: W^{K^{\prime a_{1}}}\left(\left.\chi\right|_{U} ^{-1}\right) \neq 0\right\} \subset \epsilon_{b}$. D'après la démonstration de 4.7 , pour $f^{\prime} \in C_{\mathrm{c}}^{\infty}\left(K^{\prime a_{1}} \backslash G^{\prime} / K^{\prime a_{1}}\right)$ et $a \in \mathbb{Z}_{\geq b_{1}^{*}}$, on a $\left\langle f^{\prime}, \Theta_{\pi^{\prime}}\right\rangle=\int_{G^{\prime}} f^{\prime}\left(g^{\prime}\right) \Theta_{\pi, a, \sigma}\left(g^{\prime}\right) d g^{\prime}$.

Pour $a \in \mathbb{Z}_{\geq 0}$, d'après le début de la démonstration de la proposition 4.8 , on a

$$
\Theta_{\pi, a, \sigma}(h)=\operatorname{vol}\left(K_{\sigma}^{a}, d k\right)^{-1} \int_{K_{\sigma}^{a}} \lambda_{\pi}(h k) d k \quad(h \in H) .
$$

Notons $d u$ la mesure de Haar sur $U$ déduite de $d h / d \tilde{g}$ par restriction. Pour $a \in$ $\mathbb{Z}_{\geq a_{0}^{*}}$ et $h \in H$, la fonction $K^{\prime a} \times U \rightarrow \mathbb{C},\left(k^{\prime}, u\right) \mapsto \lambda_{\pi}\left(h k^{\prime} \sigma(u)\right)$ est intégrable 
par rapport à $d k^{\prime} d u$, et l'on a $\Theta_{\pi, a, \sigma}(h)=c_{a}^{\prime-1} c_{U}^{-1} \iint_{K^{\prime a} \times U} \lambda_{\pi}\left(h k^{\prime} \sigma(u)\right) d k^{\prime} d u$ avec $c_{U}=\operatorname{vol}(U, d u)$. On en déduit (théorème de Fubini) que pour presque tout $g^{\prime} \in G^{\prime}$, la fonction $U \rightarrow \mathbb{C}, u \mapsto \lambda_{\pi}\left(g^{\prime} \sigma(u)\right)$ est intégrable par rapport à $d u$. Notons $\lambda_{\pi, \sigma}: G^{\prime} \rightarrow \mathbb{C}$ la fonction définie presque partout par

$$
\lambda_{\pi, \sigma}\left(g^{\prime}\right)=c_{U}^{-1} \int_{U} \lambda_{\pi}\left(g^{\prime} \sigma(u)\right) d u .
$$

Soit $f^{\prime} \in C_{\mathrm{c}}^{\infty}\left(G^{\prime}\right)$. Choisissons un $a_{1} \in \mathbb{Z}_{\geq a_{0}^{*}}$ tel que $f^{\prime} \in C_{\mathrm{c}}^{\infty}\left(K^{\prime a_{1}} \backslash G^{\prime} / K^{\prime a_{1}}\right)$ et posons $b_{1}^{*}=b(\pi, a, \sigma)$. Pour $a \in \mathbb{Z}_{\geq b_{1}^{*}}$, on a

$$
\begin{aligned}
\left\langle f^{\prime}, \Theta_{\pi^{\prime}}\right\rangle & =\int_{G^{\prime}} f^{\prime}\left(g^{\prime}\right) \Theta_{\pi, a, \sigma}\left(g^{\prime}\right) d g^{\prime} \\
& =c_{a}^{\prime-1} c_{U}^{-1} \int_{G^{\prime}} f^{\prime}\left(g^{\prime}\right)\left(\iint_{K^{\prime a} \times U} \lambda_{\pi}\left(g^{\prime} k^{\prime} \sigma(u)\right) d k^{\prime} d u\right) d g^{\prime} .
\end{aligned}
$$

La fonction $G^{\prime} \times K^{\prime a} \times U \rightarrow \mathbb{C},\left(g^{\prime}, k^{\prime}, u\right) \mapsto f^{\prime}\left(g^{\prime}\right) \Theta_{\pi}\left(g^{\prime} k^{\prime} \sigma(u)\right.$ est intégrable par rapport à $d g^{\prime} d k^{\prime} d u$. On peut donc inverser les signes $\int_{G^{\prime}}$ et $\int_{K^{\prime a}}$ (théorème de Fubini). Puisque $f^{\prime}\left(g^{\prime} k^{\prime-1}\right)=f^{\prime}\left(g^{\prime}\right)\left(g^{\prime} \in G^{\prime}, k^{\prime} \in K^{\prime a}\right)$, on obtient que la fonction $G^{\prime} \times U \rightarrow \mathbb{C}, g^{\prime} \mapsto f^{\prime}\left(g^{\prime}\right) \Theta_{\pi}\left(g^{\prime} \sigma(u)\right)$ est intégrable par rapport à $d g^{\prime} d u$, et que

$$
\left\langle f^{\prime}, \Theta_{\pi^{\prime}}\right\rangle=\int_{G^{\prime}} f^{\prime}\left(g^{\prime}\right) \lambda_{\pi, \sigma}\left(g^{\prime}\right) d g^{\prime}
$$

(intégrale absolument convergente). La fonction $G^{\prime} \rightarrow \mathbb{C}, g^{\prime} \mapsto \lambda_{\pi, \sigma}\left(g^{\prime}\right)$ est donc localement intégrable par rapport à $d g^{\prime}$, et la distribution $\Theta_{\pi^{\prime}}$ est localement intégrable sur $G^{\prime}$.

Remarque 4.13. Supposons la distribution $\Theta_{\pi}$ localement intégrable sur $H$. Supposons aussi que la projection canonique $H \rightarrow \tilde{G} \backslash H$ est scindée au-dessus d'un sous-groupe ouvert $U$ de $\tilde{G} \backslash H$. Soit $\sigma: U \rightarrow H$ un morphisme de groupes topologiques tels que $p \circ \sigma=\operatorname{Id}_{U}$. Puisque $U$ est fermé dans le groupe compact $\tilde{G} \backslash H, \sigma(U)$ est un sous-groupe compact de $H$. Par conséquent $\sigma(U)$ est contenu dans un sous-groupe ouvert compact $J$ de $H$, et quitte à remplacer $K$ par $J$, on peut supposer que $\sigma(U) \subset K$. Choisissons une fonction $\lambda_{\pi}: H \rightarrow \mathbb{C}$ localement intégrable par rapport à $d h$ telle que $\Theta_{\pi}=\lambda_{\pi} d h$. D'après la démonstration de 4.12 , pour presque tout $g^{\prime} \in G^{\prime}$, la fonction $U \rightarrow \mathbb{C}, u \mapsto \lambda_{\pi}\left(g^{\prime} \sigma(u)\right)$ est intégrable par rapport à $d u=\left.(d h / d \tilde{g})\right|_{U}$; la fonction $\lambda_{\pi, \sigma}: G^{\prime} \rightarrow \mathbb{C}$ définie presque partout par $\lambda_{\pi, \sigma}\left(g^{\prime}\right)=\Theta_{\pi^{\prime}}\left(g^{\prime}\right)=\operatorname{vol}(U, d u)^{-1} \int_{U} \lambda_{\pi}\left(g^{\prime} u\right) d u$, est localement intégrable par rapport à $d g^{\prime}$; et l'on a $\Theta_{\pi^{\prime}}=\lambda_{\pi, \sigma} d g^{\prime}$.

Supposons de plus que la distribution $\Theta_{\pi}$ est localement constante sur une partie ouverte $\mathscr{Y} \subset H$ telle que $\mathscr{y} \cap G^{\prime} \neq \varnothing$. Alors d'après la proposition 4.9, pour tout $g^{\prime} \in \mathcal{Y} \cap G^{\prime}$, on a $\Theta_{\pi}\left(g^{\prime}\right)=\lambda_{\pi, \sigma}\left(g^{\prime}\right)$. 
Corollaire 4.14. Supposons la distribution $\Theta_{\pi}$ localement intégrable sur $H$, localement constante sur une partie ouverte $\mathscr{y} \subset H$ telle que $\operatorname{vol}\left(G^{\prime} \backslash\left(\mathrm{Y}_{\cap} \cap G^{\prime}\right), d g^{\prime}\right)=0$. Si $\operatorname{vol}\left(G^{\prime} Z, d h\right) \neq 0$ ou si la projection canonique $H \rightarrow G^{\prime} \backslash H$ est scindée audessus d'un sous-groupe ouvert de $G^{\prime} \backslash H$, alors la fonction $\left.\Theta_{\pi}\right|_{G^{\prime}}$ est localement intégrable par rapport à $d g^{\prime}$, et pour toute fonction $f^{\prime} \in C_{\mathrm{c}}^{\infty}\left(G^{\prime}\right)$, on a $\left\langle f^{\prime}, \Theta_{\pi^{\prime}}\right\rangle=$ $\int_{G^{\prime}} f^{\prime}\left(g^{\prime}\right) \Theta_{\pi}\left(g^{\prime}\right) d g^{\prime}$.

\section{Intégrabilité locale des caractères de $G^{\prime}(F)$}

Soit $\boldsymbol{G}$ un groupe linéaire algébrique réductif connexe défini sur $F$, et soit $\boldsymbol{G}^{\prime}$ son groupe dérivé. Soit $\boldsymbol{C}$ le tore central $F$-déployé maximal de $\boldsymbol{G}$, et soit $\boldsymbol{C}(\varpi)$ le sous-groupe de $\boldsymbol{C}(F)=\operatorname{Hom}\left(\mathrm{X}^{*}(\boldsymbol{C}), F^{\times}\right)$défini par $\boldsymbol{C}(\varpi)=\operatorname{Hom}\left(\mathrm{X}^{*}(\boldsymbol{C}),\langle\varpi\rangle\right)$; où $\mathrm{X}^{*}(\boldsymbol{C})$ désigne le $\mathbb{Z}$-module libre formé des caractères algébriques de $\boldsymbol{C}$. Posons ${ }_{\varpi} \boldsymbol{G}^{\prime}(F)=\boldsymbol{C}(\varpi) \boldsymbol{G}^{\prime}(F)$ (produit direct) ; c'est un sous-groupe fermé cocompact $\boldsymbol{G}(F)$. Remarquons que pour tout sous-groupe compact $J \subset \boldsymbol{G}(F)$, on a $J \cap \boldsymbol{C}(\varpi)=0$. Soit $H \subset \boldsymbol{G}(F)$ un sous-groupe ouvert contenant ${ }_{\varpi} \boldsymbol{G}^{\prime}(F)$. Le triplet $\left(H, \boldsymbol{G}^{\prime}(F), \boldsymbol{C}(\varpi)\right)$ vérifie toutes les conditions imposées au triplet $\left(H, G^{\prime}, C\right)$ dans la section 4 .

Soit $\left(\pi^{\prime}, W\right)$ une représentation complexe lisse irréductible, donc admissible, de $\boldsymbol{G}^{\prime}(F)$. Notons $(\tilde{\pi}, W)$ la représentation de $\tilde{G}={ }_{\omega} \boldsymbol{G}^{\prime}(F)$ définie par $\tilde{\pi}\left(z g^{\prime}\right)=\tilde{\pi}\left(g^{\prime}\right)$ pour tous $z \in \boldsymbol{C}(\varpi)$ et $g^{\prime} \in \boldsymbol{G}^{\prime}(F)$. On sait que toute représentation complexe lisse de type fini de $\boldsymbol{G}^{\prime}(F)$ est un $\mathbb{C}\left[\boldsymbol{G}^{\prime}(F)\right]$-module nœthérien. On en déduit que toute représentation complexe lisse de type fini de $\tilde{G}$ est un $\mathbb{C}[\tilde{G}]$-module nœthérien. On peut donc appliquer le corollaire 4.6 de [Henniart 2001] : la représentation $(\tilde{\pi}, W)$ de $\tilde{G}$ s'étend en une représentation lisse $(\pi, W)$ d'un sous-groupe ouvert d'indice fini $H \subset \boldsymbol{G}(F)$. Par construction, $\pi$ est admissible et irréductible. Puisque $H$ contient $\boldsymbol{G}^{\prime}(F), H$ est distingué dans $\boldsymbol{G}(F)$.

Soient $d g$ et $d g^{\prime}$ des mesures de Haar respectivement sur $\boldsymbol{G}(F)$ et $\boldsymbol{G}^{\prime}(F)$, et posons $d h=\left.d g\right|_{H}$. Pour toute représentation complexe lisse admissible $\pi_{1}$ de $H$ (resp. $\pi_{1}^{\prime}$ de $\left.\boldsymbol{G}^{\prime}(F)\right)$, on pose $\Theta_{\pi_{1}}=\operatorname{trace}\left(\pi_{1} d h\right) \in \mathscr{D}(H)$ et $\Theta_{\pi_{1}^{\prime}}=\operatorname{trace}\left(\pi_{1}^{\prime} d g^{\prime}\right) \in$ $\mathscr{D}\left(\boldsymbol{G}^{\prime}(F)\right)$.

Soit $\boldsymbol{G}(F)_{\text {sr }}$ l'ensemble des éléments semisimples réguliers de $\boldsymbol{G}(F)$. Posons $H_{\mathrm{sr}}=\boldsymbol{G}(F)_{\mathrm{sr}} \cap H$ et $\boldsymbol{G}^{\prime}(F)_{\mathrm{sr}}=\boldsymbol{G}(F)_{\mathrm{sr}} \cap \boldsymbol{G}^{\prime}(F)$.

Le résultat suivant est une variante de [Harish-Chandra 1980, 4].

Proposition 5.1. Pour toute représentation lisse irréductible $\pi_{1}$ de $H$, la distribution $\Theta_{\pi_{1}}$ est localement constante sur $H_{\mathrm{sr}}$.

Démonstration. Elle est identique à celle de [Harish-Chandra 1980]. Soient $(\boldsymbol{P}, \boldsymbol{A})$ une $F$-paire parabolique minimale dans $\boldsymbol{G}$, et $\mathscr{K}$ un sous-groupe compact maximal spécial de $\boldsymbol{G}(F)$ en bonne position par rapport à $(\boldsymbol{P}(F), \boldsymbol{A}(F))$; i.e. tel que $\boldsymbol{G}(F)=$ $\mathscr{H} \boldsymbol{P}(F)$ et $\mathscr{K} \cap \boldsymbol{P}(F)=(\mathscr{K} \cap \boldsymbol{M}(F))(\mathscr{K} \cap \boldsymbol{U}(F))$ où $\boldsymbol{M}$ et $\boldsymbol{U}$ désignent respectivement 
le centralisateur de $\boldsymbol{A}$ dans $\boldsymbol{G}$ et le radical unipotent de $\boldsymbol{P}$. On a la décomposition d'Iwasawa $\boldsymbol{G}^{\prime}(F)=\mathscr{K ^ { \prime }} \boldsymbol{P}^{\prime}(F)$ avec $\mathscr{K}^{\prime}=\mathscr{K} \cap \boldsymbol{G}^{\prime}(F)$ et $\boldsymbol{P}^{\prime}=\boldsymbol{P} \cap \boldsymbol{G}^{\prime}$. Puisque $\boldsymbol{G}(F)=\boldsymbol{G}^{\prime}(F) \boldsymbol{M}(F)$, on a aussi $\boldsymbol{G}(F)=\mathscr{K ^ { \prime }} \boldsymbol{P}(F)$, d'où l'on déduit que

$$
\mathscr{K}=\mathscr{K} \mathcal{K}^{\prime}(\mathscr{K} \cap \boldsymbol{P}(F))=\mathscr{K}^{\prime}(\mathscr{K} \cap \boldsymbol{M}(F))
$$

et que $H=\mathscr{K}_{H} P_{H}$ avec $\mathscr{K}_{H}=\mathscr{K} \cap H$ et $P_{H}=\boldsymbol{P}(F) \cap H$.

Soit $M^{+}$le sous-ensemble de $\boldsymbol{M}(F)$ défini comme en [Harish-Chandra 1980, 4], et soit $\Omega \subset \mathscr{K} \cap \boldsymbol{M}(F)$ un système (fini) de représentants des classes $\mathscr{K}_{H} \backslash \mathscr{K}$. On a la décomposition de Cartan $\boldsymbol{G}(F)=\mathscr{K} M^{+} \mathscr{K}$, d'où l'on déduit que $H=$ $\mathscr{K}_{H}\left(\Omega M^{+} \Omega\right)_{H} \mathscr{K}_{H}$ avec $\left(\Omega M^{+} \Omega\right)_{H}=\Omega M^{+} \Omega \cap H$. Soit $\mathscr{K}_{0} \subset \mathscr{K}_{H}$ un sous-groupe ouvert distingué tel que $\left(V_{\pi_{1}}\right)^{\mathscr{K}_{0}} \neq 0$ où $V_{\pi_{1}}$ désigne l'espace de $\pi_{1}$. D'après [Harish-Chandra 1980, 4], il existe un sous-groupe ouvert compact $\mathscr{P}_{0} \subset \boldsymbol{P}(F)$, que l'on peut choisir contenu dans $P_{H}$, tel que $m^{-1} \mathscr{P}_{0} m \subset \mathscr{K}_{0}$ pour tout $m \in M^{+}$. Quitte à remplacer $\mathscr{P}_{0} \operatorname{par} \bigcap_{x \in \Omega} x \mathscr{P}_{0} x^{-1}$, on peut supposer que $m^{-1} \mathscr{P}_{0} m \subset \mathscr{K}_{0}$ pour tout $m \in \Omega M^{+} \Omega$. Comme dans [Harish-Chandra 1980], on montre alors que pour $x \in H_{\mathrm{sr}}$, l'opérateur $T_{x}=\operatorname{vol}\left(\mathscr{K}_{H}, d h\right)^{-1} \int_{\mathscr{K}_{H}} \pi_{1}\left(h x h^{-1}\right) d h$ est de rang fini, et que l'application $H_{\mathrm{sr}} \rightarrow \operatorname{End}\left(V_{\pi_{1}}\right), x \mapsto T_{x}$ est localement constante; ce qui implique (voir [Harish-Chandra 1980, 4, corollary of theorem 2]) que $\Theta_{\pi_{1}}$ coïncide sur $H_{\text {sr }}$ avec l'application localement constante $x \mapsto \operatorname{trace}\left(T_{x}\right)$.

D'après [Harish-Chandra 1980], la distribution $\Theta_{\pi^{\prime}}$ est une fonction localement constante sur l'ensemble $\boldsymbol{G}^{\prime}(F)_{\mathrm{sr}}$. D'après la proposition 4.9 , on a donc le

Corollaire 5.2. On $\left.a \Theta_{\pi^{\prime}}\right|_{\boldsymbol{G}^{\prime}(F)_{\mathrm{sr}}}=\left.\Theta_{\pi}\right|_{\boldsymbol{G}^{\prime}(F)_{\mathrm{sr}}}$.

Soit $\boldsymbol{Z}$ le centre de $\boldsymbol{G}$. Posons $Z_{H}=\boldsymbol{Z}(F) \cap H$; c'est le centre de $H$. Puisque $H$ est un sous-groupe ouvert d'indice fini de $\boldsymbol{G}(F)$, on a

$-\operatorname{vol}\left(\boldsymbol{G}^{\prime}(F) Z_{H}, d h\right) \neq 0$ si et seulement $\operatorname{si} \operatorname{vol}\left(\boldsymbol{G}^{\prime}(F) \boldsymbol{Z}(F), d g\right) \neq 0$,

- la projection canonique $H \rightarrow \boldsymbol{G}^{\prime}(F) \backslash H$ est scindée au-dessus d'un sousgroupe ouvert de $\boldsymbol{G}^{\prime}(F) \backslash H$ si et seulement si la projection canonique $\boldsymbol{G}(F) \rightarrow$ $\boldsymbol{G}^{\prime}(F) \backslash \boldsymbol{G}(F)$ est scindée au-dessus d'un sous-groupe ouvert de $\boldsymbol{G}^{\prime}(F) \backslash \boldsymbol{G}(F)$.

Soit $p \geq 0$ la caractéristique de $F$. Si $p=0$, ou si $p$ est $>0$ et "suffisamment grand" par rapport au rang de $\boldsymbol{G}$, alors les deux critères qui nous intéressent sont vérifiés : 1'application produit $\boldsymbol{G}^{\prime}(F) \times \boldsymbol{Z}(F) \rightarrow \boldsymbol{G}(F)$ est ouverte, de noyau fini ; par conséquent $\operatorname{vol}\left(\boldsymbol{G}^{\prime}(F) \boldsymbol{Z}(F), d h\right) \neq 0$, et pour tout sous-groupe ouvert $U_{Z} \subset$ $\boldsymbol{Z}(F)$ tel que $U_{Z} \cap \boldsymbol{G}^{\prime}(F)=\{1\}$, la projection canonique $\boldsymbol{G}(F) \rightarrow \boldsymbol{G}^{\prime}(F) \backslash \boldsymbol{G}(F)$ est scindée au-dessus de $\boldsymbol{G}^{\prime}(F) \backslash \boldsymbol{G}^{\prime}(F) U_{Z}$. Dans le cas général, il se peut que $\operatorname{vol}\left(\boldsymbol{G}^{\prime}(F) \boldsymbol{Z}(F), d g\right)=0$ (cf. par exemple la section 6), mais le second critère est toujours vérifié :

Lemme 5.3. La projection canonique $\boldsymbol{G}(F) \rightarrow \boldsymbol{G}^{\prime}(F) \backslash \boldsymbol{G}(F)$ est scindée au-dessus d'un sous-groupe ouvert de $\boldsymbol{G}^{\prime}(F) \backslash \boldsymbol{G}(F)$. 
Démonstration (P. Gille). Soit $1 \rightarrow \boldsymbol{T}_{1} \stackrel{\iota}{\rightarrow} \boldsymbol{T}_{2} \stackrel{\pi}{\rightarrow} \boldsymbol{T}_{3} \rightarrow 1$ une suite exacte de $F$ tores. Choisissons une extension galoisienne finie $E / F$ déployant $\boldsymbol{T}_{1}$ et $\boldsymbol{T}_{3}$ (alors $E$ déploie $\left.\boldsymbol{T}_{2}\right)$, et posons $\Gamma=\operatorname{Gal}(E / F)$. Pour $i=1,2,3$, on note $X_{i}=\mathrm{X}^{*}\left(\boldsymbol{T}_{i}\right)$ le groupe des caractères algébriques de $\boldsymbol{T}_{i}$. D'où une suite exacte de $\Gamma$-modules $0 \rightarrow X_{3} \stackrel{\pi^{*}}{\rightarrow} X_{2} \stackrel{\iota^{*}}{\rightarrow} X_{1} \rightarrow 0$, laquelle définit un élément de $\operatorname{Ext}_{\Gamma}^{1}\left(X_{1}, X_{3}\right)$. Puisque $|\Gamma| \cdot \operatorname{Ext}_{\Gamma}^{1}\left(X_{1}, X_{3}\right)=0$, la suite exacte de $\Gamma$-modules

$$
1 \rightarrow X_{3} \stackrel{\tilde{\pi}^{*}}{\rightarrow} \tilde{X}_{2} \stackrel{\tilde{\iota}^{*}}{\rightarrow} X_{1} \rightarrow 1
$$

définie par

- $\tilde{X}_{2}=\operatorname{ker}\left\{X_{1} \times X_{2} \rightarrow X_{1},(x, y) \mapsto|\Gamma| x+\iota^{*}(y)\right\}$,

- $\tilde{\pi}^{*}(y)=\left(0, \pi^{*}(y)\right)$ pour tout $y \in X_{3}$,

- $\tilde{\iota}^{*}(x, y)=x$ pour tout $(x, y) \in \tilde{X}_{2} \subset X_{1} \times X_{2}$,

est scindée. De plus, le diagramme de $\Gamma$-modules

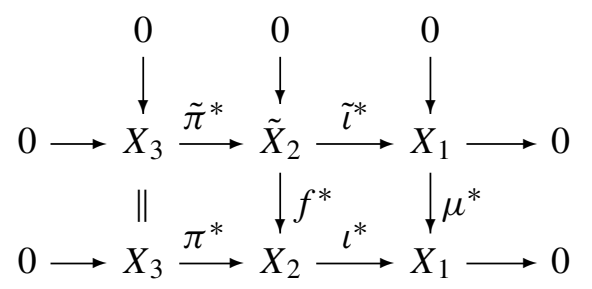

est commutatif; où $f^{*}$ désigne la projection sur le second facteur, et $\mu^{*}$ la multiplication par $|\Gamma|$. Le conoyau de $f^{*}$ s'identifie naturellement (i.e., via $\iota^{*}$ ) à un sous-groupe de $X_{1} / \mu^{*}\left(X_{1}\right)$; c'est donc un groupe fini. Par dualité, on obtient le diagramme commutatif exact de $F$-tores suivant :

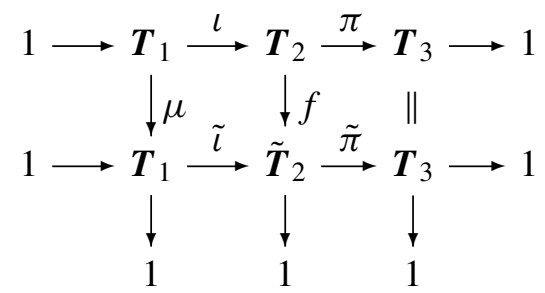

où $\tilde{\boldsymbol{T}}_{2}$ désigne le $F$-tore $E$-déployé ayant pour groupe des caractères le $\Gamma$-module $\tilde{X}_{2}$. Soit $\tilde{s}: \boldsymbol{T}_{3} \rightarrow \tilde{\boldsymbol{T}}_{2}$ un morphisme de $F$-tores tel que $\tilde{\pi} \circ \tilde{s}=\mathrm{id}_{\boldsymbol{T}_{3}}$ (un tel $\tilde{s}$ existe, cf. plus haut). Puisque ker $f$ est un $F$-groupe multiplicatif fini, il existe un sousgroupe ouvert $U_{2} \subset \boldsymbol{T}_{2}(F)$ tel que $f$ induit un homéomorphisme (pour la topologie $\varpi$-adique) $f^{\prime}: U_{2} \rightarrow f\left(U_{2}\right)$. Comme $f\left(U_{2}\right)$ est ouvert dans $\tilde{\boldsymbol{T}}_{2}(F)$, il existe un sous-groupe ouvert $U_{3} \subset \boldsymbol{T}_{3}(F)$, que l'on peut supposer contenu dans $\pi\left(\boldsymbol{T}_{2}(F)\right) \cap$ $\tilde{\pi}\left(\tilde{\boldsymbol{T}}_{2}(F)\right)$, tel que $\tilde{s}\left(U_{3}\right) \subset f\left(U_{2}\right)$. Soit $s: U_{3} \rightarrow \boldsymbol{T}_{2}(F)$ l'homomorphisme de groupes défini par $s(u)=\left.f^{\prime-1} \circ \tilde{s}\right|_{U_{3}}$; il est continu et scinde la suite exacte $1 \rightarrow$ $\boldsymbol{T}_{1}(F) \rightarrow \boldsymbol{T}_{2}(F) \rightarrow \pi\left(\boldsymbol{T}_{2}(F)\right) \rightarrow 1$ au-dessus de $U_{3}$. 
Le quotient géométrique $p: \boldsymbol{G} \rightarrow \boldsymbol{G}^{\prime} \backslash \boldsymbol{G}$ existe sur $F$; plus précisément, $\boldsymbol{G}^{\prime} \backslash \boldsymbol{G}$ est un $F$-tore et $p$ est un morphisme de $F$-groupes algébriques. Soit $\boldsymbol{T}$ un tore maximal de $\boldsymbol{G}$ défini sur $F$. Alors $\boldsymbol{T}^{\prime}=\boldsymbol{T} \cap \boldsymbol{G}^{\prime}$ est un tore maximal de $\boldsymbol{G}^{\prime}$ défini sur $F$, et $p$ induit une suite exacte de $F$-tores $1 \rightarrow \boldsymbol{T}^{\prime} \rightarrow \boldsymbol{T} \rightarrow \boldsymbol{G}^{\prime} \backslash \boldsymbol{G} \rightarrow 1$. D'où le lemme.

D'après le corollaire 4.14, la proposition 5.1 et le lemme 5.3, on a :

Thórème 5.4. Supposons la distribution $\Theta_{\pi}$ localement intégrable sur H. Alors la distribution $\Theta_{\pi^{\prime}}$ est localement intégrable sur $\boldsymbol{G}^{\prime}(F)$; plus précisément, la fonction $\left.\Theta_{\pi}\right|_{\boldsymbol{G}^{\prime}(F)}$ est localement intégrable par rapport à $d g^{\prime}$, et pour toute fonction $f^{\prime} \in$ $C_{\mathrm{c}}^{\infty}\left(\boldsymbol{G}^{\prime}(F)\right)$, on $a\left\langle f^{\prime}, \Theta_{\pi^{\prime}}\right\rangle=\int_{\boldsymbol{G}^{\prime}(F)} f^{\prime}\left(g^{\prime}\right) \Theta_{\pi}\left(g^{\prime}\right) d g^{\prime}$.

\section{Intégrabilité locale des caractères de $\mathrm{SL}_{n}(D)$}

Revenons à la situation qui nous intéresse, celle des sections 1 à $3: G=$ $\operatorname{Aut}_{D}(V), G^{\prime}=\operatorname{ker}\left\{\operatorname{det}^{\prime}: G \rightarrow F^{\times}\right\}$et $\tilde{G}={ }_{\varpi} G^{\prime}$. La norme réduite $\operatorname{det}^{\prime}: G \rightarrow F^{\times}$ induit par passage aux quotients un isomorphisme de groupes

$$
G^{\prime} Z \backslash G \rightarrow\left(F^{\times}\right)^{n} \backslash F^{\times} \simeq\left(\mathfrak{o}_{F}^{\times}\right)^{n} \backslash \mathfrak{o}_{F}^{\times} \times \mathbb{Z} / n \mathbb{Z} .
$$

Si $p(=\operatorname{car}(F))=0$, ou si $p>0$ et $(n, p)=1$, alors le groupe $G^{\prime} Z \backslash G$ est fini et $G^{\prime} Z$ est un sous-groupe ouvert de $G$. Si $p>0$ et $p$ divise $n$, alors $\operatorname{vol}\left(G^{\prime} Z, d g\right)=$ 0 . Pour $x \in F^{\times}$, notons $\mu(x) \in G$ l'élément défini par $\mu(x)\left(e_{1}\right)=e_{1} \cdot x$ et $\mu(x)\left(e_{i}\right)=e_{i}(i=2, \ldots, n)$; on rappelle que $\left(e_{1}, \ldots, e_{n}\right)$ est une $D$-base de $V)$. Alors l'homomorphisme de groupes $\sigma=\mu \circ \operatorname{det}^{\prime}: G^{\prime} \backslash G \rightarrow G$ est continu et vérifie $\sigma(x)\left(\bmod G^{\prime}\right)=x^{d}\left(x \in G^{\prime} \backslash G\right)$. En particulier, si $D=F$, alors la projection canonique $G \rightarrow G^{\prime} \backslash G$ est non seulement scindée au-dessus d'un sousgroupe ouvert de $G^{\prime} \backslash G$ (lemme 5.3), mais elle est scindée au-dessus de $G^{\prime} \backslash G$.

Soient $d g$ et $d g^{\prime}$ des mesures de Haar respectivement sur $G$ et $G^{\prime}$ (le sous-groupe $H \subset G$ n'étant plus fixé, on abandonne les normalisations de la section 1). Pour tout sous-groupe ouvert distingué $H \subset G$, on pose $d h=\left.d g\right|_{H}$. Pour toute représentation complexe lisse admissible $\pi^{\prime}$ de $G^{\prime}$, on note $\Theta_{\pi^{\prime}} \in \mathscr{D}\left(G^{\prime}\right)$ la distribution $\operatorname{trace}\left(\pi^{\prime} d g^{\prime}\right)$.

D'après le corollaire 3.9 , la proposition 4.9 et le lemme 5.3 , on a :

Thórème 6.1. Soit $\pi^{\prime} \in \epsilon\left(G^{\prime}\right)$. la distribution $\Theta_{\pi^{\prime}}$ est localement intégrable sur $G^{\prime}$, localement constante sur $G_{\mathrm{r}}^{\prime}$.

Soit $\left(\pi^{\prime}, W\right)$ une représentation complexe lisse irréductible de $G^{\prime}$. Choisissons un sous-groupe ouvert $H \subset G$ contenant $\tilde{G}$ et une représentation lisse $(\pi, W)$ de $H$ prolongeant $\pi^{\prime}$ et telle que $\pi\left(\varpi^{i} h\right)=\pi(h)(i \in \mathbb{Z}, h \in H)$. D'après le lemme 5.3, on a $\Theta_{\pi^{\prime}}\left(g^{\prime}\right)=\Theta_{\pi}\left(g^{\prime}\right)$ pour presque tout $g^{\prime} \in G^{\prime}$ (resp. pour tout $g^{\prime} \in G_{\mathrm{r}}^{\prime}$ ). En particulier, la distribution $\Theta_{\pi^{\prime}}$ est $H$-invariante. Il suffit donc de décrire $\Theta_{\pi^{\prime}}$ 
au voisinage des éléments $G$-fermés de $G^{\prime}$. Soit $x$ un tel élément. Reprenons les notations introduites dans le paragraphe "Réduction aux éléments $G$-purs de $H$ (descente parabolique)" (page 101). D'après le corollaire 3.9, il existe un entier $m>0$ tel que $\mathfrak{A}_{\natural}^{m} \varrho \subset \mathfrak{A}^{1 / d}$ et $1+\mathfrak{A}_{\natural}^{m} \varrho \subset H$, et des constantes $c_{n}\left(\pi^{\prime}\right)\left(n \in \mathcal{N}_{H, \sharp}\right)$ tels que

$$
\Theta_{\pi^{\prime}}((1+u \varrho) x)=\sum_{n \in \mathcal{N}_{H, \sharp}} c_{n}\left(\pi^{\prime}\right) \Theta_{\sharp, n}^{\vee}(u)
$$

pour tout $u \in \mathfrak{A}_{\natural}^{m} \cap B^{\prime}$ tel que $1+u \varrho \in G^{\prime}$; où $\mathcal{N}_{H, \natural}$ désigne un système (fini) de représentants dans $B$ des $H_{x}$-orbites nilpotentes de $B, \Theta_{\natural, n}^{\vee}$ la transformée de Fourier de la distribution $\Theta_{\natural, n} \in J_{H_{x}}(B)$ définie (via le choix de mesures de Haar $d h_{\natural}$ et $d h_{\natural, n}$ respectivement sur $H_{x}$ et $\left.\left(H_{x}\right)_{n}\right)$ comme dans la section 3, et $B^{\prime}$ l'ensemble des éléments semisimples réguliers de $B$. Les fonctions $\left.\Theta_{\natural, n}^{\vee}\right|_{B^{\prime}}$ sont en général difficilement calculable (sauf si $H=G$, auquel cas l'on dispose d'une très jolie formule, [Lemaire 2004, 1.9]). On aimerait donc les remplacer par d'autres fonctions sur $B^{\prime}$, plus faciles à manipuler : les transformées de Fourier des $B^{\times}$intégrales orbitales nilpotentes sur $B$ tordues par un caractère de $B^{\times}$.

Distributions $(G, \kappa)$-invariantes sur $A$. Soit $\kappa \in \epsilon\left(\left(F^{\times}\right)^{n} \backslash F^{\times}\right)$. Pour $f \in C_{\mathrm{c}}^{\infty}(A)$ et $g \in G$, on note ${ }^{g, \kappa} f \in C_{\mathrm{c}}^{\infty}(A)$ la fonction définie par

$$
{ }^{g, \kappa} f(y)=\kappa \circ \operatorname{det}^{\prime} g f\left(g^{-1} y g\right) .
$$

Une distribution $T \in \mathscr{D}(A)$ est dite $(G, \kappa)$-invariante $\operatorname{si}\left\langle{ }^{g, \kappa} f, T\right\rangle=\langle f, T\rangle$ pour tous $f \in C_{\mathrm{c}}^{\infty}(A)$ et $g \in G$. Soit $J_{\kappa}(A) \subset \mathscr{D}(A)$ le sous-espace formé des distributions $(G, \kappa)$-invariantes. On a clairement $J_{\kappa}(A) \subset J_{H_{\kappa}}(A)$ avec $H_{\kappa}=\operatorname{ker}\left(\kappa \circ \operatorname{det}^{\prime}\right)$.

Pour chaque $\alpha \in \Pi_{n}$, choisissons une mesure de Haar $d g_{n_{\alpha}}$ sur $G_{n_{\alpha}}$. Pour $\alpha \in \Pi_{n}$ et $\kappa \in \epsilon\left(\Delta_{\alpha} \backslash F^{\times}\right)$, on note $\Theta_{\alpha}^{\kappa}=\Theta_{\alpha}^{\kappa}\left(\cdot, d g_{n_{\alpha}}\right) \in J_{\kappa}(A)$ la distribution définie par

$$
\left\langle f, \Theta_{\alpha}^{\kappa}\right\rangle=\int_{G_{n_{\alpha}} \backslash G} \kappa \circ \operatorname{det}^{\prime} g f\left(g^{-1} n_{\alpha} g\right) \frac{d g}{d g_{n_{\alpha}}} \quad\left(f \in C_{\mathrm{c}}^{\infty}(A)\right) ;
$$

d'après [Lemaire 2004, 1.10/2], l'intégrale est absolument convergente. Soient $\alpha \in$ $\Pi_{n}$ et $\kappa \in \epsilon\left(\Delta_{\alpha} \backslash F^{\times}\right)$. Puisque le groupe $\Delta_{\alpha} \backslash F^{\times}$est compact, le caractère $\kappa$ se factorise à travers un quotient fini $U \backslash F^{\times}$de $\Delta_{\alpha} \backslash F^{\times}$; i.e., il existe un sous-groupe ouvert $U \subset F^{\times}$contenant $\Delta_{\alpha}$ tel que $\left.\kappa\right|_{U}=1$. Soit $H$ un sous-groupe ouvert distingué de $G$ tel que $\Delta_{H} \subset U$ (un tel groupe existe). Notons $d h_{n_{\alpha}}$ la mesure de Haar sur $H_{n_{\alpha}}$ définie par

$$
d h_{n_{\alpha}}=\left.d g_{n_{\alpha}}\right|_{H_{n_{\alpha}}},
$$

et $\Theta_{\alpha, \bar{x}} \in J_{H}(A)\left(\bar{x} \in \Delta_{H} \Delta_{\alpha} \backslash F^{\times}\right)$la distribution définie grâce aux mesures $d h$ et $d h_{n_{\alpha}}$ comme en 1.5. Pour chaque $\bar{x} \in \Delta_{H} \Delta_{\alpha} \backslash F^{\times}$, choisissons un élément $x \in P_{\alpha}$ 
tel que $\operatorname{det}^{\prime}(x)\left(\bmod \Delta_{H} \Delta_{\alpha}\right)=\bar{x}$. Pour $f \in C_{\mathrm{c}}^{\infty}(A)$, on a

$$
\begin{aligned}
\left\langle f, \Theta_{\alpha}^{\kappa}\right\rangle & =\sum_{\bar{x} \in \Delta_{H} \Delta_{\alpha} \backslash F^{\times}} \kappa \circ \operatorname{det}^{\prime}(x) \int_{H_{n_{\alpha}} \backslash H} \operatorname{Ad}^{*} x(f)\left(h^{-1} n_{\alpha} h\right) \frac{d h}{d h_{n_{\alpha}}} \\
& =\sum_{\bar{x} \in \Delta_{H} \Delta_{\alpha} \backslash F^{\times}} \kappa(\bar{x})\left\langle f, \Theta_{\alpha, \bar{x}}\right\rangle ;
\end{aligned}
$$

c'est-à-dire

$$
\Theta_{\alpha}^{\kappa}=\sum_{\bar{x} \in \Delta_{H} \Delta_{\alpha} \backslash F^{\times}} \kappa(\bar{x}) \Theta_{\alpha, \bar{x}}
$$

Réciproquement, pour $\bar{x} \in \Delta_{H} \Delta_{\alpha} \backslash F^{\times}$, on a

$$
\Theta_{\alpha, \bar{x}}=\left(F^{\times}: \Delta_{H} \Delta_{\alpha}\right)^{-1} \sum_{\kappa \in \epsilon\left(\Delta_{H} \Delta_{\alpha} \backslash F^{\times}\right)} \kappa(\bar{x})^{-1} \Theta_{\alpha}^{\kappa} .
$$

Soit $\Pi_{n}^{\prime}$ l'ensemble des couples $(\alpha, \kappa)$ avec $\alpha \in \Pi_{n}$ et $\kappa \in \epsilon\left(\Delta_{\alpha} \backslash F^{\times}\right)$. Puisque $\left(F^{\times}\right)^{n}=\Delta_{(n)} \subset \Delta_{\alpha}\left(\alpha \in \Pi_{n}\right)$, le sous-groupe $\epsilon\left(\left(F^{\times}\right)^{n} \backslash F^{\times}\right) \subset \epsilon\left(F^{\times}\right)$coïncide avec l'image de l'application $\Pi_{n}^{\prime} \rightarrow \epsilon\left(F^{\times}\right),(\alpha, \kappa) \mapsto \kappa$. Pour $r \in \mathbb{Z}_{>0}$ tel que $r$ divise $n$, notons $\varphi_{n / r, n}: \Pi_{n / r} \rightarrow \Pi_{n}$ l'application injective définie par

$$
\varphi(\beta)=\left(r \beta_{1} \geq \cdots \geq r \beta_{n / r} \geq 0=\cdots=0\right) \quad\left(\beta=\left(\beta_{1} \geq \cdots \geq \beta_{n / r}\right) \in \Pi_{n / r}\right) .
$$

Pour $\alpha \in \Pi_{n}$, notons $s(\alpha)$ le plus grand diviseur commun des $\alpha_{i}(i=1, \ldots, n)$. On a clairement $\Delta_{\alpha}=\left(F^{\times}\right)^{s(\alpha)}\left(\alpha \in \Pi_{n}\right)$. Par conséquent pour $\alpha \in \Pi_{n}$ et $\kappa \in$ $\epsilon\left(\left(F^{\times}\right)^{n} \backslash F^{\times}\right)$, on a $(\alpha, \kappa) \in \Pi_{n}^{\prime}$ si et seulement si $r(\kappa)$ divise $s(\alpha)$; où $r(\kappa)$ désigne l'ordre de $\kappa$. On en déduit que pour $\kappa \in \epsilon\left(\left(F^{\times}\right)^{n} \backslash F^{\times}\right)$, la fibre au-dessus de $\kappa$ pour l'application $\Pi_{n}^{\prime} \rightarrow \epsilon\left(F^{\times}\right),(\alpha, \kappa) \mapsto \kappa$ s'identifie canoniquement à $\Pi_{n / r(\kappa)}$ via l'application $\varphi_{n / r(\kappa), n}$.

Lemme 6.6. Les distributions $\Theta_{\alpha}^{\kappa}\left((\alpha, \kappa) \in \Pi_{n}^{\prime}\right)$ sont invariantes par un sousgroupe ouvert distingué de $G$ d'indice fini, linéairement indépendantes, et forment une base des distributions sur A à support dans $\mathfrak{N}$ invariantes par un sous-groupe ouvert distingué de $G$.

Démonstration. Pour $(\alpha, \kappa) \in \Pi_{n}^{\prime}$, la distribution $\Theta_{\alpha}^{\kappa}$ est $H_{\kappa}$-invariante; et $H_{\kappa}$ est un sous-groupe ouvert distingué de $G$ d'indice fini.

Soit $\left\{a_{\alpha, \kappa}:(\alpha, \kappa) \in \Pi_{n}^{\prime}\right\}$ une famille de nombres complexes presque tous nuls, telle que $\sum_{(\alpha, \kappa) \in \Pi_{n}^{\prime}} a_{\alpha, \kappa} \Theta_{\alpha}^{\kappa}=0$. Soit $H$ un sous-groupe ouvert distingué de $G$ tel que $\Delta_{H} \subset \operatorname{ker} \kappa$ pour tout $(\alpha, \kappa) \in \Pi_{n}^{\prime}$ tel que $a_{\alpha, \kappa} \neq 0$. Pour $(\alpha, \bar{x}) \in \Pi_{H, n}$, on 
définit comme plus haut une distribution $\Theta_{\alpha, \bar{x}} \in J_{H}(A)$. D'après (6.4), on a

$$
\begin{aligned}
0=\sum_{(\alpha, \kappa) \in \Pi_{n}^{\prime}} a_{\alpha, \kappa} \Theta_{\alpha}^{\kappa} & =\sum_{(\alpha, \kappa) \in \Pi_{n}^{\prime}} a_{\alpha, \kappa}\left(\sum_{\bar{x} \in \Delta_{H} \Delta_{\alpha} \backslash F^{\times}} \kappa(\bar{x}) \Theta_{\alpha, \bar{x}}\right) \\
& =\sum_{(\alpha, \bar{x}) \in \Pi_{H, n}}\left(\sum_{\kappa \in \epsilon\left(\Delta_{H} \Delta_{\alpha} \backslash F^{\times}\right)} a_{\alpha, \kappa} \kappa(\bar{x})\right) \Theta_{\alpha, \bar{x}} .
\end{aligned}
$$

Les distributions $\Theta_{\alpha, \bar{x}}\left((\alpha, \bar{x}) \in \Pi_{H, n}\right)$ forment une base de $J_{H}(\mathfrak{N})$. Par conséquent, pour $(\alpha, \bar{x}) \in \Pi_{H, n}$, on a

$$
\sum_{\kappa \in \epsilon\left(\Delta_{H} \Delta_{\alpha} \backslash F^{\times}\right)} a_{\alpha, \kappa} \kappa(\bar{x})=0 .
$$

En d'autres termes, pour $\alpha \in \Pi_{n}$, on a $\sum_{\kappa \in \epsilon\left(\Delta_{H} \Delta_{\alpha} \backslash F^{\times}\right)} a_{\alpha, \kappa} \kappa=0$; ce qui implique que $a_{\alpha, \kappa}=0$ pour tout $(\alpha, \kappa) \in \Pi_{n}^{\prime}$. Les distributions $\Theta_{\alpha}^{\kappa}\left((\alpha, \kappa) \in \Pi_{n}^{\prime}\right)$ sont donc linéairement indépendantes.

Enfin d'après (6.5), pour tout sous-groupe ouvert distingué $H \subset G$, les distributions $\Theta_{\alpha}^{\kappa}\left(\alpha \in \Pi_{n}, \kappa \in \epsilon\left(\Delta_{H} \Delta_{\alpha} \backslash F^{\times}\right)\right)$engendrent l'espace $J_{H}(\mathfrak{N}) ;$ d'où la dernière assertion du lemme.

On en déduit :

Proposition 6.7. Soit $\pi^{\prime} \in \epsilon\left(G^{\prime}\right)$. Il existe un entier $m>0$ et des constantes $c_{\alpha}^{\kappa}\left(\pi^{\prime}\right)$ $\left((\alpha, \kappa) \in \Pi_{n}^{\prime}\right)$ presque toutes nulles, tels que

$$
\Theta_{\pi^{\prime}}(1+u)=\sum_{(\alpha, \kappa) \in \Pi_{n}^{\prime}} c_{\alpha}^{\kappa}\left(\pi^{\prime}\right)\left(\Theta_{\alpha}^{\kappa}\right)^{\vee}(u)
$$

pour tout $u \in \mathfrak{A}^{m} \cap A^{\prime}$ tel que $1+u \in G^{\prime}$.

On peut de la même manière remplacer dans la formule (6.2) les distributions $\Theta_{\natural, n}^{\vee}$ par des combinaisons linéaires des transformées de Fourier des $B^{\times}$-intégrales orbitales nilpotentes sur $B$ tordues par un caractère de $B^{\times}$(on laisse au lecteur le soin d'écrire la formule).

Remarque 6.8. Soit $H \subset G$ un sous-groupe ouvert distingué. On sait que les distributions $\Theta_{\alpha, \bar{x}}\left((\alpha, \bar{x}) \in \Pi_{H, n}\right)$ forment une base du $\mathbb{C}$-espace vectoriel $J_{H}(\mathfrak{N})$. Par conséquent, pour $v \in \frac{1}{d} \mathbb{Z}$, les formes linéaires $j^{v}\left(\Theta_{\alpha, \bar{x}}\right)\left((\alpha, \bar{x}) \in \Pi_{H, n}\right)$ sur $C_{\mathrm{c}}\left(A / \mathfrak{A}^{v}\right)$, engendrent le $\mathbb{C}$-espace vectoriel $j^{v}\left(J_{H}(\mathfrak{N})\right)$. Soit $v \in \frac{1}{d} \mathbb{Z}$, et soit $\left\{f_{k}\right\}_{k=0}^{s} \subset C_{\mathrm{c}}^{\infty}(A)$ un jeu de fonctions vérifiant la condition (2) du lemme 2.20. Il existe un $t \in F^{\times}$tel que pour $i=0, \ldots, s$, on a $\left(f_{i}\right)_{t} \in C_{\mathrm{c}}\left(A / \mathfrak{A}^{v}\right)$ (voir la démonstration de la proposition 2.23 pour la définition de $\left.\left(f_{i}\right)_{t}\right)$. D'après la formule d'homogénéité pour $\Theta_{\alpha, \bar{x}}$ donnée au début de la démonstration du lemme 2.26, pour $i, j \in\{0, \ldots, s\}$, on a $\left\langle\left(f_{i}\right)_{t}, \Theta_{j}\right\rangle=|t|^{\operatorname{dim}\left(\mathscr{C}_{i}\right) / 2} \delta_{i, j}$. Par suite, les formes linéaires $j^{v}\left(\Theta_{\alpha, \bar{x}}\right)\left((\alpha, \bar{x}) \in \Pi_{H, n}\right)$ sur $C_{\mathrm{c}}\left(A / \mathfrak{A}^{v}\right)$ sont linéairement indépendantes. 
Par dualité, on en déduit que les constantes $c_{\alpha, \bar{x}}(T)$ apparaissant dans la proposition 1.6 sont uniques. De même (cf. la démonstration du lemme 6.6), pour $v \in \frac{1}{d} \mathbb{Z}$, les formes linéaires $j^{v}\left(\Theta_{\alpha}^{\kappa}\right)\left((\alpha, \kappa) \in \Pi_{n}^{\prime}\right)$ sur $C_{c}\left(A / \mathfrak{A}^{v}\right)$, sont linéairement indépendantes; par conséquent les constantes $c_{\alpha}^{\kappa}\left(\pi^{\prime}\right)$ apparaissant dans la proposition 6.7 sont uniques.

Soit $\left(\pi^{\prime}, W\right)$ une représentation complexe lisse irréductible de $G^{\prime}$. D'après la remarque 6.8 , le groupe

$$
H_{\pi^{\prime}}=\bigcap_{(\alpha, \kappa)} H_{\kappa}
$$

où $(\alpha, \kappa)$ parcourt les éléments de $\Pi_{n}^{\prime}$ tels que $c_{\alpha}^{\kappa}\left(\pi^{\prime}\right) \neq 0$, est bien défini. Et si $H$ est un sous-groupe ouvert d'indice fini de $G$, contenant ${ }_{\varpi} G^{\prime}$ et tel que $\pi^{\prime}$ s'étende en une représentation lisse $(\pi, W)$ de $H$, alors on a nécessairement l'inclusion $H \subset H_{\pi^{\prime}}$.

\section{Application : intégrabilité locale des caractères tordus des représentations $\kappa$-stables de $\operatorname{GL}_{n}(D)$.}

Soit $\kappa \in \epsilon\left(\left(F^{\times}\right)^{n} \backslash F^{\times}\right)$. Une distribution $T \in \mathscr{D}(G)$ est dite $(G, \kappa)$-invariante si $\left\langle{ }^{g, \kappa} f, T\right\rangle=\langle f, T\rangle$ pour tous $f \in C_{\mathrm{c}}^{\infty}(G)$ et $g \in G$. Soit $J_{\kappa}(G) \subset \mathscr{D}(G)$ le sousespace formé des distributions $(G, \kappa)$-invariantes. On a clairement $J_{\kappa}(G) \subset J_{H_{\kappa}}(G)$ avec (rappel) $H_{\kappa}=\operatorname{ker}\left(\kappa \circ \operatorname{det}^{\prime}\right)$.

On rappelle qu'une représentation complexe lisse $\pi$ de $G$ est dite $\kappa$-stable si $\kappa \pi \simeq \pi$ avec $\kappa \pi=\left(\kappa \circ \operatorname{det}^{\prime}\right) \otimes \pi$. Soit $\tilde{\epsilon}_{\kappa}(G)$ un système de représentants des classes d'équivalence de représentations complexes lisses irréductibles $\kappa$-stables de $G$. Pour $\pi \in \tilde{\epsilon}_{\kappa}(G)$, on choisit un automorphisme non nul $A_{\pi}^{\kappa}$ de l'espace $V_{\pi}$ de $\pi$ tel que $\pi(g) \circ A_{\pi}^{\kappa}=A_{\pi}^{\kappa} \circ(\kappa \pi)(g)(g \in G)$, et l'on note $\Theta_{\pi}^{\kappa}=\operatorname{trace}\left(\pi d g \circ A_{\pi}^{\kappa}\right) \in J_{\kappa}(G)$ la distribution définie $\operatorname{par}\left\langle f, \Theta_{\pi, \kappa}\right\rangle=\operatorname{trace}\left(\pi(f d g) \circ A_{\pi}^{\kappa}\right)$. D'après le lemme de Schur, $A_{\pi}^{\kappa}$ est unique à multiplication près par une constante complexe non nulle. Une représentation complexe lisse $\rho$ de $H=H_{\kappa}$ est dite régulière si pour tout $g \in G \backslash H$, on a $\rho^{g} \not \subset \rho$ avec $\rho^{g}(h)=\rho\left(g h g^{-1}\right)(h \in H)$. Notons $\epsilon^{\prime}(H) \subset \epsilon(H)$ le sous-ensemble formé des classes de représentations régulières.

D'après [Kazhdan 1984, section 2, lemma 2.1], l'application $\rho \mapsto \operatorname{ind}_{H}^{G} \rho$ induit une bijection entre

- l'ensemble des $G$-orbites dans $\epsilon^{\prime}(H)$,

- l'ensemble des classes d'équivalence de représentations complexes lisses irréductibles $\kappa$-stables de $G$.

De plus, si $\pi \in \tilde{\epsilon}_{\kappa}(G)$ et si $\rho$ est une représentation complexe lisse régulière de $H$ telle que $\pi \simeq \operatorname{ind}_{H}^{G} \rho$, alors il existe une constante complexe $c=c_{\pi}(\rho) \neq 0$ (dépendant du choix de l'opérateur d'entrelacement $A_{\pi}^{\kappa}$ ) telle que pour toute fonction 
$f \in C_{\mathrm{c}}^{\infty}(G)$, on a la formule

$$
\left\langle f, \Theta_{\pi}^{\kappa}\right\rangle=c \sum_{g \in H \backslash G} \kappa \circ \operatorname{det}^{\prime}(g)\left\langle\left. f\right|_{H}, \Theta_{\rho^{g}}\right\rangle ;
$$

ici, les caractères $\Theta_{\rho^{g}}$ sont définis par $\Theta_{\tau}=\operatorname{trace}(\tau d h)(\tau \in \epsilon(H))$ avec $d h=\left.d g\right|_{H}$. Notons que la constante $c_{\pi}(\rho)$ ne dépend pas vraiment de $\rho$ mais seulement de la classe d'équivalence de $\rho$, et que pour $g \in G$, on a $c_{\pi}\left(\rho^{g}\right)=\kappa(g) c_{\pi}(\rho)$.

On en obtient :

Proposition 7.2. Soit $\pi \in \tilde{\epsilon}_{\kappa}(G)$. La distribution $\Theta_{\pi}^{\kappa}$ est localement intégrable sur $G$, localement constante sur $G_{\mathrm{r}}$. De plus, on a $\operatorname{Supp}\left(\Theta_{\pi}^{\kappa}\right) \subset H_{\kappa}$.

Démonstration. Soit $\rho$ une représentation complexe lisse régulière de $H$ telle que $\pi \simeq \operatorname{ind}_{H}^{G} \rho$. D'après (7.1) et le théorème 3.10 , il existe une constante complexe $c=c_{\pi}(\rho) \neq 0$ telle que pour toute fonction $f \in C_{\mathrm{c}}^{\infty}(G)$, on a

$$
\left\langle f, \Theta_{\pi}^{\kappa}\right\rangle=c \int_{H} f(h)\left(\sum_{g \in H \backslash G} \kappa \circ \operatorname{det}^{\prime}(g) \Theta_{\rho^{g}}(h)\right) d h
$$

(intégrale absolument convergente). On a donc $\operatorname{Supp}\left(\Theta_{\pi}^{\kappa}\right) \subset H$; et puisque les fonctions $\left.\Theta_{\rho^{g}}\right|_{H_{\mathrm{r}}}(g \in H \backslash G)$ sont localement constantes, la fonction $\left.\Theta_{\pi}^{\kappa}\right|_{G_{\mathrm{r}}}$ est localement constante.

Posons $r=r(\kappa), n^{\prime}=n / r(\kappa)$ et $\varphi=\varphi_{n^{\prime}, n}: \Pi_{n^{\prime}} \rightarrow \Pi_{n}$.

Corollaire 7.3. Il existe un entier $m>0$ et des constantes $c_{\beta}(\pi)\left(\beta \in \Pi_{n^{\prime}}\right)$ tels que

$$
\Theta_{\pi}^{\kappa}(1+u)=\sum_{\beta \in \Pi_{n^{\prime}}} c_{\beta}(\pi)\left(\Theta_{\varphi(\beta)}^{\kappa}\right)^{\vee}(u)
$$

pour presque tout $u \in \mathfrak{A}^{m} \cap A$ (resp. pour tout $\left.u \in \mathfrak{A}^{m} \cap A^{\prime}\right)$.

Démonstration. D'après la démonstration de 7.2, il existe une constante $c>0$ telle que pour presque tout $h \in H$ (resp. pour tout $h \in H_{\mathrm{r}}$ ), on a

$$
\Theta_{\pi}^{\kappa}(h)=c \sum_{g \in H \backslash G} \kappa \circ \operatorname{det}^{\prime}(g) \Theta_{\rho^{g}}(h) .
$$

Et d'après 3.8, il existe un entier $m_{1}>0$ tel que $K^{m_{1}} \subset H$ et des constantes $c_{\alpha, \bar{x}}(\rho)$ $\left((\alpha, \bar{x}) \in \Pi_{H, n}\right)$, tels que

$$
\Theta_{\rho}(1+u)=\sum_{(\alpha, \bar{x}) \in \Pi_{H, n}} c_{\alpha, \bar{x}}(\rho) \Theta_{\alpha, \bar{x}}^{\vee}(u)
$$

pour tout $u \in \mathfrak{A}^{m_{1}} \cap A^{\prime}$. Soient $g_{1}, \ldots, g_{s} \in G$ tels que $G=\coprod_{i=1}^{s} H g_{i}$ (union disjointe), et soit un entier $m \geq m_{1}$ tel que $\mathfrak{A}^{m} \subset \bigcap_{i=1}^{s} g_{i} \mathfrak{A}^{m_{1}} g_{i}^{-1}$. Ainsi, pour 
$i=1, \ldots, s$, on a

$$
\Theta_{\rho^{g_{i}}}(1+u)=\Theta_{\rho}\left(1+g_{i}^{-1} u g_{i}\right)=\sum_{(\alpha, \bar{x}) \in \Pi_{H, n}} c_{\alpha, \bar{x}}(\rho) \Theta_{\alpha, \bar{x}}^{\vee}\left(g_{i}^{-1} u g_{i}\right)
$$

pour tout $u \in \mathfrak{A}^{m} \cap A^{\prime}$. D'où l'on déduit que

$$
\Theta_{\pi}^{\kappa}(1+u)=\sum_{(\alpha, \bar{x}) \in \Pi_{H, n}} c c_{\alpha, \bar{x}}(\rho)\left(\sum_{i=1}^{s} \kappa \circ \operatorname{det}^{\prime}\left(g_{i}\right) \Theta_{\alpha, \bar{x}}^{\vee}\left(g_{i}^{-1} u g_{i}\right)\right)
$$

pour tout $u \in \mathfrak{A}^{m} \cap A^{\prime}$. Pour $\left(\alpha, \kappa^{\prime}\right) \in \Pi_{n}^{\prime}$, on a $\Theta_{\alpha}^{\kappa^{\prime}} \in J_{\kappa^{\prime}}(A)$; par conséquent $\left(\Theta_{\alpha}^{\kappa^{\prime}}\right)^{\vee} \in J_{\kappa^{\prime}}(A)$ et

$$
\left(\Theta_{\alpha}^{\kappa^{\prime}}\right)^{\vee}\left(g^{-1} u g\right)=\kappa^{\prime} \circ \operatorname{det}^{\prime}(g)\left(\Theta_{\alpha}^{\kappa^{\prime}}\right)^{\vee}(u) \quad\left(g \in G, u \in A^{\prime}\right) .
$$

Posons $c_{H, \alpha}=\left(F^{\times}: \Delta_{H} \Delta_{\alpha}\right)^{-1}\left(\alpha \in \Pi_{n}\right)$. D'après (6.5), pour $(\alpha, \bar{x}) \in \Pi_{H, n}$ et $i=1, \ldots, s$, on a

$$
\Theta_{\alpha, \bar{x}}^{\vee}\left(g_{i}^{-1} u g_{i}\right)=c_{H, \alpha} \sum_{\kappa^{\prime}} \kappa^{\prime}(\bar{x})^{-1} \kappa^{\prime} \circ \operatorname{det}^{\prime}\left(g_{i}\right)\left(\Theta_{\alpha}^{\kappa^{\prime}}\right)^{\vee}(u) \quad\left(u \in A^{\prime}\right)
$$

où $\kappa^{\prime}$ parcourt les éléments de $\epsilon\left(\Delta_{H} \Delta_{\alpha} \backslash F^{\times}\right)$. On en déduit que pour $(\alpha, \bar{x}) \in \Pi_{H, n}$ et $u \in A^{\prime}$, on a

$$
\sum_{i=1}^{s} \kappa \circ \operatorname{det}^{\prime}\left(g_{i}\right) \Theta_{\alpha, \bar{x}}^{\vee}\left(g_{i}^{-1} u g_{i}\right)= \begin{cases}0 & \text { si }\left.\kappa\right|_{\Delta_{\alpha}} \neq 1, \\ s c_{H, \alpha} \kappa(\bar{x})\left(\Theta_{\alpha}^{\kappa}\right)^{\vee}(u) & \text { sinon. }\end{cases}
$$

Mais pour $\alpha \in \Pi_{n}$, on a $\left.\kappa\right|_{\Delta_{\alpha}}=1$ si et seulement si $r(\kappa)$ divise $s(\alpha)$; i.e., si et seulement si $\alpha \in \varphi\left(\Pi_{n^{\prime}}\right)$. Par ailleurs, pour $\beta \in \Pi_{n^{\prime}}$, on a $\Delta_{\varphi(\beta)} \subset \Delta_{H}$, d'où $s c_{H, \varphi(\beta)}=1$. En définitive, on obtient que

$$
\Theta_{\pi}^{\kappa}(1+u)=\sum_{\beta \in \Pi_{n^{\prime}}}\left(\sum_{\bar{x} \in \Delta_{H} \backslash F^{\times}} c c_{\varphi(\beta), \bar{x}}(\rho) \kappa(\bar{x})\right)\left(\Theta_{\varphi(\beta)}^{\kappa}\right)^{\vee}(u)
$$

pour tout $u \in \mathfrak{A}^{m} \cap A^{\prime}$. D'où le corollaire.

\section{Appendice : à propos des distributions $\left(\Theta_{\alpha}^{\kappa}\right)^{\vee}$}

Soit $(\alpha, \kappa) \in \Pi_{n}^{\prime}$. Si $\alpha=(1, \ldots, 1)$, alors $\kappa=1$ et $\left(\Theta_{\alpha}^{\kappa}\right)^{\vee}$ est une mesure de Haar sur $A$. On suppose donc que $\alpha \neq(1, \ldots, 1)$. Posons $H=H_{\kappa}$; on a donc $\Delta_{\alpha} \subset \Delta_{H}$. Comme dans la section 1 , la $P_{\alpha}$-orbite $O_{\alpha}^{\bullet}$ se décompose en $\mathrm{O}_{\alpha}^{\bullet}=\bigsqcup_{\bar{x}} \mathrm{O}_{\alpha, \bar{x}}^{\bullet}$ où $\bar{x}$ parcourt les éléments de $\Delta_{H} \backslash F^{\times}$. Pour $u \in \mathfrak{u}_{\alpha}$, on note $\varphi(u) \in \Delta_{H} \backslash F^{\times}$l'élément défini par

$$
\varphi(u)= \begin{cases}1 & \text { si } u \in \mathfrak{U}_{\alpha} \backslash \mathbb{O}_{\alpha}^{\bullet}, \\ \bar{x} & \text { si } u \in \mathcal{O}_{\alpha, \bar{x}}^{\bullet}\end{cases}
$$


Pour $f \in C_{\mathrm{c}}^{\infty}(A)$, notons $\tilde{f} \in C_{\mathrm{c}}^{\infty}(A)$ la fonction $g \mapsto \int_{K_{H}} f\left(k^{-1} g k\right) d k$ où $d k$ désigne la mesure de Haar normalisée sur $K_{H}$. D'après (6.4) et 1.5, il existe une constante $c>0$ telle que pour toute fonction $f \in C_{\mathrm{c}}^{\infty}(A)$, on a

$$
\begin{aligned}
\left\langle f, \Theta_{\alpha}^{\kappa}\right\rangle & =c \sum_{\bar{x} \in \Delta_{H} \backslash F^{\times}} \kappa(\bar{x}) \iint_{K_{H} \times \overline{\bar{O}_{\alpha, \bar{x}}}} f\left(k^{-1} u k\right) d k d u \\
& =c \int_{\mathfrak{u}_{\alpha}} \kappa \circ \varphi(u) \tilde{f}(u) d u .
\end{aligned}
$$

Par construction, on a

$$
\kappa \circ \varphi\left(p^{-1} u p\right)=\kappa \circ \operatorname{det}^{\prime}(p) \kappa \circ \varphi(u) \quad\left(u \in \mathfrak{u}_{\alpha}, p \in P_{\alpha}\right) .
$$

Posons $n^{\prime}=n / r(\kappa)$, et soit $\beta=\varphi_{n^{\prime}, n}^{-1}(\alpha) \in \Pi_{n^{\prime}}$. La partition duale de $\alpha$,

$$
\hat{\alpha}=\left(\hat{\alpha}_{1} \geq \cdots \geq \hat{\alpha}_{n}\right) \in \Pi_{n},
$$

est donnée par

$$
\hat{\alpha}=\left(\hat{\beta}_{1}=\cdots=\hat{\beta}_{1} \geq \hat{\beta}_{2}=\cdots=\hat{\beta}_{2} \geq \cdots \geq \hat{\beta}_{n^{\prime}}=\cdots=\hat{\beta}_{n^{\prime}}\right)
$$

où chaque $\hat{\beta}_{i}$ apparaît $r(\kappa)$ fois. On a $\hat{\alpha}_{i}=\operatorname{dim}_{D}\left(V_{\alpha}^{i}\right)-\operatorname{dim}_{D}\left(V_{\alpha}^{i-1}\right)\left(i=1, \ldots, r_{\alpha}\right)$; en particulier, on a $\hat{\alpha}_{r_{\alpha}+1}=\cdots=\hat{\alpha}_{n}=0$. Pour $i=1, \ldots, r_{\alpha}$, notons $W_{\alpha}^{i}$ l'unique sous- $D$-espace vectoriel de $V_{\alpha}^{i}$ de la forme $W_{\alpha}^{i}=\bigoplus_{j \in I(\alpha, i)} W^{j}$ pour un sousensemble fini $I(\alpha, i) \subset\{1, \ldots, n\}$, tel que $V_{\alpha}^{i}=V_{\alpha}^{i-1} \oplus W_{\alpha}^{i}$ (pour la définition des $W^{j}$, voir le paragraphe "Descente parabolique", page 83); on a $\operatorname{donc} \operatorname{dim}_{D}\left(W_{\alpha}^{i}\right)=$ $\hat{\beta}_{i}$. Pour $i, j \in\left\{1, \ldots, r_{\alpha}\right\}, A_{\alpha}^{i, j}=\operatorname{End}_{D}\left(W_{\alpha}^{j}, W_{\alpha}^{i}\right)$ s'identifie canoniquement à un sous- $F$-espace vectoriel de $A$. On a les décompositions

$$
\mathfrak{m}_{\alpha}=\bigoplus_{i=1}^{r_{\alpha}} A_{\alpha}^{i, i}, \quad \mathfrak{u}_{\alpha}=\bigoplus_{i<j} A_{\alpha}^{i, j}, \quad \bar{u}_{\alpha}=\bigoplus_{i>j} A_{\alpha}^{i, j} .
$$

Écrivons $n_{\alpha}=\bigoplus_{i<j} n_{\alpha}^{i, j}$ avec $n_{\alpha}^{i, j} \in \mathfrak{u}_{\alpha}^{i, j}$. Si $i \in\left\{1, \ldots, r_{\alpha}-1\right\}$ est un indice tel que $\hat{\alpha}_{i}=\hat{\alpha}_{i+1}$, on a (voir [Lemaire 2004, 1.4/5]) $n_{\alpha}^{i, i+1} \in \operatorname{Isom}_{D}\left(W_{\alpha}^{i+1}, W_{\alpha}^{i}\right)$; et pour $x \in A_{\alpha}^{i, i+1}$, on note $\operatorname{det}^{\prime}(x)$ le déterminant réduit de $x$ défini via $n_{\alpha}^{i, i+1}$ : $\operatorname{det}^{\prime}(x)=\operatorname{det}^{\prime}\left(x \circ\left(n_{\alpha}^{i, i+1}\right)^{-1}\right)$. Comme dans [Hales 1993, 3.6], on définit comme suit une fonction $b_{\kappa}: \mathbb{O}_{\alpha}^{\bullet}\left(=\mathbb{O}_{P_{\alpha}}\left(n_{\alpha}\right)\right) \rightarrow \mathbb{C}:$ pour $u=\bigoplus_{i<j} u_{i, j} \in \mathbb{O}_{\alpha}^{\bullet}$ avec $u_{i, j} \in A_{\alpha}^{i, j}$, on pose

$$
b_{\kappa}(u)=\prod_{i=1}^{r_{\alpha}-1} \kappa^{-i}\left(\operatorname{det}^{\prime}\left(u_{i, i+1}\right)\right)
$$

avec (convention d'écriture) $\kappa^{-i}\left(\operatorname{det}^{\prime}\left(u_{i, i+1}\right)\right)=1$ si $\hat{\alpha}_{i} \neq \hat{\alpha}_{i+1}$; notons que si $\hat{\alpha}_{i} \neq \hat{\alpha}_{i+1}$, alors $r(\kappa)$ divise $i$ et $\kappa^{-i}=1$. Comme dans [Hales 1993, 3.6], on 
montre que

$$
b_{\kappa}\left(p^{-1} u p\right)=\kappa \circ \operatorname{det}^{\prime}(p) b_{\kappa}(u) \quad\left(u \in \mathfrak{u}_{\alpha}, p \in P_{\alpha}\right) .
$$

Puisque $\mathcal{O}_{\alpha}^{\bullet}=\mathscr{O}_{P_{\alpha}}\left(n_{\alpha}\right)$ est dense dans $\mathfrak{u}_{\alpha}$, d'après (A.1) et (A.2), il existe une constante complexe $c^{\prime} \neq 0$ telle que $\kappa \circ \varphi(u)=c^{\prime} b_{\kappa}(u)$ pour presque tout $u \in \mathfrak{u}_{\alpha}$. Comme $\kappa \circ \varphi\left(n_{\alpha}\right)=1=b_{\kappa}\left(n_{\alpha}\right)$, on a $c^{\prime}=1$. D'où la formule

$$
\left\langle f, \Theta_{\alpha}^{\kappa}\right\rangle=c \int_{\mathfrak{u}_{\alpha}} b_{\kappa}(u) \tilde{f}(u) d u \quad\left(f \in C_{\mathrm{c}}^{\infty}(A)\right) .
$$

D'après la proposition 2.7 , on a donc

$$
\left(\Theta_{\alpha}^{\kappa}\right)^{\vee}(y)=c \int_{\mathfrak{u}_{\alpha}} b_{\kappa}(u) \bar{\Psi}_{A, y}^{K_{H}}(u) d u \quad\left(y \in A^{\prime}\right) .
$$

Notons que dans le cas très particulier où $D=F$ avec $\operatorname{car}(F)=0$, et $n$ est un nombre premier distinct de la caractéristique résiduelle de $F$, Assem [1994] a calculé explicitement cette fonction $\left.\left(\Theta_{\alpha}^{\kappa}\right)^{\vee}\right|_{A^{\prime}}$ (puisque $n$ est premier, on a nécessairement $\alpha=(n))$.

La distribution $\left(\Theta_{\alpha}^{\kappa}\right)^{\vee}$ est localement intégrable sur $A$ (proposition 2.1). On a donc

$$
\left\langle f,\left(\Theta_{\alpha}^{\kappa}\right)^{\vee}\right\rangle=\int_{A^{\prime}} f(g)\left(\Theta_{\alpha}^{\kappa}\right)^{\vee}(g) d_{A} g=\int_{A^{\prime}} \tilde{f}(g)\left(\Theta_{\alpha}^{\kappa}\right)^{\vee}(g) d_{A} g \quad\left(f \in C_{\mathrm{c}}^{\infty}(A)\right)
$$

(intégrales absolument convergentes). Puisque pour tout sous-groupe ouvert compact $\mathscr{K} \subset H$, la restriction $\left.\bar{\Psi}_{A, y}^{\mathscr{K}}\right|_{\mathfrak{u}_{\alpha}}$ est à support compact (corollaire 2.6), on en déduit que

$$
\left\langle f,\left(\Theta_{\alpha}^{\kappa}\right)^{\vee}\right\rangle=c \int_{A^{\prime}} \tilde{f}(y)\left(\int_{\mathfrak{u}_{\alpha}} b_{\kappa}(u) \bar{\Psi}_{A, y}^{\mathscr{K}}(u) d u\right) d_{A} y \quad\left(f \in C_{\mathrm{c}}^{\infty}(A)\right)
$$

pour tout sous-groupe ouvert $\mathscr{K} \subset K_{H}$.

Remarque A.6. Posons $\mathfrak{v}_{\alpha}=\bigoplus_{i=1}^{r_{\alpha}-1} A_{\alpha}^{i, i+1}$ et $\mathfrak{u}_{\alpha}^{1}=\bigoplus_{i<j-1}^{r_{\alpha}-1} A_{\alpha}^{i, j} \quad\left(=\left[\mathfrak{u}_{\alpha}, \mathfrak{u}_{\alpha}\right]\right)$; on a donc $\mathfrak{u}_{\alpha}=\mathfrak{v}_{\alpha} \oplus \mathfrak{u}_{\alpha}^{1}$. Soient $d v$ et $d u^{1}$ les mesures de Haar respectivement sur $\mathfrak{v}_{\alpha}$ et $\mathfrak{u}_{\alpha}^{1}$ normalisées par $\mathfrak{A}$. De même, posons $\overline{\mathfrak{v}}_{\alpha}=\bigoplus_{i=1}^{r_{\alpha}-1} A_{\alpha}^{i+1, i}$ et $\overline{\mathfrak{u}}_{\alpha}^{1}=\bigoplus_{i>j+1}^{r_{\alpha}-1} A_{\alpha}^{i, j}$; et soient $d \bar{v}$ et $d \bar{u}^{1}$ les mesures de Haar respectivement sur $\overline{\mathfrak{v}}_{\alpha}$ et $\overline{\mathfrak{u}}_{\alpha}^{1}$ normalisées par $\mathfrak{A}$. Enfin, posons $\mu_{\alpha}^{1}=\operatorname{vol}\left(\mathfrak{u}_{\alpha}^{1} \cap \mathfrak{A}^{1 / d}, d u^{1}\right)$.

Soit $f \in C_{\mathrm{c}}^{\infty}(A)$, et choisissons un $v \in \frac{1}{d} \mathbb{Z}$ tel que $f \in C_{\mathrm{c}}\left(A / \mathfrak{A}^{\nu}\right)$. Puisque $K_{H}$ normalise $\mathfrak{A}$, on a $\tilde{f} \in C_{\mathrm{c}}\left(A / \mathfrak{A}^{v}\right)$. Écrivons

$$
\tilde{f}=\sum_{i=1}^{r} \tilde{f}\left(y_{i}\right) \mathbf{1}_{y_{i}+\mathfrak{A}^{v}} .
$$


D'après (A.3), on a

$$
\begin{aligned}
\left\langle f,\left(\Theta_{\alpha}^{\kappa}\right)^{\vee}\right\rangle & =c \sum_{i=1}^{r} \tilde{f}\left(y_{i}\right) \int_{\mathfrak{u}_{\alpha}} b_{\kappa}(u) \overline{\Psi_{A}\left(u y_{i}\right)}\left(\int_{\mathfrak{A}^{v}} \overline{\Psi_{A}(u g)} d_{A} g\right) d u \\
& =c \operatorname{vol}\left(\mathfrak{A}^{v}, d_{A} g\right) \sum_{i=1}^{r} \tilde{f}\left(y_{i}\right) \int_{\mathfrak{u}_{\alpha} \cap \mathfrak{A}^{(1 / d)-v}} b_{\kappa}(u) \overline{\Psi_{A}\left(u y_{i}\right)} d u \\
& =c \int_{A} \tilde{f}(g)\left(\int_{\mathfrak{u}_{\alpha} \cap \mathfrak{A}^{(1 / d)-v}} b_{\kappa}(u) \overline{\Psi_{A}(u g)} \mathrm{du}\right) d_{A} g .
\end{aligned}
$$

Pour $\mu \in \frac{1}{d} \mathbb{Z}$, notons $\Phi_{\kappa}^{\mu}: A /\left(\mathfrak{p}_{\alpha}+\mathfrak{A}^{\nu}\right) \rightarrow \mathbb{C}$ et $\Psi_{\kappa}^{\mu}: A /\left(\overline{\mathfrak{u}}_{\alpha}^{1}+\mathfrak{p}_{\alpha}+\mathfrak{A}^{v}\right) \rightarrow \mathbb{C}$ les fonctions définies par

$$
\Phi_{\kappa}^{\mu}(g)=\int_{\mathfrak{u}_{\alpha} \cap \mathfrak{A}^{(1 / d)-\mu}} b_{\kappa}(u) \overline{\Psi_{A}(u g)} d u, \quad \Psi_{\kappa}^{\mu}(g)=\int_{\mathfrak{v}_{\alpha} \cap \mathfrak{A}^{(1 / d)-\mu}} b_{\kappa}(v) \overline{\Psi_{A}(v g)} d v .
$$

Pour $\mu \in \frac{1}{d} \mathbb{Z}$ et $\left(\bar{u}^{1}, \bar{v}\right) \in \overline{\mathfrak{u}}_{\alpha}^{1} \times \overline{\mathfrak{v}}_{\alpha}$, on a (calcul facile)

$$
\Phi_{\kappa}^{\mu}\left(\bar{u}^{1}+\bar{v}\right)=\operatorname{vol}\left(\mathfrak{u}^{1} \cap \mathfrak{A}^{(1 / d)-\mu}, d u^{1}\right) \mathbf{1}_{\overline{\mathfrak{u}}_{\alpha}^{1} \cap \mathfrak{A}^{\mu}}\left(\bar{u}^{1}\right) \Psi_{\kappa}^{\mu}(\bar{v})
$$

$\operatorname{avec} \operatorname{vol}\left(\mathfrak{u}_{\alpha}^{1} \cap \mathfrak{A}^{(1 / d)-\mu}, d u^{1}\right)=\mu_{\alpha}^{1} \operatorname{vol}\left(\mathfrak{u}_{\alpha}^{1} \cap \mathfrak{A}^{\mu}, d u^{1}\right)^{-1}$ et $\operatorname{vol}\left(\mathfrak{u}_{\alpha}^{1} \cap \mathfrak{A}^{\mu}, d u^{1}\right)=$ $\operatorname{vol}\left(\overline{\mathfrak{u}}_{\alpha}^{1} \cap \mathfrak{A}^{\mu}, d \bar{u}^{1}\right)$. Puisque $\left\langle f, \Theta_{\alpha, \kappa}^{\vee}\right\rangle=c \int_{\overline{\mathfrak{u}}_{\alpha} \times \mathfrak{p}_{\alpha}} \tilde{f}(\bar{u}+p) \Phi_{\kappa}^{v}(\bar{u}) d \bar{u} d p$, on en déduit que

$$
\left\langle f,\left(\Theta_{\alpha}^{\kappa}\right)^{\vee}\right\rangle=\mu_{\alpha}^{1} c \int_{\overline{\mathfrak{v}}_{\alpha} \times \mathfrak{p}_{\alpha}} \tilde{f}(\bar{v}+p) \Psi_{\kappa}^{v}(\bar{v}) d \bar{v} d p .
$$

L'égalité (A.7) est vraie pour tout couple $(f, v) \in C_{\mathrm{c}}^{\infty}(A) \times \frac{1}{d} \mathbb{Z}$ tel que $f \in$ $C_{\mathrm{c}}\left(A / \mathfrak{A}^{v}\right)$. En particulier, on a $\operatorname{Supp}\left(\left(\Theta_{\alpha}^{\kappa}\right)^{\vee}\right) \subset{ }^{K}\left(\overline{\mathfrak{v}}_{\alpha} \oplus \mathfrak{p}_{\alpha}\right)$. Pour $i=1, \ldots, r_{\alpha}-1$ tel que $\hat{\alpha}_{i}=\hat{\alpha}_{i+1}$, l'application $A_{\alpha}^{i, i+1} \times A_{\alpha}^{i+1,1} \rightarrow \mathbb{C},(v, \bar{v}) \mapsto \Psi_{A}(v \bar{v})$ met les groupes $A_{\alpha}^{i, i+1}$ et $A_{\alpha}^{i+1, i}$ en dualité. On peut donc à partir de (A.7) obtenir une formule intégrale analogue à celle de Howe [1974, prop. 5]; i.e., remplacer la fonction $\mu_{\alpha}^{1} \Psi_{\kappa}^{v}$ dans (A.7) par une fonction localement intégrable sur $\overline{\mathfrak{v}}_{\alpha}$, disons $\bar{b}_{\kappa}$, indépendante de $v$. Pour $\kappa=1$, la formule obtenue est très simple $\left(\bar{b}_{1}\right.$ est la mesure de Dirac en 0) et permet d'une part de montrer l'intégrabilité locale de la distribution $\Theta_{\alpha}^{\vee}=\left(\Theta_{\alpha}^{1}\right)^{\vee}$, d'autre part de calculer explicitement la fonction $\left.\Theta_{\alpha}^{\vee}\right|_{A^{\prime}}$ [Lemaire 2004, 1.8, 1.9]. Pour $\kappa \neq 1$, la situation est plus compliquée; en particulier, il semble difficile d'obtenir à partir d'une telle formule les renseignements escomptés sur la distribution $\left(\Theta_{\alpha}^{\kappa}\right)^{\vee}$ (resp. sur la fonction $\left.\left(\Theta_{\alpha}^{\kappa}\right)^{\vee}\right|_{A^{\prime}}$ ).

\section{Remerciement}

Je remercie Philippe Gille pour son aide dans la démonstration du lemme 5.3. 


\section{Références}

[Assem 1994] M. Assem, "The Fourier transform and some character formulae for $p$-adic $\mathrm{SL}_{l}, l$ a prime”, Amer. J. Math. 116 :6 (1994), 1433-1467. MR 96i :22037 Zbl 0837.20051

[Clozel 1987] L. Clozel, "Characters of nonconnected, reductive p-adic groups", Canad. J. Math. 39 :1 (1987), 149-167. MR 88i :22039 Zbl 0629.22008

[Hales 1993] T. C. Hales, "Unipotent representations and unipotent classes in SL(n)", Amer. J. Math. 115 :6 (1993), 1347-1383. MR 95a :22024 Zbl 0810.22008

[Harish-Chandra 1964] Harish-Chandra, "Invariant distributions on Lie algebras", Amer. J. Math. 86 (1964), 271-309. MR 28 \#5144 Zbl 0131.33302

[Harish-Chandra 1970] Harish-Chandra, Harmonic analysis on reductive p-adic groups, Lecture Notes in Math 162, Springer, Berlin, 1970. MR 54 \#2889 Zbl 0202.41101

[Harish-Chandra 1978] Harish-Chandra, "Admissible invariant distributions on reductive $p$-adic groups", pp. 281-347 dans Lie theories and their applications (Kingston, Ont., 1977), édité par W. Rossmann, Queen's Papers in Pure Appl. Math. 48, Queen's Univ., Kingston, Ont., 1978. MR 58 \#28313 Zbl 0433.22012

[Harish-Chandra 1980] Harish-Chandra, "A submersion principle and its applications", pp. 95-102 dans Geometry and analysis : Papers dedicated to the memory of V. K. Patodi, Indian Acad. Sci., Bangalore, 1980. MR 82e :22032 Zbl 0485.22023

[Henniart 1984] G. Henniart, La conjecture de Langlands locale pour GL(3), Mém. Soc. Math. France (N.S.) 11-12, Soc. math. de France, Paris, 1984. MR 86g :11070 Zbl 0564.12020

[Henniart 2001] G. Henniart, "Représentations des groupes réductifs $p$-adiques et de leurs sousgroupes distingués cocompacts", J. Algebra 236 :1 (2001), 236-245. MR 2001m :22036 Zbl 0980. 22019

[Henniart et Herb 1995] G. Henniart et R. Herb, "Automorphic induction for GL $(n)$ (over local non-Archimedean fields)”, Duke Math. J. 78 :1 (1995), 131-192. MR 96i :22038 Zbl 0849.11092

[Henniart et Lemaire 2004a] G. Henniart et B. Lemaire, "Existence de pseudo-coefficients pour les caractères tordus des séries $\kappa$-discrètes de $\operatorname{GL}(n, F)$ ", prépublication, 2004.

[Henniart et Lemaire 2004b] G. Henniart et B. Lemaire, "Intégrales orbitales tordues sur GL $(n, F)$ et corps locaux proches; applications", prépublication 2004-08, Université Paris-Sud 11 (Orsay), 2004, http ://www.math.u-psud.fr/ biblio/ppo/2004/ppo2004-08.html. À paraître dans Canad. J. Math.

[Howe 1974] R. Howe, “The Fourier transform and germs of characters (case of $\mathrm{Gl}_{n}$ over a $p$-adic field)”, Math. Ann. 208 (1974), 305-322. MR 49 \#7391 Zbl 0266.43007

[Huntsinger 1997] R. C. Huntsinger, Some aspects of invariant harmonic analysis on the Lie algebra of a reductive p-adic group, Ph.D. thesis, University of Chicago, 1997.

[Kazhdan 1984] D. Kazhdan, “On lifting”, pp. 209-249 dans Lie group representations (College Park, MD, 1982/1983), vol. II, édité par R. Herb et al., Lecture Notes in Math. 1041, Springer, Berlin, 1984. MR 86h :22029 Zbl 0538.20014

[Laumon 1996] G. Laumon, Cohomology of Drinfeld modular varieties, I : Geometry, counting of points and local harmonic analysis, Cambridge Studies in Advanced Mathematics 41, Cambridge University Press, Cambridge, 1996. MR 98c :11045a Zbl 0837.14018

[Lemaire 1997] B. Lemaire, Intégrales orbitales sur $\mathrm{GL}(N, F)$ où $F$ est un corps local non archimédien, Mém. Soc. Math. Fr. (N.S.) 70, Soc. math. de France, Paris, 1997. MR 99j :22016 Zbl 0914.22020 
[Lemaire 2004] B. Lemaire, "Intégrabilité locale des caractères tordus de $\mathrm{GL}_{n}(D)$ ", J. Reine Angew. Math. 566 (2004), 1-39. MR 2005h :22028 Zbl 1035.22012

[Rudin 1962] W. Rudin, Fourier analysis on groups, Tracts in Pure and Applied Mathematics 12, Wiley-Interscience, New York, 1962. MR 27 \#2808 Zbl 0107.09603

Received November 18, 2003. Revised November 2, 2004.

BERTRAND LEMAIRE

CNRS (UMR 8628)

UNIVERSITÉ DE PARIS-SUd

MATHÉMATIQUES, BÂT. 425

91405 ORSAY CEDEX

FRANCE

Bertrand.Lemaire@math.u-psud.fr 